\title{
Vascular ventures
}

Citation for published version (APA):

Haeren, RHL. (2018). Vascular ventures: Analysis of vascular structures and function in epilepsy. [Doctoral Thesis, Maastricht University]. Datawyse / Universitaire Pers Maastricht. https://doi.org/10.26481/dis.20181026rh

Document status and date:

Published: 01/01/2018

DOI:

10.26481/dis.20181026rh

Document Version:

Publisher's PDF, also known as Version of record

\section{Please check the document version of this publication:}

- A submitted manuscript is the version of the article upon submission and before peer-review. There can be important differences between the submitted version and the official published version of record.

People interested in the research are advised to contact the author for the final version of the publication, or visit the DOI to the publisher's website.

- The final author version and the galley proof are versions of the publication after peer review.

- The final published version features the final layout of the paper including the volume, issue and page numbers.

Link to publication

\footnotetext{
General rights rights.

- You may freely distribute the URL identifying the publication in the public portal. please follow below link for the End User Agreement:

www.umlib.nl/taverne-license

Take down policy

If you believe that this document breaches copyright please contact us at:

repository@maastrichtuniversity.nl

providing details and we will investigate your claim.
}

Copyright and moral rights for the publications made accessible in the public portal are retained by the authors and/or other copyright owners and it is a condition of accessing publications that users recognise and abide by the legal requirements associated with these

- Users may download and print one copy of any publication from the public portal for the purpose of private study or research.

- You may not further distribute the material or use it for any profit-making activity or commercial gain

If the publication is distributed under the terms of Article $25 \mathrm{fa}$ of the Dutch Copyright Act, indicated by the "Taverne" license above, 
(C) Roel Haeren, Sittard, 2018

No part of this book may be reproduced or transmitted in any form or by any means, without prior permission in writing by the author, or when appropriate, by the publishers of the publications.

Layout: Tiny Wouters

Cover design: "Brain cells" by Juan Gaertner

Production: Datawyse | Universitaire Pers Maastricht

ISBN: 9789493019577

The research described in this thesis was conducted at the department of Neurosurgery of the Maastricht University Medical Center, the School for Mental Health and Neurosciences and the Cardiovascular Research Institute Maastricht of the University of Maastricht, located in Maastricht, the Netherlands.

This research received no specific grant from any funding agency in the public, commercial or not-for-profit sectors. 


\title{
Vascular ventures
}

\section{Analysis of vascular structures and function in epilepsy}

\author{
PROEFSCHRIFT \\ ter verkrijging van de graad van doctor aan de Universiteit Maastricht, \\ op gezag van de Rector Magnificus, Prof. dr. Rianne M. Letschert \\ volgens het besluit van het College van Decanen, \\ in het openbaar te verdedigen op vrijdag 26 oktober 2018, om 12.00 uur \\ door
}

Roel Hubert Louis Haeren 


\section{Promotor}

Prof. dr. Y. Temel

\section{Co-promotores}

Dr. K. Rijkers

Dr. G. Hoogland

\section{Beoordelingscommissie}

Prof. dr. R.J. van Oostenbrugge (voorzitter)

Prof. dr. ir. W. Backus

Dr. A. Colon, Academisch Centrum voor Epileptologie, Kempenhaeghe, Heeze

Prof. dr. P. Fabene, University of Verona, Verona, Italy

Dr. S. Reitsma, Academisch Medisch Centrum Amsterdam 
Voor mijn lieve ouders 



\section{Contents}

Chapter 1 General introduction 9

Chapter 2 Assessment and imaging of the cerebrovascular glycocalyx: 25

a literature study

Chapter 3 Protocol for intraoperative assessment of the human

cerebrovascular glycocalyx

Chapter 4 In vivo imaging of the human cerebral microcirculation and its glycocalyx: a feasibility study

Chapter 5 Cerebral artery vasoconstriction requires neurogenic and adrenergic interaction and is endothelin-1 dependent

Chapter 6 Lipofuscin in the cerebral vascular wall: a technical and explorative analysis

Chapter 7 General discussion and conclusion

Summary

Samenvatting

Valorisation

Dankwoord

Curriculum vitae 



\section{Chapter 1}

General introduction 



\section{General introduction}

\section{Background}

In recent years, novel insights have created a paradigm shift in the study on the pathophysiology of neurological disorders. The traditional pathophysiological concepts centered around the neuron have made place for a more integrative model emphasizing interactions between different cell types within the brain ${ }^{1}$. In particular, cellular interactions at the cerebrovascular interface have gained increasing attention $^{2-6}$.

This paradigm shift is certainly taking place in epilepsy research. After many years of basic and clinical research centered around the neuron, the pathophysiology of epilepsy has not been fully elucidated. Currently, around $25 \%$ of epilepsy patients is not responding to medical treatment. These patients are awaiting new treatment options, that may be aimed at yet unraveled mechanisms. Interactions between neurons and vascular cells have been implicated to play a pivotal role in the pathophysiology of epilepsy and hence are potential targets for novel antiepileptic therapies ${ }^{7-9}$.

This thesis describes research exploring neurovascular interactions by studying structural and functional properties of the cerebral vasculature in relation to normal physiology of the brain and the pathophysiology of epilepsy. This introduction commences by describing the need for novel antiepileptic therapies, followed by an introduction on the significance of cerebral vasculature structure and function with respect to brain physiology. Next, a summary of clinical and experimental data is presented regarding structural and functional vascular abnormalities in epilepsy. This introduction then finishes by outlining the main topics, hypotheses, and research questions that are addressed in this thesis.

\section{Epilepsy}

According to the World Health Organization (WHO) epilepsy is a chronic neurological disorder characterized by recurrent unprovoked seizures, or a diagnosis of an epilepsy syndrome based on syndrome specific characteristics ${ }^{10}$. Seizures are brief episodes of involuntary movements of a part of the body (focal) or the entire body (generalized), that can be accompanied by loss of consciousness, bladder control, and bowel function $^{10}$. Seizures are the clinical expression of sudden, excessive electrical discharges in a cluster of cerebral neurons. The incidence and prevalence of epilepsy has been estimated to be 25-50 per 100.000 and 4-10 per 1.000, respectively ${ }^{11,12}$.

Epilepsy can be classified by semiology, seizure type (focal or generalized), epilepsy syndrome, and etiology ${ }^{13}$. The most prevalent type of focal epilepsy (seizures start in a 
specific part of the brain) is temporal lobe epilepsy (TLE). TLE encompasses $21-24 \%$ of epilepsy syndromes ${ }^{11}$. In this thesis, we have mainly studied patients suffering from TLE.

\subsection{Treatment of epilepsy}

Epilepsy is primarily treated by anti-epileptic drugs (AEDs), aimed at achieving seizure freedom with minimal side-effects. Between $20-40 \%$ of TLE-patients are drugresistant ${ }^{13,14}$. This means that in these patients adequate trials of two or more welltolerated and appropriately chosen, used and dosed AEDs scheduled to achieve sustained seizure freedom have failed ${ }^{15}$.

Drug-resistant TLE-patients can alternatively be treated by resective brain surgery. Outcome of surgery strongly depends on thorough preoperative evaluation to ascertain seizure focus. Surgery generally includes resection of the anterior pole of the temporal lobe with or without resection of the amygdala and hippocampus (anterior temporal lobectomy, ATL). Seizure freedom following ATL is reported in $65-73 \%$ of cases after 2 years, and in $62 \%$ at 12 -year follow-up, and quality of life increases ${ }^{14,16-22}$. Surgeryrelated complications include visual field deficits, cognitive complaints, neurological deficits like hemiparesis and dysphasia, and infections ${ }^{23}$. Other surgical options in extratemporal or multifocal epilepsy include tailored focus resection, vagus nerve stimulation, and deep brain stimulation, all leading to reduction in seizure frequency, yet results are lagging when compared to resective surgery.

Within the Maastricht University Medical Center + (MUMC+), the department of neurosurgery strongly focuses on epilepsy surgery. The MUMC+ is one of three university hospitals performing epilepsy surgery in The Netherlands. Together with the departments of neurology, radiology, neurophysiology, and epilepsy center Kempenhaeghe, the Academic Center for Epileptology was founded in 2014. This multidisciplinary cooperation results in a high number of epilepsy patients undergoing various types of epilepsy surgery, including resective surgery, vagus nerve stimulation, and deep brain stimulation. In the MUMC+, thirty to forty resective procedure are performed yearly.

\subsection{Impact of drug-resistant epilepsy}

Despite the availability of the above-mentioned treatment options, many patients remain therapy refractory. The societal burden of chronic therapy-resistant epilepsy is enormous and encompasses around $80 \%$ of total epilepsy-related costs ${ }^{24}$. Direct medical costs include AEDs, seizure-related injuries, and chronic follow-up by a neurologist. Indirect medical and societal costs are attributed to epilepsy-associated decreased social functioning related to psychiatric morbidities, such as cognitive decline, depression and anxiety, and illustrated by lower employment rates, high rates of sick leave, and driver's license restrictions ${ }^{25,26}$. Not surprisingly, self-reported quality of life is low in these patients ${ }^{27}$. 


\section{Structure and function of the cerebral vasculature}

The brain is the most vascularized organ of the human body, receiving around $20 \%$ of total blood flow. The cerebral vasculature consists of supplying arteries and arterioles, an extensive capillary bed, and effluent parenchymal venules and sinuses. The cerebral vasculature supplies brain tissue with oxygen and nutrients and drains tissue waste products, thereby contributing to homeostasis and consequently preserving physiological neuronal functioning. Brain tissue is highly dependent on adequate vascular function due to the lack of energy reserves. Because of the continuously changing activity of brain regions, and their corresponding metabolic rates, the cerebral vasculature is provided with several mechanisms to adapt local blood flow and to optimize microvascular conditions for exchange. These mechanisms are mainly based on interactions between brain and vascular tissue, and are performed at three levels: capillaries, parenchymal arterioles and pial arteries. Structural and functional characteristics of these vascular levels are discussed below and are summarized in Table 1.1 and Figure 1.1.

\begin{tabular}{|l|l|}
\hline Neurovascular unit & Definitions \\
\hline Neurovascular coupling & $\begin{array}{l}\text { A system at the capillary and arteriolar level, in which neurons, glial } \\
\text { (astrocytes, microglia) and vascular cells (endothelium cells, pericytes, } \\
\text { smooth muscle cells) co-operate by intercellular signaling to optimize } \\
\text { conditions for blood - brain exchange. }\end{array}$ \\
\hline $\begin{array}{l}\text { A mechanism that controls blood flow in order to meet local metabolic } \\
\text { demands of brain tissue. This mechanism is executed by the neurovascular } \\
\text { unit, i.e. metabolic demands are signaled from the brain tissue by neuronal } \\
\text { and glial cells towards the vascular endothelium, pericytes and smooth } \\
\text { muscle cells of capillaries and parenchymal arterioles. }\end{array}$ \\
\hline Vascular tone & $\begin{array}{l}\text { A mechanism that aims to regulate cerebral blood flow within a range } \\
\text { independent of arterial pressure alterations. This mechanism mainly involves } \\
\text { myogenic responses within pial arteries. }\end{array}$ \\
\hline Vasomotor control & $\begin{array}{l}\text { Vascular tone describes the degree of contraction of smooth muscle cells } \\
\text { within arteries and arterioles and thereby influences vessel diameter. }\end{array}$ \\
\hline $\begin{array}{l}\text { A mechanism that controls smooth muscle cell contraction. This control } \\
\text { mechanism is the result of neuronal (perivascular nerves, cerebral neurons } \\
\text { and glia cells), metabolic (neurovascular coupling), endothelial, and direct } \\
\text { myogenic (stretch-induced) signals. }\end{array}$ \\
\hline
\end{tabular}

Table 1.1 Summary of physiological vascular concepts and mechanisms that are introduced in this paragraph.

\subsection{Cerebral capillaries and permeability}

Starting downstream, cerebral capillaries consist of endothelial cells, that are interconnected by tight and adherens junctions ${ }^{3}$. These endothelial cells are surrounded by a basal lamina, pericytes, and astrocytic endfeet, that form a stabilizing network ${ }^{3}$. Together with surrounding neurons and microglia, these cells constitute the blood-brain barrier (BBB). Capillaries' most prominent function involves exchange of 
vital nutrients and waste products between blood plasma and brain tissue ${ }^{3,28}$. Transport of plasma components to the brain tissue is actively regulated by the cerebral tissue, thereby maintaining homeostasis ${ }^{3}$. Endothelial cells are considered key players in determining capillary permeability within the $\mathrm{BBB}^{3}$.

Current structural and functional concepts of the cerebral capillaries do not include the endothelial glycocalyx. The glycocalyx consists of glycoproteins and proteoglycans that form a gel-like matrix lining the luminal side of the endothelium ${ }^{29}$. The glycocalyx is involved in many endothelial functions, like leukocyte trafficking, and endothelial remodeling in response to shear stress ${ }^{30,31}$. Since the glycocalyx forms a physical barrier between the endothelium and plasma components, its condition has also been related to barrier properties of the endothelium in capillary beds ${ }^{30,32,33}$. Hence, the glycocalyx could also play a role in cerebral capillary permeability. The glycocalyx is assumed to attach to the endothelium in all types of vessels throughout the human body. The existence and properties of the cerebrovascular glycocalyx have however been assessed sparsely ex vivo, and not at all in vivo, nor in relation to neurological disorders ${ }^{34,35}$

\subsection{Parenchymal arterioles and neurovascular coupling}

Upstream of the capillary bed, parenchymal arterioles are characterized by endothelial cells that are surrounded by a few layers of smooth muscle cells ${ }^{6,36}$. These smooth muscle cells are completely covered by astrocytic endfeet ${ }^{6}$. Parenchymal arterioles are mainly concerned with adapting local cerebral blood flow. Adaptation of cerebral blood flow is regulated to meet continuously changing metabolic demands of downstream brain tissue ${ }^{6,37,38}$. This process is called neurovascular coupling and is performed within the neurovascular unit ${ }^{6,39}$.

The neurovascular unit is composed of vascular, neuronal, and glial cells at both the capillary and arteriolar level. These cells co-operate as a unit to regulate cerebral blood flow $^{39,40}$. Metabolic demands from brain tissue are continuously signaled by neuronal and glial cells towards the vascular cells of parenchymal arterioles. These arterioles respond by increasing or reducing blood flow by means of vasodilatation or vasoconstriction, respectively. The neurovascular unit also aims to optimize conditions for exchange of oxygen, nutrients, and waste products between the blood and brain tissue. By example, local tissues can cope with chronic high metabolic demands by increasing the proportion of perfused capillaries, i.e. capillary recruitment, thereby optimizing exchange conditions ${ }^{41}$. 


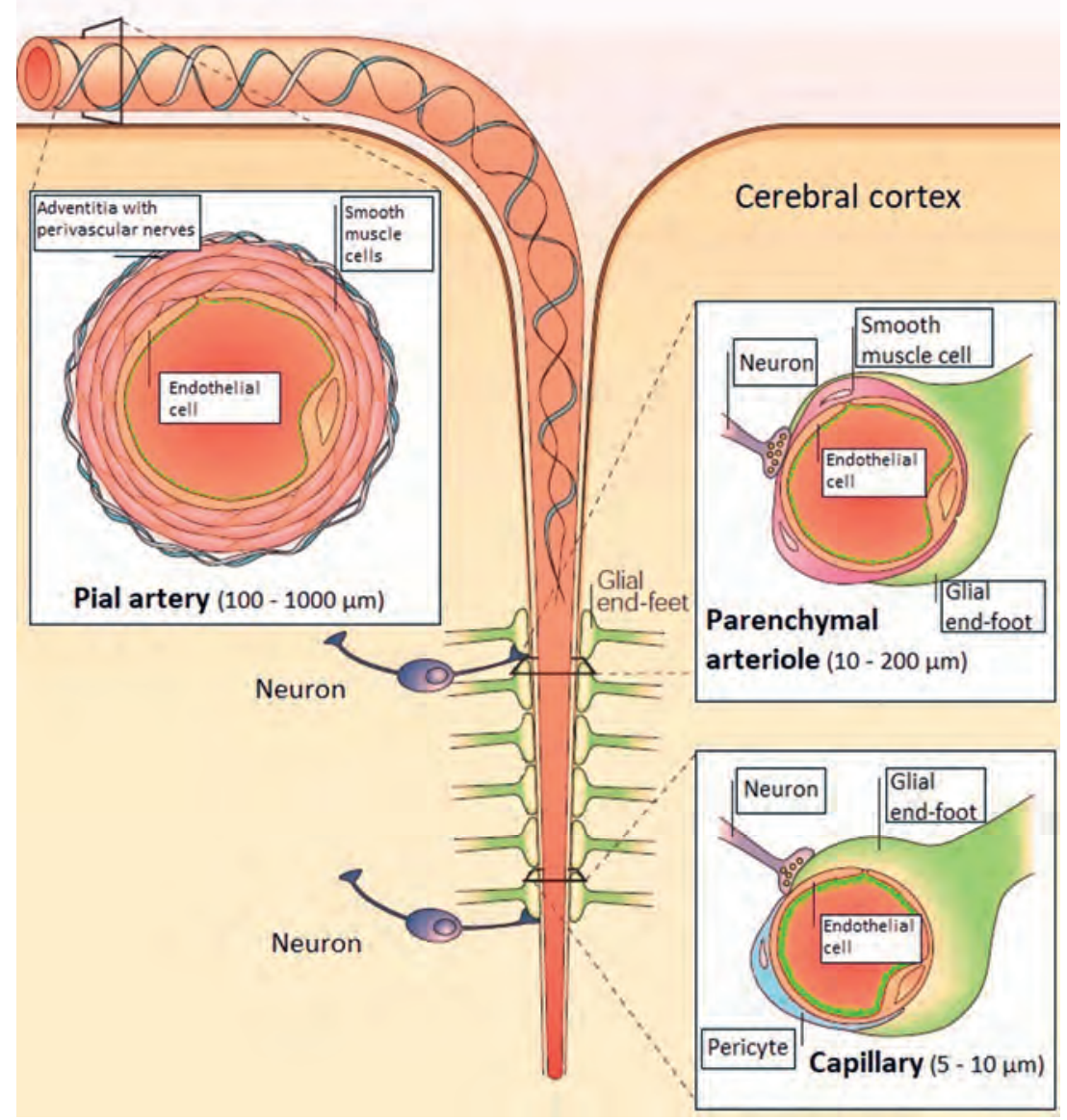

Figure 1.1 Figure 1 (adapted from ladecola et al. 2004, with permission): the cerebral vasculature is characterized by three levels that differ by structure and function:

I. Capillaries are formed by endothelial cells and the endothelial glycocalyx (green layer) that aligns the luminal side of endothelial cells. The endothelium is surrounded by pericytes within the basal lamina, astrocyte (glial) end-feet, and neurons. Together these cells constitute the blood-brain barrier and provide the surface for blood-brain exchange of vital nutrients and waste products. Also, capillaries are involved in neurovascular coupling by signaling metabolic alterations towards upstream parenchymal arterioles.

II. Parenchymal arterioles consist of endothelial cells, including the glycocalyx (green layer), covered by one to three layers of smooth muscle cells that themselves are in direct contact with astrocytic end-feet and neurons. Their main function is adaptation of blood flow to the downstream capillaries that is mainly regulated by neurovascular coupling. Neurovascular coupling signals come from the downstream capillaries and the surrounding glial cells and neurons.

III. Pial arteries are characterized by endothelial cells, including the glycocalyx (green layer), that are surrounded by multiple smooth muscle cell layers. The outer layer of pial arteries is called 'adventitia' and contains perivascular neurons involved in adaptation of vascular tone. Pial arteries are mainly concerned with autoregulation of local cerebral blood flow. In addition, pial arteries determine local blood flow based on endothelial-, neuronal-, and metabolicderived input altering vascular tone. 


\subsection{Pial arteries and autoregulation}

Further upstream, pial arteries consist of a luminal endothelial cell layer surrounded by multiple smooth muscle cell layers. These smooth muscle cells are covered by the adventitia containing leptomeningeal cells including perivascular nerves ${ }^{6,36}$. Pial arteries are responsible for most of cerebrovascular autoregulation. This autoregulation mechanism controls cerebral blood flow by changing vascular tone within a specific range, independent of changes in arterial pressure ${ }^{42}$. Autoregulation is mainly a myogenic response that is regulated by changes in luminal hydrostatic pressure causing stretch-induced modulation of smooth muscle cells contractility ${ }^{43}$. Autoregulation limits the damaging effects of hyper- or hypo-perfusion and supports distribution of blood flow according to brain metabolic needs. Autoregulation and neurovascular coupling cause changes in the vascular tone of pial arteries and parenchymal arterioles. Vascular tone is predominantly determined by contraction and relaxation of arteriolar smooth muscle cells. The underlying mechanisms regulating vascular tone, such as myogenic responses, are described as vasomotor control.

Other mechanisms involved in adjusting vasomotor control of cerebral arteries include metabolic, neurogenic, and endothelial responses ${ }^{36,44-46}$. The metabolic mechanisms are mainly based on neurovascular coupling, as described above. The neurogenic mechanisms are exerted by adventitial perivascular nerves releasing neurotransmitters. Classically, sympathetic adrenergic perivascular innervation, by releasing noradrenalin, is held responsible for most of the vasoconstrictor tone ${ }^{47-50}$. However, the precise influence of sympathetic nerves and their neurotransmitter noradrenalin in cerebral arteries is still debated. Some reported strong contractile responses to stimulation of perivascular sympathetic nerves, whereas others found no response at all ${ }^{51-58}$. Finally, the endothelial mechanisms include the stretch- and shear stress-induced release of vasoactive substances like nitric oxide and endothelin $-1^{44,59}$. Recent evidence suggests that endothelin-1 plays an important role in vasoconstriction of peripheral and cerebral $\operatorname{arteries}^{50,60,61}$.

\section{Role of vascular abnormalities in the pathophysiology of epilepsy}

The reported long-term risk of developing epilepsy following a cerebrovascular accident is $2-15 \%^{62,63}$. Seizure incidence is six to ten times higher in patients suffering from neurodegenerative disorders than in the general population ${ }^{64}$. Conversely, epilepsy patients are two to five times more likely to suffer from stroke than the general population $^{65}$. Based on this bidirectional relation between stroke and epilepsy, Brigo et al. recently suggested that epilepsy might be the first symptom of underlying occult cerebrovascular pathology ${ }^{66}$. Various vascular abnormalities have indeed been reported 
in TLE-patients. These include structural abnormalities on the one hand, and impaired function of the cerebral microcirculation on the other ${ }^{8,67,68}$.

\subsection{Structural vascular abnormalities}

In the sclerotic hippocampus of TLE-patients increased vessel density has been observed ex vivo ${ }^{68-71}$. Contradictory, Kastanauskaite et al. reported on a reduced density of normal microvessels ${ }^{72}$. They concluded that the increase reported by others was due to non-functional, atrophic, and tortuous vascular structures, that were erroneously taken into account during ex vivo imaging ${ }^{72}$. These contradicting reports invite to address the cerebral microcirculation of these patients by applying various techniques including analysis of the functional microcirculation that is only available when assessed in vivo.

\subsection{Capillary abnormalities in epilepsy}

Abnormalities in capillary structure include reduced numbers of tight junctions, increased basal lamina thickness, and altered endothelial - basal lamina connectivity, as reported in preclinical epilepsy models and epilepsy patients ${ }^{8,9,69}$. Functionally, reduced integrity of cerebral capillaries may result in increased permeability of the BBB and loss of capillaries. Indeed, extravasation of serum leucocytes, albumin and IgG into the parenchymal tissue has been described in relation to the pathophysiology of epilepsy ${ }^{7,37,73,74}$. This extravasation can result in altered astrocytic function, upregulation of proinflammatory cytokines, enhanced neuronal excitability, and impaired brain homeostasis ${ }^{8,73,75}$.

As suggested above, the glycocalyx should be considered part of the endothelial barrier of cerebral capillaries. An intact glycocalyx may contribute to the physiological prevention of albumin and leucocyte extravasation. A disrupted glycocalyx on the other hand may initiate the above described pathophysiological processes. Both animal and human studies have shown that glycocalyx disruption in other organs results in inflammation, oxidative stress, and an altered vascular responsiveness ${ }^{76}$. These pathological mechanisms have also been implicated in the pathophysiology epilepsy ${ }^{8,73,75}$.

In this regard, disruption of the cerebrovascular glycocalyx could be related to the development of epilepsy in patients. Thus, assessment of the cerebrovascular glycocalyx in epilepsy patients could be an interesting research topic. To the best of our knowledge, the cerebrovascular glycocalyx has not been studied in clinical nor preclinical epilepsy research.

\subsection{Dysfunctional autoregulation and neurovascular coupling}

During seizures, blood pressure and heart rate acutely rise to sometimes extreme peaks $^{42}$. Consequently, autoregulatory mechanisms of pial arteries are repetitively 
challenged in patients suffering from chronic epilepsy ${ }^{77}$. In addition, sudden and repetitive increases in metabolism during seizures challenges the regulation of cerebral blood flow. Moreover, adequate neurovascular coupling and control of vasomotor tone of cerebral arteries are likely to be disturbed by remodeled capillary beds, leaky microvessels, and local inflammation ${ }^{8,75}$. This emphasizes the importance to further analyze the mechanisms of vasomotor control, like adrenergic mechanisms in vasoconstriction, that are involved in autoregulation.

\subsection{Vascular dysfunction may result in oxidative stress}

Due to epilepsy-associated neurovascular changes, neuronal and vascular cells of the neurovascular unit are continuously challenged. During a seizure, the challenge on these vascular cells is further aggravated by hemodynamic changes and a metabolically hyperactive brain, which might lead to oxidative stress ${ }^{37}$. Oxidative stress applies to a condition of high cellular metabolic activity leading to excessive reactive oxygen species generation that cannot be counterbalanced by the antioxidant defense system resulting in structural cell damage ${ }^{78-80}$. A reciprocal relation between oxidative stress and microvascular dysfunction has been described ${ }^{4,81,82}$. Moreover, seizure-induced oxidative stress in the brain of epilepsy patients has been suggested ${ }^{83}$. This may affect brain function and neurovascular interaction, thereby contributing to the concept of 'accelerated ageing' covering many aspects of epilepsy including cognitive decline $e^{4,26,84}$.

\section{Delineating the research questions of this thesis}

Based on the aforementioned concepts, we conclude that structural and functional cerebrovascular abnormalities are present in TLE-patients. In this thesis, the possibilities to assess cerebrovascular structures and their function during brain surgery and in surgically resected brain tissue are explored. Topic selection was based on the unique combination of available surgical and scientific experience in the epilepsy surgery team, and the vascular scientific experience available in research school CARIM, that has a long tradition in vascular research. For our epilepsy research team, this was the first acquaintance with vascular research. Hence, the nature of the performed experiments is explorative as well as innovative. We have selected three vascular topics to explore in this thesis: 1 . in vivo assessment of the cerebrovascular glycocalyx, 2. vasomotor control of cerebral artery vasoconstriction, and 3. arterial structure. 


\section{Hypothesis I}

The cerebrovascular glycocalyx is disrupted in temporal lobe epilepsy patients

This hypothesis is based on the assumption that the glycocalyx is lining the endothelium of cerebral capillaries. Since the cerebrovascular glycocalyx has only rarely been studied to date, the first step was to review which techniques are available to assess the glycocalyx. Therefore, the first research question of this thesis was:

\section{What are the available techniques to assess the cerebrovascular glycocalyx?}

In our neurosurgical department, resective epilepsy surgery as well as several other types of brain surgery are performed. During these craniotomies, the cerebral microcirculation is literally exposed, and hence directly available for evaluation. It was therefore attempted to assess the cerebral microcirculation intraoperatively using a technique that allows real-time glycocalyx assessment as well. A literature study resulted in selecting side-stream dark field imaging as the most suitable technique for this assessment (chapter 2). Subsequently, a case-control study was designed to address our first hypothesis (chapter $\mathbf{3}$ ). As this was the first time that this device was used to estimate the human cerebrovascular glycocalyx in vivo, the protocol of this study is described in chapter 3. Including this protocol aims to increase the internal validity, facilitates its reproducibility, and is in accordance with current scientific standards of transparency. Using this protocol, we aim to answer the following research question:

\section{Can the cerebrovascular glycocalyx be assessed using side-stream dark field imaging during resective brain surgery?}

Chapter $\mathbf{4}$ is dedicated to the detailed description of the first measurements performed in epilepsy patients and is presented as a feasibility study. Evaluation of the cerebrovascular glycocalyx by side-stream dark field imaging also enabled analysis of other microvascular variables, like vessel density. As mentioned, the case-control study was designed to address the first hypothesis. Since patient inclusion and measurements are currently ongoing, this thesis does not describe outcome of the complete study.

\section{Hypothesis II}

Cerebral artery vasoconstriction is controlled by endothelin-1

With regard to the regulation of cerebral artery vasoconstriction, the precise influence of sympathetic nerves and noradrenaline is unclear. Therefore, sympathetic and neurogenic vasomotor mechanisms in vasoconstriction are assessed in cerebral arteries 
isolated from resected brain tissue of epilepsy patients. This resulted in the following research question:

\section{Is endothelin-1 required for cerebral artery vasoconstriction?}

This research question is addressed in chapter 5 by reporting on experiments in which adrenergic, neurogenic, and endothelin-receptor modulators were applied. These modulators were applied on cerebral arteries that were obtained from epilepsy patients who underwent resective surgery. Due to a lack of freshly obtained human brain tissue from non-epileptic controls, the question if cerebral artery vasoconstriction is altered in epilepsy could not be addressed. As a surrogate control, rat cerebral artery vasoconstriction is assessed using the same vasoactive modulators.

\section{Hypothesis III}

The vascular wall of cerebral arteries of epilepsy patients shows signs of oxidative stress

During two-photon laser scanning microscopy assessment of epilepsy patients' cerebral arteries, autofluorescent particles within the vascular wall were noted by serendipity. Because of the abundance of these particles, we hypothesized that these particles might be related to epilepsy-associated vascular pathology. The nature of these particles was studied, and their presence in cerebral arteries and tissue was assessed in epilepsy patients and non-epileptic controls in order to answer the final two research questions of this thesis:

\section{What is the nature of the autofluorescent particles noted in the vascular wall of epilepsy patients?}

\section{Do these particles in the vascular wall have different properties in epilepsy patients when compared to controls?}

Chapter 6 describes the spectrometric analysis that was performed to identify these particles as lipofuscin. In order to find differences between epilepsy patients and (postmortem) controls, a quantitative assessment of the particles in the vascular wall of cerebral arteries was included additionally. 


\section{References}

1. Guo S, Lo EH. Dysfunctional cell-cell signaling in the neurovascular unit as a paradigm for central nervous system disease. Stroke. 2009;40:S4-7.

2. Rosenberg GA. Neurological diseases in relation to the blood-brain barrier. J Cereb Blood Flow Metab. 2012;32:1139-1151.

3. Abbott NJ, Patabendige AAK, Dolman DEM, Yusof SR, Begley DJ. Structure and function of the bloodbrain barrier. Neurobiol Dis. 2010;37:13-25.

4. Brown WR, Thore CR. Review: Cerebral microvascular pathology in ageing and neurodegeneration. Neuropathol Appl Neurobiol. 2011;37:56-74.

5. Toth P, Tarantini S, Csiszar A, Ungvari Z. Functional vascular contributions to cognitive impairment and dementia: mechanisms and consequences of cerebral autoregulatory dysfunction, endothelial impairment, and neurovascular uncoupling in aging. Am J Physiol Hear Circ Physiol. 2017;312: H1-20.

6. Iadecola C. Neurovascular regulation in the normal brain and in Alzheimer's disease. Nat Rev Neurosci. 2004;5:347-60.

7. Kovács R, Heinemann U, Steinhäuser C. Mechanisms underlying blood-brain barrier dysfunction in brain pathology and epileptogenesis: role of astroglia. Epilepsia. 2012;53 Suppl 6:53-9.

8. Heinemann U, Kaufer D, Friedman A. Blood brain barrier dysfunction, TGFb signaling and astrocyre dysfunction in epilepsy. Glia. 2012;60:1251-7.

9. van Vliet EA, Aronica E, Gorter JA. Role of blood-brain barrier in temporal lobe epilepsy and pharmacoresistance. Neuroscience. 2014;277:455-73.

10. WHO | Epilepsy. WHO. World Health Organization; 2016;

11. Engel J, McDermott MP, Wiebe S, et al. Early surgical therapy for drug-resistant temporal lobe epilepsy: a randomized trial. JAMA. 2012;307:922-30.

12. Téllez-Zenteno JF, Hernández-Ronquillo L. A review of the epidemiology of temporal lobe epilepsy. Epilepsy Res Treat. 2012;2012:630853.

13. Bell G., Sander J. The epidemiology of epilepsy: the size of the problem. Seizure. 2001;10:306-16.

14. Berg AT, Berkovic SF, Brodie MJ, et al. Revised terminology and concepts for organization of seizures and epilepsies: report of the ILAE Commission on Classification and Terminology, 2005-2009. Epilepsia. 2010;51:676-85.

15. Kwan P, Arzimanoglou A, Berg AT, et al. Definition of drug resistant epilepsy: consensus proposal by the ad hoc Task Force of the ILAE Commission on Therapeutic Strategies. Epilepsia. 2010;51:1069-77.

16. Hemb M, Palmini A, Paglioli E, et al. An 18-year follow-up of seizure outcome after surgery for temporal lobe epilepsy and hippocampal sclerosis. J Neurol Neurosurg Psychiatry. 2013;84:800-5.

17. Schmidt D, Stavem K. Long-term seizure outcome of surgery versus no surgery for drug-resistant partial epilepsy: A review of controlled studies. Epilepsia. 2009;50:1301-9.

18. von Lehe M, Lutz M, Kral T, Schramm J, Elger CE, Clusmann H. Correlation of health-related quality of life after surgery for mesial temporal lobe epilepsy with two seizure outcome scales. Epilepsy Behav. 2006;9:73-82.

19. Picot M-C, Jaussent A, Neveu D, et al. Cost-effectiveness analysis of epilepsy surgery in a controlled cohort of adult patients with intractable partial epilepsy: A 5-year follow-up study. Epilepsia. 2016; 57(10):1669-79.

20. Sajobi TT, Fiest KM, Wiebe S. Changes in quality of life after epilepsy surgery: The role of reprioritization response shift. Epilepsia. 2014;55:1331-8.

21. Markand ON, Salanova V, Whelihan E, Emsley CL. Health-related quality of life outcome in medically refractory epilepsy treated with anterior temporal lobectomy. Epilepsia. 2000;41:749-59.

22. Lertlum S, Tuchinda L, Shoungshotti C, Ounpak P. Quality of life after successful epilepsy surgery : evaluation by occupational achievement and income acquisition. 2005;88:207-13.

23. Bjellvi J, Flink R, Rydenhag B, Malmgren K. Complications of epilepsy surgery in Sweden 1996-2010: a prospective, population-based study. J Neurosurg. 2015;122:519-25.

24. Begley CE, Famulari M, Annegers JF, et al. The cost of epilepsy in the United States: an estimate from population-based clinical and survey data. Epilepsia. 2000;41:342-51.

25. Allers K, Essue BM, Hackett ML, et al. The economic impact of epilepsy: a systematic review. BMC Neurol. 2015;15:245. 
26. Dabbs K, Becker $\mathrm{T}$, Jones J, Rutecki $\mathrm{P}$, Seidenberg $\mathrm{M}$, Hermann B. Brain structure and aging in chronic temporal lobe epilepsy. Epilepsia. 2012;53:1033-43.

27. Taft C, Sager Magnusson E, Ekstedt G, Malmgren K. Health-related quality of life, mood, and patient satisfaction after epilepsy surgery in Sweden--a prospective controlled observational study. Epilepsia. 2014;55:878-85.

28. Jacob M, Chappell D, Becker BF. Regulation of blood flow and volume exchange across the microcirculation. Crit Care 2016;1-13.

29. Reitsma S, Slaaf DW, Vink H, Van Zandvoort MAMJ, Oude Egbrink MGA. The endothelial glycocalyx: Composition, functions, and visualization. Pflugers Arch Eur J Physiol. 2007;454:345-59.

30. Ebong EE, Lopez-Quintero S V, Rizzo V, Spray DC, Tarbell JM. Shear-induced endothelial NOS activation and remodeling via heparan sulfate, glypican-1, and syndecan-1. Integr Biol. (Camb). 2014;6:338-47.

31. Constantinescu AA, Vink H, Spaan JE. Endothelial cell glycocalyx modulates immobilization of leukocytes at the endothelial surface. Arterioscler Thromb Vasc Biol. 2003;23:1541-7.

32. Tarbell JM, Simon SI, Curry F-RE. Mechanosensing at the Vascular Interface. Annu Rev Biomed Eng. 2014;16:505-32.

33. Kolářová $H$, Ambrůzová $B$, Svihálková Šindlerová L, Klinke A, Kubala L. Modulation of endothelial glycocalyx structure under inflammatory conditions. Mediators Inflamm.; 2014;2014:694312.

34. Neuhaus W, Germann B, Plattner VE, Gabor F, Wirth M, Noe CR. Alteration of the glycocalyx of two blood-brain barrier mimicking cell lines is inducible by glioma conditioned media. Brain Res. 2009;1279:82-9.

35. Mazzetti S, Librizzi L, Frigerio S, de Curtis M, Vitellaro-Zuccarello L. Molecular anatomy of the cerebral microvessels in the isolated guinea-pig brain. Brain Res. 2004;999:81-90.

36. Hamel E. Perivascular nerves and the regulation of cerebrovascular tone. J Appl Physiol. 2006;100: 1059-64.

37. Marchi N, Lerner-Natoli M. Cerebrovascular remodeling and epilepsy. Neurosci. 2013;19:304-12.

38. Longden TA, Nelson MT. Vascular Inward Rectifier K+ Channels as External K+ Sensors in the Control of Cerebral Blood Flow. Microcirculation. 2016;22:183-96.

39. Muoio V, Persson PB, Sendeski MM. The neurovascular unit - concept review. Acta Physiol. 2014;210: 790-8.

40. Lok J, Gupta P, Guo S, et al. Cell-cell signaling in the neurovascular unit. Neurochem Res. 2007;32: 203245.

41. Tyagi A, Sethi AK, Girotra G, Mohta M. The microcirculation in sepsis. Indian J Anaesth. 2009 [cited 2017 Dec 25];53:281-93.

42. Iadecola C, Davisson RL. Hypertension and cerebrovascular dysfunction. Cell Metab. 2008;7(6):476-84.

43. Cipolla MJ. The Cerebral Circulation. San Rafael (CA): Morgan \& Claypool Life Sciences; 2009.

44. Dora KA. Coordination of vasomotor responses by the endothelium. Circ J. 2010;74:226-32.

45. Peterson EC, Wang Z, Britz G. Regulation of cerebral blood flow. Int J Vasc Med. 2011;2011:823525.

46. Andresen J, Shafi NI, Bryan RM. Endothelial influences on cerebrovascular tone. J Appl Physiol. 2006;100:318-27.

47. Nausch LWM, Bonev AD, Heppner TJ, Tallini Y, Kotlikoff MI, Nelson MT. Sympathetic nerve stimulation induces local endothelial Ca2+ signals to oppose vasoconstriction of mouse mesenteric arteries. AJP Hear Circ Physiol. 2012;302:H594-602.

48. Hilgers RHP, De Mey JGR. Myoendothelial coupling in the mesenteric arterial bed; segmental differences and interplay between nitric oxide and endothelin-1. Br J Pharmacol.2009;156:1239-47.

49. Van Riper DA, Bevan JA. Evidence that neuropeptide $Y$ and norepinephrine mediate electrical fieldstimulated vasoconstriction of rabbit middle cerebral artery. Circ Res. 1991;68:568-77.

50. Pagán RM, Martínez AC, Hernández M, et al. Endothelial and neural factors functionally involved in the modulation of noradrenergic vasoconstriction in healthy pig internal mammary artery. Biochem. Pharmacol. 2012;83:882-92.

51. Lee TJF, Chang HH, Lee HC, et al. Axo-axonal interaction in autonomic regulation of the cerebral circulation. Acta Physiol.2011;203:25-35.

52. Duckles SP, Bevan JA. Pharmacological characterization of adrenergic receptors of a rabbit cerebral artery in vitro. J Pharmacol Exp. 1976;197:371-8. 
53. Bevan RD, Dodge J, Nichols $\mathrm{P}$, et al. Weakness of sympathetic neural control of human pial compared with superficial temporal arteries reflects low innervation density and poor sympathetic responsiveness. Stroke. 1998;29:212-21.

54. Van Riper DA, Bevan JA. Selective variation of agonist and neurally mediated vasoconstriction with rabbit middle cerebral artery branch order. J Pharmacol Exp Ther.1991;257:879-86.

55. Handa Y, Caner H, Hayashi M, Tamamaki N, Nojyo Y. The distribution pattern of the sympathetic nerve fibers to the cerebral arterial system in rat as revealed by anterograde labeling with WGA-HRP. Exp Brain Res. 1990;82:493-8.

56. Westcott EB, Segal SS. Perivascular Innervation: A Multiplicity of Roles in Vasomotor Control and Myoendothelial Signaling. Microcirculation. 2013;20:217-38.

57. Aldasoro M, Martínez C, Vila JM, Medina P, Lluch S. Influence of endothelial nitric oxide on adrenergic contractile responses of human cerebral arteries. J Cereb Blood Flow Metab. 996;16:623-8.

58. Duckworth JW, Wellman GC, Walters CL, Bevan JA. Aminergic histofluorescence and contractile responses to transmural electrical field stimulation and norepinephrine of human middle cerebral arteries obtained promptly after death. Circ Res.1989;65:316-24.

59. Mccarron RM, Chen Y, Tomori T, et al. Endothelial-mediated regulation of cerebral microcirculation. J Physiol Pharmacol. 2006;57:133-44.

60. Thorin E, Nguyen TD, Bouthillier A. Control of vascular tone by endogenous endothelin-1 in human pial arteries. Stroke. 1998;29:175-80.

61. Compeer MG, Janssen GMJ, De Mey JGR. Endothelin-1 and endothelin-2 initiate and maintain contractile responses by different mechanisms in rat mesenteric and cerebral arteries. Br J Pharmacol. 2013;170:1199-209.

62. Bentes C, Martins H, Peralta AR, et al. Post-stroke seizures are clinically underestimated. J. Neurol. 2017;264(9):1978-1985

63. Zelano J. Poststroke epilepsy: update and future directions. Ther Adv Neurol Disord. 2016;9:424-35.

64. Beagle AJ, Darwish SM, Ranasinghe KG, La AL, Karageorgiou E, Vossel KA. Relative incidence of seizures and myoclonus in Alzheimer's disease, dementia with lewy bodies, and frontotemporal dementia. Cretin B, editor. J. Alzheimer's Dis. 2017;1-13.

65. Jin J, Chen R, Xiao Z. Post-epilepsy stroke: A review. Expert Rev Neurother. 2016;16:341-9.

66. Brigo F, Nardone R. Late-onset seizures: a subclinical cerebrovascular disorder? Expert Rev Neurother. 2017;17:751-3.

67. Thom M. Review: Hippocampal sclerosis in epilepsy: A neuropathology review. Neuropathol Appl Neurobiol. 2014:520-43.

68. Mott RT, Thore CR, Moody DM, Glazier SS, Ellis TL, Brown WR. Reduced ratio of afferent to total vascular density in mesial temporal sclerosis. J Neuropathol Exp Neurol. 2009;68:1147-54.

69. Rigau V, Morin M, Rousset M-C, et al. Angiogenesis is associated with blood-brain barrier permeability in temporal lobe epilepsy. Brain 2007;130:1942-56.

70. Morin-Brureau M, Lebrun A, Rousset M-C, et al. Epileptiform activity induces vascular remodeling and zonula occludens 1 downregulation in organotypic hippocampal cultures: role of VEGF signaling pathways. J Neurosci. 2011;31:10677-88.

71. Alonso-Nanclares L, DeFelipe J. Alterations of the microvascular network in the sclerotic hippocampus of patients with temporal lobe epilepsy. Epilepsy Behav. 2014;38:48-52.

72. Kastanauskaite A, Alonso-Nanclares L, Blazquez-Llorca L, Pastor J, Sola RG, DeFelipe J. Alterations of the microvascular network in sclerotic hippocampi from patients with epilepsy. J Neuropathol Exp Neurol. 2009;68:939-50.

73. van Vliet EA, Aronica E, Gorter JA. Blood-brain barrier dysfunction, seizures and epilepsy. Semin Cell Dev Biol. 2015;38:26-34.

74. Librizzi L, de Cutis M, Janigro D, et al. Cerebrovascular heterogeneity and neuronal excitability. Neurosci Lett Neurosci Lett. 2018;667:75-83

75. Vezzani A, Friedman A, Dingledine RJ. The role of inflammation in epileptogenesis. Neuropharmacology. 2013:16-24.

75. Vezzani A, Friedman A, Dingledine RJ. The role of inflammation in epileptogenesis. Neuropharmacology. 2013:16-24.

76. Dane MJC, van den Berg BM, Lee DH, et al. A microscopic view on the renal endothelial glycocalyx. Am J Physiol Renal Physiol. 2015;308(9):F956-66. 
77. Devinsky O. Effects of Seizures on Autonomic and Cardiovascular Function. Epilepsy Curr. 2004;4:43-6.

78. Czerska M, Mikołajewska K, Zieliński M, Gromadzińska J, Wąsowicz W. Today's oxidative stress markers. Med Pr. 2015;66:393-405.

79. Lushchak VI. Free radicals, reactive oxygen species, oxidative stress and its classification. Chem Biol Interact.; 2014;224:164-75.

80. Sies H. Oxidative stress: oxidants and antioxidants. Exp Physiol. 1997;82:291-5.

81. Najjar S, Pearlman DM, Devinsky O, Najjar A, Zagzag D. Neurovascular unit dysfunction with blood-brain barrier hyperpermeability contributes to major depressive disorder: a review of clinical and experimental evidence. J Neuroinflammation. 2013;10:142.

82. Islam MT. Oxidative stress and mitochondrial dysfunction-linked neurodegenerative disorders. Neurol. Res. 2017;39:73-82.

83. Menon B, Ramalingam K, Kumar RV. Oxidative stress in patients with epilepsy is independent of antiepileptic drugs. Seizure. 2012;21:780-4.

84. Schipper S, Aalbers MW, Rijkers K, et al. Accelerated cognitive decline in a rodent model for temporal lobe epilepsy. Epilepsy Behav. 2016;65:33-41. 


\section{Chapter

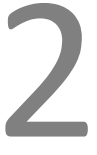

\section{Assessment and imaging of the cerebrovascular glycocalyx: a literature study}

R.H.L. Haeren, S.E.M. van de Ven, M.A.M.J. van Zandvoort, H. Vink, J.J. van Overbeeke, G. Hoogland, K. Rijkers Adapted from the publication in Current Neurovascular Research, 2016 


\section{Abstract}

The glycocalyx is a gel-like layer lining the luminal surface of the endothelium. The glycocalyx exerts an important barrier role because it prevents exposure of plasma components to the endothelial surface. Disruption of the glycocalyx by local inflammation or ischemia results in decreased glycocalyx thickness which is associated with a number of vascular diseases. The cerebrovascular glycocalyx has sparsely been studied but is of great interest because of its potential role in cerebrovascular diseases. In this literature study, we describe all existing techniques to visualize the glycocalyx and designate techniques that may be suitable for studying the cerebrovascular glycocalyx.

A total of seven imaging techniques are discussed thoroughly, including transmission electron microscopy, intravital microscopy, micro-particle image velocimetry, confocal laser scanning microscopy, two-photon laser scanning microscopy, orthogonal polarization spectral imaging and sidestream dark field/oblique imaging. Measurement of serum concentrations of glycocalyxspecific constituents is another method for glycocalyx analysis. Also, we have reviewed the methods of glycocalyx analysis by using these imaging techniques.

So far, the cerebrovascular glycocalyx has only been studied in vitro. However, other cerebral microcirculatory properties have been studied in vivo. This suggests that the cerebrovascular glycocalyx can be studied in vivo by using some of the described techniques, when specific software is subjoined to the analysis.

In conclusion, we have summarized techniques available for glycocalyx assessment, and explained the significance and technical possibilities regarding cerebrovascular glycocalyx visualization. Cerebrovascular glycocalyx assessment would add valuable information to our understanding of the pathophysiology of cerebrovascular disease. Moreover, as a part of the blood-brain barrier, more knowledge on the cerebrovascular glycocalyx may lead to better understanding of neurodegenerative conditions that are caused by a compromised blood-brain barrier including Alzheimer's disease, vascular dementia, multiple sclerosis and epilepsy. 


\section{Introduction}

Adequate flow of blood through the arterioles and capillaries - the microcirculation - is essential for tissue nutritional exchange and oxygen supply. Structural and functional changes of the microcirculation are associated with diseases such as hypertension, sepsis and diabetes mellitus ${ }^{1-3}$. One of the most recently identified microvascular structures is the endothelial glycocalyx, which we will further refer to as 'glycocalyx'. Imaging of microcirculatory structures, including the glycocalyx, has been performed in order to understand the pathophysiology of several medical conditions. However, the cerebrovascular glycocalyx has hardly been studied. Nevertheless, it is of great scientific and clinical interest because of its potential role in cerebrovascular disease and, as a potential part of the blood-brain barrier, in the pathophysiology of neurological diseases such as Alzheimer's disease, vascular dementia, multiple sclerosis and epilepsy. ${ }^{4-10}$ In this review, we describe the techniques that are currently available for visualization of the glycocalyx. Also, we have reviewed the methods of glycocalyx analysis by using these imaging techniques and we discuss whether these techniques are suitable to assess the cerebrovascular glycocalyx.

\subsection{The glycocalyx}

The glycocalyx is a gel-like layer lining the luminal surface of the endothelium. This carbohydrate-rich layer mainly consists of proteoglycans (PGs) and glycoproteins $(G P s)^{11-13}$. The PGs and GPs are considered the 'backbone' molecules as they provide the connection between the glycocalyx and endothelium ${ }^{12}$. PGs in the glycocalyx contain a core protein. These core proteins are either firmly connected to the endothelial cell membrane (i.e. syndecan, glypican) or are more soluble and reside in the glycocalyx mesh or diffusely in the blood stream (i.e. mimecan, perlecan, biglycan) ${ }^{12}$.

As 'backbone' molecules, the PGs and GPs provide a network in which plasma- or endothelium-derived soluble molecules are incorporated. These soluble molecules are interconnected directly or indirectly to the core proteins via GPs and glycosaminoglycan (GAG) side chains. Ninety percent of the glycocalyx's GAG side chains are composed of heparan sulfate and hyaluronan ${ }^{12,14-16}$. Because many of the GAG side chains are highly sulfated, the glycocalyx surface that is exposed to the bloodstream is negatively charged $^{12}$. The layer composed of PGs, GPs, GAG side chains and containing soluble molecules, is not a static structure because soluble molecules are replaced, shedded and synthesized continuously. This more dynamic part of the glycocalyx is located near the plasma ${ }^{12}$. 


\subsection{Role of the glycocalyx}

\subsubsection{Barrier function}

The glycocalyx has a number of functions. First of all, it provides a mechanical barrier between plasma and endothelium as it reduces access of plasma components to the endothelial surface ${ }^{17}$. Erythrocytes and thrombocytes can barely penetrate the glycocalyx. Since thrombocytes cannot attach to endothelial procoagulant peptides, thrombocyte aggregation is inhibited. Furthermore, many anticoagulant molecules reside in the glycocalyx and contribute to the thromboresistant nature of the endothelium $^{12,18}$. An intact and stable glycocalyx suppresses interaction between plasma cells and endothelial cells. By example, leucocyte adhesion to cell-adhesion molecules, like the vascular cell-adhesion molecules (VCAM) and intercellular adhesion molecules (ICAM), is reduced by the glycocalyx ${ }^{19,20 .}$ It was also demonstrated that acute coagulopathy following trauma was associated with increased plasma levels of syndecan-1, a glycocalyx core protein ${ }^{21,22}$. This finding suggests shedding of the glycocalyx following trauma, resulting in a decreased glycocalyx thickness and thus increased availability of procoagulant peptides ${ }^{22}$. Finally, the negatively charged glycocalyx surface forms an electrostatic barrier for plasma cells and proteins, like albumin $^{12}$.

\subsubsection{Mechanotransduction of shear stress}

The glycocalyx plays a crucial role in mechanotransduction in response to shear stress. It has been shown that GAG side chains, like heparan sulfate, are important shear sensors $^{23}$. Shear stress is subsequently signalled to the endothelium via core proteins. This leads to remodelling of the endothelial cell, induced by syndecan- $1^{24}$. Another core protein, glypican-1, is involved in the activation of endothelial nitric oxide synthase (eNOS) which mediates nitric oxide (NO) production. Finally, NO induces vasodilatation, reducing shear stress ${ }^{25,26}$. In case of persisting shear stress, vascular inflammation occurs, which subsequently leads to atherosclerosis ${ }^{13,25}$. Therefore, an adequate response to shear stress is important to for maintaining healthy vasculature.

\subsubsection{Reducing oxidative stress}

Another protective property of the glycocalyx is its capacity to diminish activity of oxygen radicals by scavenging enzymes, like extracellular superoxide dismutase ${ }^{12,27}$. This process reduces oxidative stress and maintains NO bioavailability, ultimately preventing endothelial dysfunction.

The glycocalyx is a vulnerable layer that is easily disrupted. Disruption leads to increased permeability for plasma proteins and exposure of endothelial cell adhesion molecules to the plasma, which in turn triggers adhesion and extravasation of leucocytes and platelets resulting in local inflammation, edema, platelet aggregation, 
increased oxidative stress and loss of vascular responsiveness ${ }^{11,19,28-30}$. On the other hand, the glycocalyx can be quickly reconstituted, restoring its protective abilities ${ }^{15,30,31}$.

\subsection{Glycocalyx in disease}

Since it is thought that preservation of glycocalyx thickness is an important aspect of vascular health, a decreased glycocalyx thickness may result in vascular disease ${ }^{32}$. In fact, vascular disease related risk factors like atherosclerosis, hypertension and diabetes mellitus, but also specific diseases like lacunar stroke, renal failure, chronic infectious disease and sepsis are all associated with decreased glycocalyx thickness ${ }^{2,3,33-41}$. Possible mechanisms of glycocalyx disruption include a direct effect of circulating cytokines, local ischemia, and hypoxia/reperfusion ${ }^{28,38,42}$. Thus, a vast amount of studies has shown that disrupted microcirculation in general and a disrupted glycocalyx in particular, may be involved in the pathophysiology of vascular, renal, infectious and endocrine diseases s,3,35,36,39,41. $^{1}$.

As mentioned, the glycocalyx is a significant determinant of vascular permeability, and could be a key component of blood-brain barrier permeability as part of the cerebral microcirculation ${ }^{12,29,33}$. The hypothetical various barrier roles of the glycocalyx as a component of the blood-brain barrier are illustrated in Figure 2.1. In Figure 2.2, the possible consequences of a disrupted glycocalyx for blood-brain barrier permeability is illustrated.

Mechanisms of a disrupted blood-brain barrier play an important pathophysiological role in neurological diseases like multiple sclerosis, stroke, Alzheimer's disease, vascular dementia, cerebral small vessel disease, traumatic brain injury and epilepsy ${ }^{4-10,33,43-45}$. In all of these diseases, inflammation, edema, oxidative stress or endothelial dysfunction are described. Moreover, important risk factors for stroke, like hypertension and diabetes mellitus are associated with decreased glycocalyx function ${ }^{33,46-49}$. Remarkably, a specific role for cerebrovascular glycocalyx dysfunction in these diseases has not been evaluated to date.

In this review, we report on the different techniques to visualize, qualify and quantify the glycocalyx and how some of these techniques may be used to study the cerebrovascular glycocalyx. 


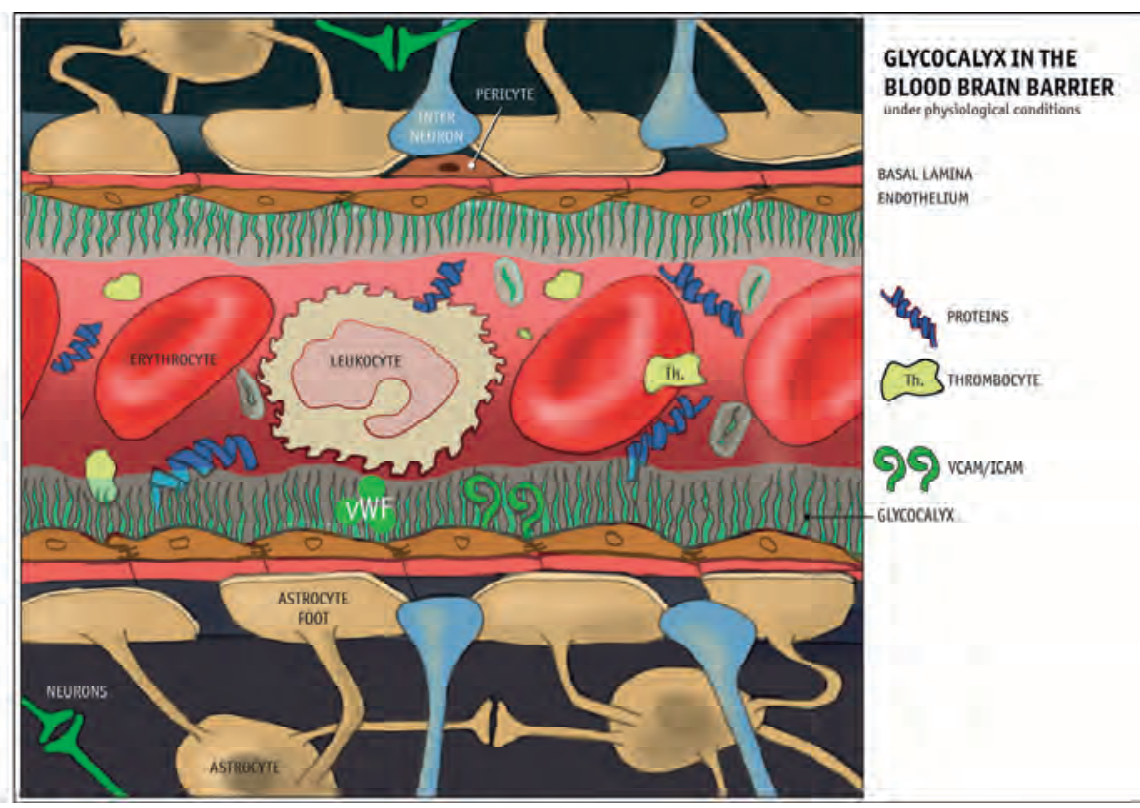

Figure 2.1 Barrier function of the glycocalyx as part of the blood-brain barrier

The possible configuration of the cerebrovascular glycocalyx as component of the blood-brain barrier is illustrated. The glycocalyx aligns the endothelial cells, which are paracellularly interconnected by tight junctions. Important endothelial receptor molecules like ICAM and VCAM reside within the glycocalyx, just like by example the von Willenbrand factor (VWF). The endothelial cells are surrounded by the basal lamina that variably contains pericytes. The astrocyte foot processes ensheath the entire cerebral capillary network and are intertwined with interneurons. Clearly, plasma cellular and macromolecular components cannot attach to the endothelial surface components due to the presence of the glycocalyx. In this way, leukocyte adhesion, thrombocyte aggregation and the extravasation of plasma proteins is prohibited. Also, endothelial cells are not directly exposed to the plasma flow reducing shear stress.

\section{Techniques to study the glycocalyx}

\subsection{Experimental (video-)microscopy techniques}

\subsubsection{Transmission electron microscopy}

The first images of the glycocalyx were made using transmission electron microscopy (TEM) in $1966^{50}$. Currently, the use of TEM for glycocalyx assessment is limited as it can only visualize the glycocalyx in vitro after extensive preparation procedures including fixation, dehydration, sectioning and staining, that affect constituents of the dynamic part of the glycocalyx. Therefore, TEM actually implies visualization of the membranebound components of the glycocalyx ${ }^{51}$. 


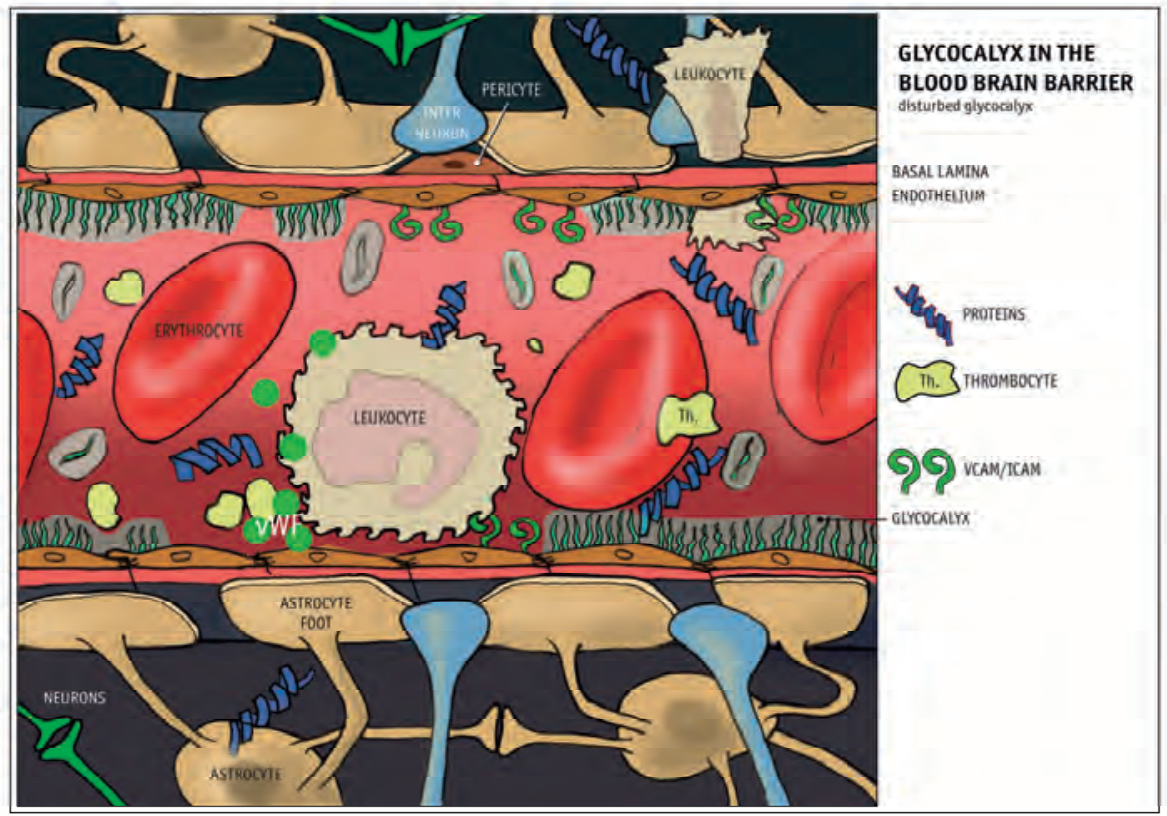

Figure 2.2 Figure 2: The consequences of a disrupted glycocalyx.

Here, the hypothesized effects of a disrupted glycocalyx at the level of the blood-brain barrier are shown. Leukocytes can now attach to the leukocyte adhesion molecules propagating local inflammation. Due to the availability of platelet activating factors, thrombocyte aggregation is stimulated. Moreover, plasma proteins may be transported across the blood-brain barrier. As a consequence of these changes, local inflammation, edema and different vascular responsiveness are induced, directly affecting the blood-brain barrier properties. Noteworthy, the red blood cell column (RBCC) broadens, as it approaches the endothelial cell layer more closely which is of importance for diverse indirect glycocalyx analysing methods.

Thus, the challenge of in vitro TEM is to preserve and stain the total glycocalyx, and even though perfusion-fixation techniques may theoretically enable preservation of the complete glycocalyx, limitations remain. This is related to the fact that during sample processing, dehydration artefacts and extraction of low molecular weight substances causes the glycocalyx to collapse ${ }^{52,53}$. This problem can be partly overcome by rapid freezing and freeze substitution (RF/FS). In order to reduce ice crystal formation, tissue is quickly frozen under high pressure conditions ${ }^{52,54}$. By placing the frozen specimen in organic solvents like acetone containing osmium tetroxide, the ice is replaced by these solvents ${ }^{32,52,55}$. Using RF/FS, Ebong et al. measured a 10-100 times thicker cultured glycocalyx compared to conventional TEM without RF/FS ${ }^{52}$.

Another limitation of TEM is the fact that it cannot distinguish between glycocalyx and adjacent tissue, because of the poor interaction of the glycocalyx's highly polysaccharide content with post-fixation stains ${ }^{51,56}$. In his 1966 paper, Luft was the first to actually image the glycocalyx by using a ruthenium red stain with osmium tetroxide 
resulting in an electron-rich stain showing a $20 \mathrm{~nm}$ thick glycocaly $\mathrm{x}^{50}$. In 2003, van den Berg et al. introduced a new staining protocol using alcian blue $8 \mathrm{GX}$, resulting in glycocalyx thicknesses between 200 and $500 \mathrm{~nm}$, as can be seen in Figure $2.3^{12,57}$. Recently, this staining protocol has been used for the evaluation of the glycocalyx in aqueous pore outflow tracts in the bovine and human eye ${ }^{26}$.

Thus, optimized preservation techniques and the introduction of novel stainings are promising steps for in vitro glycocalyx research using TEM. However, these techniques remain time consuming and do not allow functional experiments.

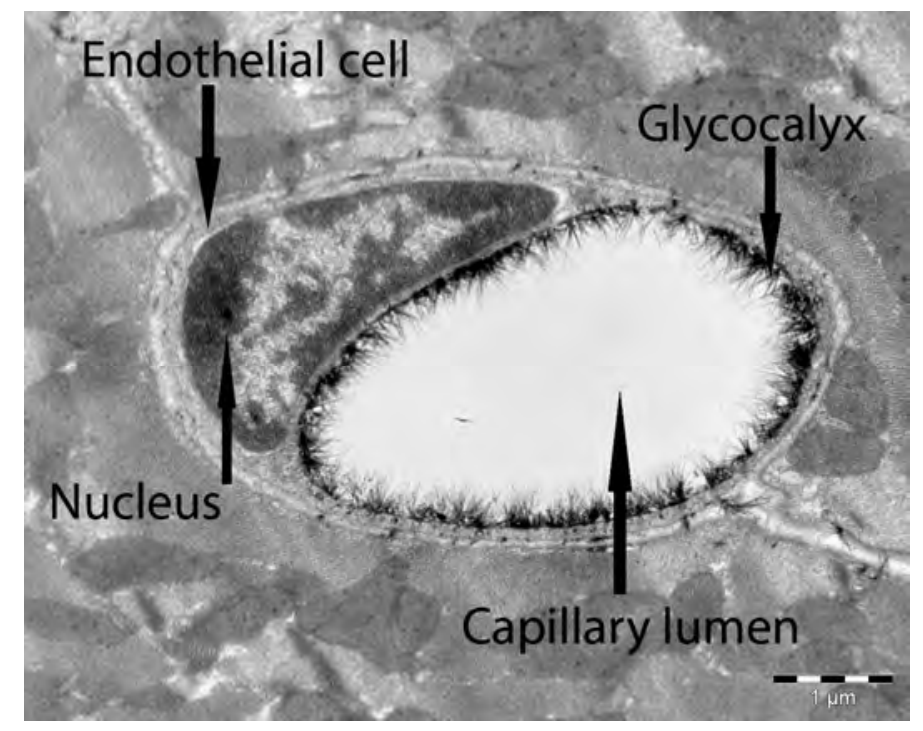

Figure 2.3 Transmission electron microscopic image of the glycocalyx.

TEM image of the glycocalyx in a left ventricular myocardial capillary following Alcian blue 8GX staining. The capillary lumen is enclosed by the endothelial cell. The luminal side of the endothelium is aligned by a polysaccharide layer containing proteoglycans (PG) and glycoproteins (GP). This is the static, membrane-bound part of the glycocalyx. The dynamic part, containing the plasma constituents, is not visualized due to collapse following the preparation and staining procedures.

\subsubsection{Fluorescent microscopy techniques}

\subsubsection{Intravital microscopy}

The most commonly used microscopy technique for glycocalyx assessment is fluorescence intravital microscopy (IVM). This technique allows recognition of the glycocalyx defined as an exclusion zone between erythrocytes and the endothelium, an indirect analysis technique to determine glycocalyx thickness. This exclusion zone is reviewed more thoroughly in paragraph 3. 
Fluorescence IVM has been proven to be a useful in vivo technique in easily accessible vessels, like in cremaster muscle and dorsal skinfold chamber ${ }^{29,42,58-61}$. Clinical application of non-fluorescent IVM is however limited to the nailfold's glycocalyx due to limited tissue penetration and toxicity of fluorescent dyes ${ }^{36,62-64}$. Moreover, it is limited to microvessels less than $15 \mu \mathrm{m}$ in diameter. This is due to the optical difficulties associated with focusing on the boundary of the fluorescent isothiocyanate (FITC)dextran column in larger microvessels and to the limited penetration of the excitation light ${ }^{65,66}$.

Using IVM, glycocalyx thickness of $0.4-0.5 \mu \mathrm{m}$ has been found in skeletal muscle capillaries with a diameter of $5.1 \pm 0,1 \mu \mathrm{m}$. This is comparable to results from another study in which binding of fluorescent lectins, see next paragraph, indicated that the capillary's in vivo glycocalyx may be $0.45 \mu \mathrm{m}$ thick ${ }^{67}$.

\subsubsection{Confocal and two-photon laser scanning microscopy}

Confocal laser scanning microscopy (CLSM) and two-photon laser scanning microscopy (TPLSM) are two microscopy techniques that are less subsidiary to fragility and instability than TEM. Both techniques require fluorescent labeling, for which lectins have mainly been used. Lectins are carbohydrate proteins that bind to specific disaccharide moieties of GAG chains ${ }^{41,69,70}$. The most frequently used lectins for glycocalyx research are lycopersicon esculentum agglutinin (LEA) and wheat germ agglutinin (WGA), which both label glucosamine-based glycosaminoglycans like heparan sulfate and hyaluronan ${ }^{69,71}$. Fluorescent-labelled lectins are useful for quantitative analysis of glycocalyx dimensions like thickness, density and continuity.

Antibodies against specific carbohydrate domains on heparan sulfate and hyaluronan have been developed over recent years, while also antibodies against core-proteins like syndecan, glypican and perlecan are available $\mathrm{s}^{23,41,70,72}$. Using these antibodies, specific components of the glycocalyx could be analyzed qualitatively.

Fluorescent lectins attached to the glycocalyx can be visualized using CLSM. CLSM offers optical sectioning and 3D reconstruction of vessels, but tissue penetration is limited and resolution quickly deteriorates with increasing depth ${ }^{66,73}$. In atherosclerotic carotid arteries of mice, resolution of CLSM was superior to TPLSM at a depth of $15 \mu \mathrm{m}$, but CLSM resolution quickly deteriorated resulting in blurry images at a depth of $40 \mu^{73}$. In comparison, at $80 \mu \mathrm{m}$ below the outer surface, endothelial cells were still visible in the TPLSM image without bleaching ${ }^{73}$. Phototoxicity and photodamage also restrict the use of CLSM. Therefore, CLSM is limited to small isolated vessels and vessels in thin, less than $20 \mu \mathrm{m}$, tissue in vivo.

As was shown, TPLSM offers deeper tissue penetration, higher resolution, and provides better optical sectioning and less phototoxicity. Instead of one blue photon as in CLSM, TPLSM takes advantage of the simultaneous absorption of two near infrared photons resulting in the excited emitting state of fluorescent molecules ${ }^{12,66,74,75}$. The detected fluorescent emission light always originates from the focal position, independent of 
scattering, because out-of-focus absorption and fluorescence are absent ${ }^{66}$. Due to the wavelength of $800 \mathrm{~nm}$, excitation light scattering is significantly less and tissue penetration is improved ${ }^{66,69}$. Therefore, TPLSM for glycocalyx evaluation has mainly been applied to isolated arterioles, venules and arteries. Recently, a multiphoton microscopy device with hand-held probe has been developed which further increases possibilities for in vivo imaging ${ }^{75,76}$.

Megens et al. reported on glycocalyx thickness of $4.5 \pm 1.0 \mu \mathrm{m}$ in rat common carotid arteries (mean diameter $245 \mu \mathrm{m}$ ) using TPLSM ${ }^{66}$.

\subsubsection{Microparticle image velocimetry}

Smith et al. suggested another exclusion zone technique using microparticle image velocimetry $(\mu-P I V){ }^{77}$. Visualization of infused fluorescent microspheres is performed using stroboscopic double flash epi-illumination. This technique enables estimation of microsphere-velocity inside the central lumen of a vessel or in the near wall region before and after dye treatment to degrade the glycocalyx. Velocity vanishes at a radial location that is approximately $500 \mathrm{~nm}$ away from the endothelial cell surface rather than at the endothelial cell surface ${ }^{77-80}$. This is comparable to the thickness of the endothelial glycocalyx as measured by IVM. $\mu$-PIV has only been used in vessels larger than $20 \mu \mathrm{m}^{77-80}$. Application of $\mu$-PIV is limited to preclinical experiments due to toxicity of the microspheres.

\subsection{Clinical (video-)microscopy techniques}

\subsubsection{Orthogonal polarization spectral imaging}

Videomicroscopy techniques based on erythrocyte exclusion zones are nowadays frequently used for glycocalyx observations. In 1987, orthogonal polarization spectral (OPS) imaging was introduced by Slaaf et al., and since 1999, a hand-held version of this technique has been available ${ }^{81,82}$. In OPS imaging, the subject medium is illuminated with plane polarized light of a $548 \mathrm{~nm}$ wavelength. This light is absorbed by oxy- and deoxyhemoglobin in erythrocytes. The remitted light is imaged in an orthogonal plane and passes through a spectral filter for wavelength isolation and is then linearly polarized again towards the objective lens ${ }^{3,83}$. The objective lens shows an image of the illuminated region upon a handheld charge-coupled device (CCD) video camera ${ }^{36,63,81,84}$. In healthy vascular circumstances, the red blood cell column (RBCC) is not able to compress the glycocalyx. This is in contrast with leukocytes, that easily compress the glycocalyx to $20-25 \%$ of its undisturbed thickness ${ }^{15,67,85}$. Glycocalyx thickness can therefore be determined by calculating the difference of the RBCC shortly before and directly after leucocyte passage. Also, using OPS imaging the glycocalyx can be estimated by the perfused boundary region (PBR). The PBR is another indirect glycocalyx measurement gauge based on the variation of the RBCC width. Both these 
indirect glycocalyx analysis techniques are discussed more thoroughly in the third paragraph.

Since glycocalyx analysis in OPS imaging is based on RBCC variations, fluorescent dyes are not required. This enables clinical assessment of the glycocalyx. Using the handheld small optical probe, one can easily visualize the glycocalyx of superficial capillaries of external tissue structures like the sublingual mucosa, skin, nailfold, skeletal muscle, conjunctiva, and gingiva, ${ }^{1,83,86}$. Glycocalyx assessment has also successfully been performed in lungs, liver and intestines in animal studies ${ }^{63,83,84,87,88}$. OPS imaging is painless, well tolerated and takes only a few minutes ${ }^{15,81}$.

Results of glycocalyx measurement using OPS imaging are comparable to IVM $^{15,62,85,86}$. The intersession variation coefficiency has been estimated to be around $15 \%{ }^{15}$. Interand intra-observer reliability using semi-automatic glycocalyx thickness measurement software increases reliability of videomicroscopy ${ }^{89}$.

Like IVM, OPS imaging is prone to motion and pressure artifacts. Motion-induced image blurring by movement of the OPS device, the tissue and flow of erythrocytes can cause suboptimal imaging of the capillaries $3,36,83,90$.

\subsubsection{Sidestream darkfield and oblique imaging}

In sidestream darkfield (SDF) imaging and oblique imaging, incorporated in Capiscope imaging, illumination is provided by concentrically placed light emitting diodes (LEDs) surrounding the central light guide with the magnifying lens. Since the lens is isolated from the illuminating outer ring, contamination of the image by remitted light of tissue surface reflections is prevented. Light from the illuminating outer ring penetrates the tissue and illuminates the tissue-embedded microcirculation by scattering. The LEDs provide a pulsed green light synchronously with the CCD frame rate, preventing motion-induced artifacts ${ }^{64,83,90}$. Moreover, the LEDs emit at a central wavelength of $530 \mathrm{~nm}$, which is absorbed by (de-) oxyhemoglobin in erythrocytes. As a result, erythrocytes appear dark surrounded by a grayish background, as can be seen in Figure 2.4 and video $1^{83,90}$. Consequently, tissue reflections are avoided resulting in clearer images ${ }^{2}$. In SDF and oblique imaging, the glycocalyx is determined by RBCC width variation in a comparable fashion as in OPS imaging.

SDF and oblique imaging is also implemented in a handheld video microscope and comes with the same clinical benefits as OPS imaging. They have been validated by comparing it to OPS imaging, and come with significantly better contrast and resolution, and reduced motion-induced image blurring ${ }^{90}$. Image acquisition and data analysis of SDF and oblique imaging are automatically mediated using specific software ${ }^{91}$.

\subsection{Plasma markers}

Constituents of the glycocalyx are continuously replaced, shedded and synthesized locally $^{12}$. Disruption of the glycocalyx in response to hyperglycemia, reactive oxygen 
species, tumor-necrosis factor alpha or other inflammatory mediators increases the release of these components ${ }^{16,31,34,42,51,92}$. Therefore, plasma levels of shedded glycocalyx core proteins and main GAG chains could be markers of glycocalyx stability and thickness.

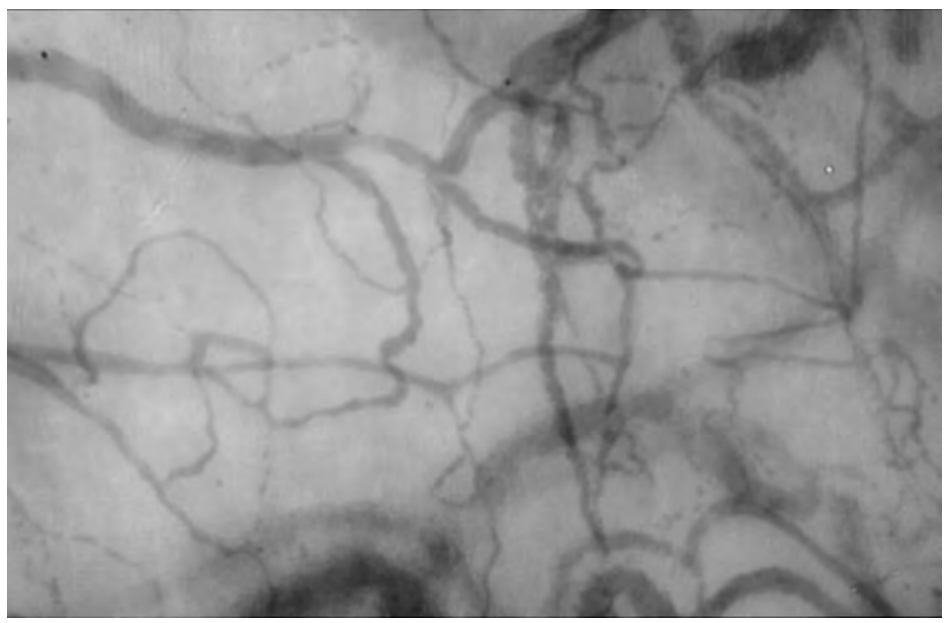

Figure $2.4 \quad$ Image of sublingual microvasculature.

In this figure, the sublingual microvasculature in a healthy person is demonstrated by using SDF/oblique imaging. Vessels can be seen on a grayisch background. Within the vessels, erythrocytes are visualized and the red blood cell column (RBCC) can be calculated.

Hyaluronan serves as a mechano-shear sensor regulating nitric oxide release and maintaining vascular permeability ${ }^{34,93}$. The high molecular weight (HMW) hyaluronan chains possess an anti-inflammatory, antiangiogenic and immunosuppressive effect and prevent leucocyte adhesion related to their gel-like properties ${ }^{13,51}$. Inflammation induced release of hyaluronidase results in cleavage of HMW hyaluronan chains and formation of low molecular weight (LMW) hyaluronan chains that do not have adhesion prevention properties ${ }^{94}$. Increased plasma levels of hyaluronan and hyaluronidase have been found in dialysis patients in which also an increased PBR was demonstrated using SDF imaging ${ }^{34}$.

Heparan sulfate has also been used as glycocalyx marker. Heparan sulfate is an important mechano-shear sensor, and is involved in inflammatory processes, as tumornecrosis factor alpha increases heparan sulfate domains involved in leucocyte adhesion and extravasation ${ }^{20,95-97}$. A multifold increase of heparan sulfate plasma concentrations has been found in patients following perioperative global or regional ischemia ${ }^{98}$. Using TEM, a decreased glycocalyx thickness was found in these patients. However, results showed a high inter-individual variability probably originating from varying individual glycocalyx thickness and different turnover values. 
In the same study, plasma levels of syndecan-1 were evaluated. Shedding of syndecan-1 has been observed following proteolytic and oxidative mechanisms ${ }^{34}$. Multiple studies have found multifold increased levels of syndecan-1 in disease state or during surgery ${ }^{34,40,98}$. Additionally, increased levels of syndecan-1 are associated with inflammation and coagulopathy, pathological findings found when the glycocalyx is damaged $^{40}$. Again, results showed a high inter-individual variability making interpretation of these results challenging.

Thus, glycocalyx marker detection is a safe and simple method for analysis of glycocalyx constituent plasma levels. However, results lack sensitivity and specificity, and show a high inter-individual variability.

\section{Analyzing glycocalyx thickness}

Glycocalyx analysis in TEM, CLSM and TPLSM is based on staining of the glycocalyx. In this way quantitative, i.e. glycocalyx thickness, and qualitative, i.e. staining of specific glycocalyx components, analysis can easily be performed. This encompasses mainly in vitro analysis.

In preclinical glycocalyx experiments one can take advantage of the 'exclusion zone' originating from application of fluorescent macromolecules or microspheres. Since the glycocalyx is impermeable to albumin, fluorescent macromolecules like 70 kDa dextran, which has a molecular weight comparable to that of albumin can be applied to determine glycocalyx thickness as a fluorescence exclusion zone ${ }^{58,68}$. This exclusion zone is also found when fluorescent microspheres are visualized using stroboscopic double flash epi-illumination. This technique is based on estimation of microspherevelocity inside the central lumen of a vessel or in the near wall region before and after dye treatment to degrade the glycocalyx. Velocity has been found to vanish at a radial location approximately $500 \mathrm{~nm}$ away from the endothelial cell surface rather than at the endothelial cell surface ${ }^{77-80}$. In this way, the glycocalyx can be assessed in preclinical conditions.

Another way to define the clinical glycocalyx as an 'exclusion zone' is by red blood cell column (RBCC) variation slightly before and directly after leukocyte passage. The RBCC is not able to significantly compress the glycocalyx. This is in contrast with leukocytes, that easily compress the glycocalyx to $20-25 \%$ of its undisturbed thickness ${ }^{15,67,85}$. Only core proteins are able to withstand the compressing pressure due to a high flexural rigidity that is large enough to restore the compressed part after leucocyte passage ${ }^{85}$. This recoil of the glycocalyx after leucocyte compression takes a short time during which the RBCC is widened ${ }^{85}$. Leukocyte passage does not result in RBCC widening when glycocalyx thickness is significantly degraded ${ }^{15,85}$. Thus, RBCC width difference between post-leucocyte passage and pre-leucocyte passage is a measure of the compressibility of the glycocalyx. Next, in the two-dimensional plane the glycocalyx thickness can be calculated using the RBCC shortly before and directly after leucocyte 
passage as: (post-leucocyte RBCC - pre-leucocyte RBCC) $/ 2^{15,89}$. Due to the core protein flexural rigidity, this is an underestimation of glycocalyx thickness that is approximately $30 \%{ }^{15,85}$

In recent years, the perfused boundary region (PBR) has been the primary glycocalyx gauge in many glycocalyx studies, mainly involving the sublingual superficial microcirculation ${ }^{33,90,99,100}$. The PBR is also based on RBCC width variation. An unstable or damaged glycocalyx is more accessible for erythrocytes resulting in increased RBCC width $^{15,101}$. Thus, an increase in RBCC width is a gauge for glycocalyx damage. Subsequently, the total perfused diameter (Dperf) is determined by a linear regression analysis of the measurements between the $25^{\text {th }}$ and $75^{\text {th }}$ percentile of the RBCC ${ }^{91}$. The median RBCC width is calculated by the $50^{\text {th }}$ percentile of RBCC column width. From this the PBR follows from the calculation: (Dperf - mean RBCC width) $/ 2^{91,102}$.

Clinical glycocalyx analysis using IVM, OPS imaging or SDF/oblique imaging is performed using the exclusion zone based on RBCC or PBR. However, these analysis techniques remain an indirect measurement of the glycocalyx.

\section{Cerebrovascular glycocalyx}

In vitro characterization of the cerebrovascular glycocalyx has been performed using TEM and CLSM $^{103-105}$. In these studies, different lectins were visualized to determine staining patterns. Using TEM, Mazetti et al. demonstrated the glycocalyx in guinea pigs by the significant binding of WGA and LEA at the luminal side of the endothelium. This luminal lining was continuous with the endothelium but thickness was not provided ${ }^{104}$. Hempel et al. provided images of mice cerebrovascular glycocalyx and quantified the glycocalyx area and continuity along the vessel wall ${ }^{49}$. A significant decline in area per vessel length was found in cerebral malaria infected mice compared to controls. Moreover, they quantified serum hyaluronan and found higher levels in the malaria infected mice than in controls.

To the best of our knowledge, clinical visualization of the cerebrovascular glycocalyx has not been performed to date. However, other microcirculatory parameters like erythrocyte flow velocity and functional capillary density have been assessed using fluorescent IVM. Clear images of erythrocytes, the vascular wall and fluorescent dye permeability were obtained in rat and pig brains under various conditions ${ }^{106-111}$. By example, Hudetz et al. have found an erythrocyte flow velocity in capillaries of $0.6-0.7 \mathrm{~mm} / \mathrm{s}$, which significantly increased when hypoxia or hypercapnia was induced $^{109}$.

The many studies on cerebral microcirculation dynamics using several of the above described techniques show promising results, seemingly demonstrating the feasibility of in vivo cerebral glycocalyx visualization. Most of the cerebral microcirculation observation studies have been using OPS imaging. Observed microcirculatory parameters included capillary perfusion, microcirculation flow velocity, vessel diameter 
and capillary functional density, in experiments on the cerebral microvasculature response to cardiopulmonary arrest or following brain injury ${ }^{63,87,112-115}$. Moreover, the human cerebral microcirculation has been visualized during intracranial surgery for arteriovenous malformations, aneurysms, and tumour resection using OPS imaging $^{84,116,117}$.

As SDF imaging has been validated against OPS imaging for microcirculatory parameters, it has been used for cerebral microcirculation evaluation shortly after its introduction $^{90}$. Vessel density, vessel diameter, and vessel perfused density have been assessed in preclinical conditions ${ }^{118-120}$. Pérez-Bárcena et al. have successfully performed SDF imaging on cortical vessels during decompressive brain surgery for stroke and traumatic brain injury, and compared these data to control patients undergoing brain surgery for subcortical brain tumours ${ }^{121,122}$. Vessels with diameters between 10-100 $\mu \mathrm{m}$ were visualized with high resolution. In the control group, vessel density and perfused vessel density in vessels smaller than $25 \mu \mathrm{m}$ were found to be $99.5-100 \%$ and $6.16-6.5 \mathrm{~mm}$ vessel $/ \mathrm{mm}^{2}$, respectively ${ }^{121,122}$. Thus, cerebral microcirculation parameters can be assessed by SDF imaging.

As mentioned, visualization of the in vivo cerebrovascular glycocalyx has not been performed to date. The abovementioned results on the observation of microcirculatory parameters support our believe that the current state-of-the-art microscopy is able to evaluate the human cerebrovascular glycocalyx in vivo. For clinical application of these existing techniques, only additional analysis software has to be applied to current OPS or SDF imaging.

\section{Conclusion}

The glycocalyx is of crucial importance for endothelial function. A damaged glycocalyx has been found in many vascular diseases including stroke $e^{1,2,22,33,34,36,40,46}$. Moreover, the glycocalyx is a significant determinant of vascular permeability and may also determine blood-brain barrier permeability. Increased blood-barrier permeability has been associated with many neurological conditions like stroke and epilepsy ${ }^{4-8,43-45}$. Remarkably, only two studies have been published that focused on the cerebrovascular glycocalyx ${ }^{33,49}$.

In this review, we have described the available techniques to evaluate the cerebrovascular glycocalyx. We believe different study conditions, i.e. preclinical versus clinical and in vitro versus in vivo, require different imaging qualities. Based on the pros and cons, summarized in Table 2.1, we think that TPLSM is the preferred imaging technique for in vitro glycocalyx imaging. For in vivo preclinical visualization of the cerebrovascular glycocalyx IVM, TPLSM or SDF/oblique imaging are recommended. We think that the human cerebrovascular glycocalyx is best visualized in vivo by SDF imaging, or a similar technique like oblique imaging. It could easily be applied intracranially, although this has not been reported on in current literature. 
40 $\mid$ Chapter 2

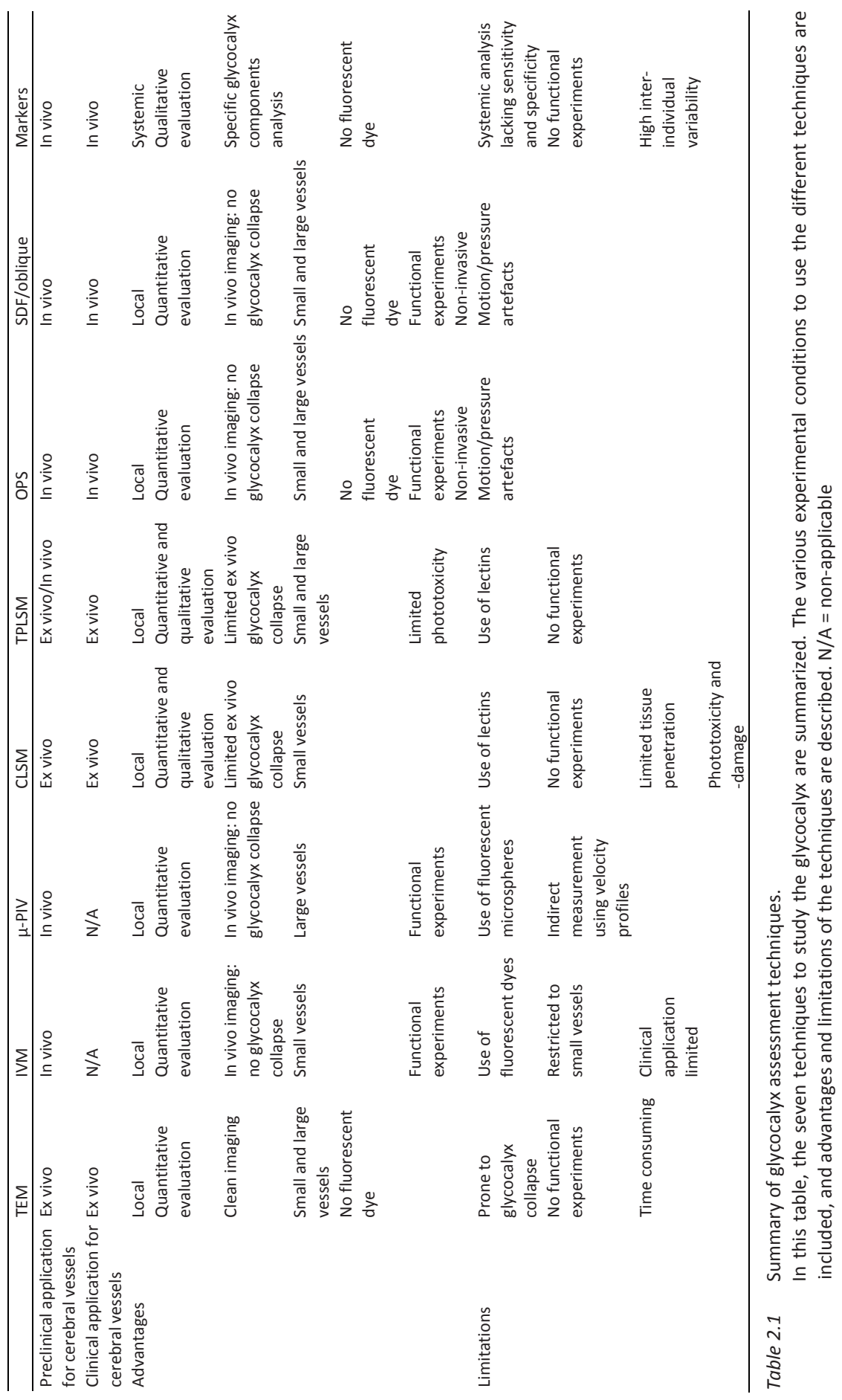




\section{References}

1. Zuurbier CJ, Demirci C, Koeman A, Vink H, Ince C. Short-term hyperglycemia increases endothelial glycocalyx permeability and acutely decreases lineal density of capillaries with flowing red blood cells. J Appl Physiol. 2005;99:1471-6.

2. Ince C. The microcirculation is the motor of sepsis. Crit Care. 2005;9 Suppl 4:S13-9.

3. Lindert J, Werner J, Redlin M, Kuppe H, Habazettl H, Pries AR. OPS imaging of human microcirculation: A short technical report. J Vasc Res. 2002;39:368-72.

4. van Vliet EA, Aronica E, Gorter JA. Role of blood-brain barrier in temporal lobe epilepsy and pharmacoresistance. Neuroscience. 2014;277:455-73.

5. Bell RD, Zlokovic B V. Neurovascular mechanisms and blood-brain barrier disorder in Alzheimer's disease. Acta Neuropathol. 2009;118:103-13.

6. Palmer AM. The role of the blood brain barrier in neurodegenerative disorders and their treatment. J Alzheimers Dis. 2011;24:643-56.

7. de Vries HE, Kooij G, Frenkel D, Georgopoulos S, Monsonego A, Janigro D. Inflammatory events at blood-brain barrier in neuroinflammatory and neurodegenerative disorders: implications for clinical disease. Epilepsia. 2012;53 Suppl 6:45-52.

8. Ortiz GG, Pacheco-Moisés FP, Macías-Islas MÁ, et al. Role of the blood-brain barrier in multiple sclerosis. Arch Med Res. 2014;45:687-97.

9. Ueno $\mathrm{M}$, Chiba $\mathrm{Y}$, Matsumoto $\mathrm{K}$, et al. Blood-brain barrier damage in vascular dementia. Neuropathology. 2016;36(2):115-24

10. Farrall AJ, Wardlaw JM. Blood-brain barrier: ageing and microvascular disease--systematic review and meta-analysis. Neurobiol Aging. 2009;30:337-52.

11. Alphonsus CS, Rodseth RN. The endothelial glycocalyx: A review of the vascular barrier. Anaesthesia. 2014;69:777-84.

12. Reitsma S, Slaaf DW, Vink H, Van Zandvoort MAMJ, Oude Egbrink MGA. The endothelial glycocalyx: Composition, functions, and visualization. Pflugers Arch Eur J Physiol. 2007;454:345-59.

13. Weinbaum S, Tarbell JM, Damiano ER. The structure and function of the endothelial glycocalyx layer. Annu Rev Biomed Eng. 2007;9:121-67.

14. Pries AR, Secomb TW, Gaehtgens P. The endothelial surface layer. Pflugers Arch. 2000;440:653-66.

15. Nieuwdorp M, Meuwese MC, Mooij HL, et al. Measuring endothelial glycocalyx dimensions in humans: a potential novel tool to monitor vascular vulnerability. J Appl Physiol. 2008;104:845-52.

16. Gao L, Lipowsky HH. Measurement of solute transport in the endothelial glycocalyx using indicator dilution techniques. Ann Biomed Eng. 2009;37:1781-95.

17. Curry FE, Adamson RH. Endothelial glycocalyx: Permeability barrier and mechanosensor. Ann Biomed Eng. 2012;40:828-39.

18. Egbrink MGO, Van Gestel MA, Broeders MAW, et al. Regulation of microvascular thromboembolism in vivo. Microcirculation. 2005;12:287-300.

19. Constantinescu AA, Vink $H$, Spaan JAE. Endothelial cell glycocalyx modulates immobilization of leukocytes at the endothelial surface. Arterioscler Thromb Vasc Biol. 2003;23:1541-7.

20. Rops AL, van den Hoven MJ, Baselmans MM, et al. Heparan sulfate domains on cultured activated glomerular endothelial cells mediate leukocyte trafficking. Kidney Int. 2008;73:52-62.

21. Johansson PI, Sørensen AM, Perner A, et al. Disseminated intravascular coagulation or acute coagulopathy of trauma shock early after trauma? An observational study. Crit Care. 2011;15:R272.

22. Chignalia AZ, Yetimakman F, Christiaans SC, et al. The glycocalyx and Trauma. Shock. 2016;45(4):338-48

23. Florian JA, Kosky JR, Ainslie K, Pang Z, Dull RO, Tarbell JM. Heparan sulfate proteoglycan is a mechanosensor on endothelial cells. Circ Res. 2003;93:e136-42.

24. Ebong EE, Lopez-Quintero S V, Rizzo V, Spray DC, Tarbell JM. Shear-induced endothelial NOS activation and remodeling via heparan sulfate, glypican-1, and syndecan-1. Integr Biol. (Camb). 2014;6:338-47.

25. Tarbell JM, Simon SI, Curry F-RE. Mechanosensing at the Vascular Interface. Annu Rev Biomed Eng. 2014;16:505-32.

26. Yang CYC, Huynh T, Johnson M, Gong H. Endothelial glycocalyx layer in the aqueous outflow pathway of bovine and human eyes. Exp Eye Res. 2014;128:27-33. 
27. Li Q, Bolli R, Qiu Y, Tang XL, Murphree SS, French B a. Gene therapy with extracellular superoxide dismutase attenuates myocardial stunning in conscious rabbits. Circulation. 1998;98:1438-48.

28. Becker BF, Chappell D, Bruegger D, Annecke T, Jacob M. Therapeutic strategies targeting the endothelial glycocalyx: Acute deficits, but great potential. Cardiovasc Res. 2010;87:300-10.

29. Henry CB, Duling BR. Permeation of the luminal capillary glycocalyx is determined by hyaluronan. Am J Physiol. 1999;277:H508-14.

30. Henry CB, Duling BR. TNF-alpha increases entry of macromolecules into luminal endothelial cell glycocalyx. Am J Physiol Heart Circ Physiol. 2000;279:H2815-23.

31. Chappell D, Hofmann-Kiefer K, Jacob M, et al. TNF-alpha induced shedding of the endothelial glycocalyx is prevented by hydrocortisone and antithrombin. Basic Res Cardiol. 2009;104:78-89.

32. de Mesy Bentley KL. An 11- $\mu$ m-thick glycocalyx?: it's all in the technique! Arterioscler Thromb Vasc Biol. 2011;31:1712-3.

33. Martens RJH, Vink H, Van Oostenbrugge RJ, Staals J. Sublingual microvascular glycocalyx dimensions in lacunar stroke patients. Cerebrovasc Dis. 2013;35:451-4.

34. Vlahu CA, Lemkes BA, Struijk DG, Koopman MG, Krediet RT, Vink H. Damage of the Endothelial Glycocalyx in Dialysis Patients. J Am Soc Nephrol. 2012;23:1900-8.

35. Cai B, Fan J, Zeng M, Zhang L, Fu BM. Adhesion of malignant mammary tumor cells MDA-MB-231 to microvessel wall increases microvascular permeability via degradation of endothelial surface glycocalyx. J Appl Physiol. 2012;113:1141-53.

36. Černý V, Turek Z, Pařízková R. Orthogonal polarization spectral imaging. Physiol. Res. 2007;56:141-7.

37. Becker BF, Chappell D, Jacob M. Endothelial glycocalyx and coronary vascular permeability: The fringe benefit. Basic Res Cardiol. 2010;105:687-701.

38. Redon J, Rodicio JL. Microalbuminuria in type 1 diabetes mellitus. J Hypertens. 2004;22:1425-8.

39. Padberg JS, Wiesinger A, di Marco GS, et al. Damage of the endothelial glycocalyx in chronic kidney disease. Atherosclerosis. 2014;234:335-43.

40. Ostrowski SR, Berg RMG, Windeløv NA, et al. Coagulopathy, catecholamines, and biomarkers of endothelial damage in experimental human endotoxemia and in patients with severe sepsis: A prospective study. J Crit Care. 2013;28:586-96.

41. Singh A, Fridén V, Dasgupta I, et al. High glucose causes dysfunction of the human glomerular endothelial glycocalyx. Am J Physiol Renal Physiol. 2011;300:F40-8.

42. Kataoka H, Ushiyama A, Kawakami H, Akimoto $\mathrm{Y}$, Matsubara S, lijima T. Fluorescent imaging of endothelial glycocalyx layer with wheat germ agglutinin using intravital microscopy. Microsc Res Tech. 2016;79:31-7.

43. Friedman A. Blood-brain barrier dysfunction, status epilepticus, seizures, and epilepsy: a puzzle of a chicken and egg? Epilepsia. 2011;52 Suppl 8:19-20.

44. Yang Y, Rosenberg GA. Blood-brain barrier breakdown in acute and chronic cerebrovascular disease. Stroke. 2011;42:3323-8.

45. Shlosberg D, Benifla M, Kaufer D, Friedman A. Blood-brain barrier breakdown as a therapeutic target in traumatic brain injury. Nat Rev Neurol. 2010;6:393-403.

46. Sacco RL. Risk factors, outcomes, and stroke subtypes for ischemic stroke. Neurology. 1997;49:S39-44.

47. Kisialiou A, Grella R, Carrizzo A, et al. Risk factors and acute ischemic stroke subtypes. J Neurol Sci. 2014;339:41-6.

48. Vogel J, Sperandio M, Pries AR, Linderkamp O, Gaehtgens P, Kuschinsky W. Influence of the endothelial glycocalyx on cerebral blood flow in mice. J Cereb Blood Flow Metab. 2000;20:1571-8.

49. Hempel C, Hyttel P, Kurtzhals J Al. Endothelial glycocalyx on brain endothelial cells is lost in experimental cerebral malaria. J Cereb Blood Flow Metab. 2014;34:1107-10.

50. Luft JH. Fine structures of capillary and endocapillary layer as revealed by ruthenium red. Fed Proc. 1966;25:1773-83.

51. Dane MJC, van den Berg BM, Lee DH, et al. A microscopic view on the renal endothelial glycocalyx. Am J Physiol Renal Physiol. 2015;308(9):F956-66.

52. Ebong EE, MacAluso FP, Spray DC, Tarbell JM. Imaging the endothelial glycocalyx in vitro by rapid freezing/freeze substitution transmission electron microscopy. Arterioscler Thromb Vasc Biol. 2011;31: 1908-15. 
53. Liu $\mathrm{Y}$, Hidaka $\mathrm{E}$, Kaneko $\mathrm{Y}$, Akamatsu $\mathrm{T}$, Ota $\mathrm{H}$. Ultrastructure of Helicobacter pylori in human gastric mucosa and $\mathrm{H}$. pylori-infected human gastric mucosa using transmission electron microscopy and the high-pressure freezing-freeze substitution technique. J Gastroenterol. 2006;41:569-74.

54. Morphew MK, Morphew MK. Practical methods in high-pressure freezing, freeze-substitution, embedding and immunocytochemistry for electron microscopy. Structure. :1-26.

55. Graham LL, Harris R, Villiger W, Beveridge TJ. Freeze-substitution of gram-negative eubacteria: General cell morphology and envelope profiles. J Bacteriol. 1991;173:1623-33.

56. Erlandsen SL, Kristich CJ, Dunny GM, Wells CL. High-resolution visualization of the microbial glycocalyx with low-voltage scanning electron microscopy: dependence on cationic dyes. J Histochem Cytochem. 2004;52:1427-35.

57. Van den Berg BM, Vink H, Spaan JAE. The endothelial glycocalyx protects against myocardial edema. Circ Res. 2003;92:592-4.

58. Vink H, Duling BR, Capillary endothelial surface layer selectively reduces plasma solute distribution volume. Am J Physiol Heart Circ Physiol. 2000;278:285-9.

59. Harris AG, Hecht R, Peer F, Nolte KM. An improved intravital microscopy system. Int J Microcirc. 1997;17:322-7.

60. Tabuchi A, Mertens M, Kuppe H, Pries AR, Kuebler WM. Intravital microscopy of the murine pulmonary microcirculation. J Appl Physiol. 2008;104:338-46.

61. Torres Filho I, Torres LN, Sondeen JL, Polykratis IA, Dubick M a. In vivo evaluation of venular glycocalyx during hemorrhagic shock in rats using intravital microscopy. Microvasc Res. 2013;85:128-33.

62. Harris AG, Sinitsina I, Messmer K. The Cytoscan Model E-II, a new reflectance microscope for intravital microscopy: Comparison with the standard fluorescence method. J Vasc Res. 2000;37:469-76.

63. Thomale UW, Schaser KD, Unterberg AW, Stover JF. Visualization of rat pial microcirculation using the novel orthogonal polarized spectral (OPS) imaging after brain injury. J Neurosci Methods. 2001;108:8590.

64. Treu CM, Lupi O, Bottino DA, Bouskela E. Sidestream dark field imaging: The evolution of real-time visualization of cutaneous microcirculation and its potential application in dermatology. Arch Dermatol Res. 2011;303:69-78.

65. Potter DR, Damiano ER. The hydrodynamically relevant endothelial cell glycocalyx observed in vivo is absent in vitro. Circ Res. 2008;102:770-6.

66. Megens RTA, Reitsma S, Schiffers PHM, et al. Two-photon microscopy of vital murine elastic and muscular arteries: Combined structural and functional imaging with subcellular resolution. J Vasc Res. 2007;44:87-98.

67. Vink H, Duling BR. Identification of distinct luminal domains for macromolecules, erythrocytes, and leukocytes within mammalian capillaries. Circ Res. 1996;79:581-9.

68. Vink H. Identification of distinct luminal domains for macromolecules, erythrocytes, and leukocytes within mammalian capillaries. Circ Res. 1996;581-9.

69. Reitsma S, Oude Egbrink MGA, Vink H, et al. Endothelial glycocalyx structure in the intact carotid artery: A two-photon laser scanning microscopy study. J Vasc Res. 2011;48:297-306.

70. Singh A, Satchell SC, Neal CR, McKenzie EA, Tooke JE, Mathieson PW. Glomerular endothelial glycocalyx constitutes a barrier to protein permeability. J Am Soc Nephrol. 2007;18:2885-93.

71. Mazzetti S, Frigerio S, Gelati M, Salmaggi A, Vitellaro-Zuccarello L. Lycopersicon esculentum lectin: an effective and versatile endothelial marker of normal and tumoral blood vessels in the central nervous system. Eur J Histochem. 2004;48:423-8.

72. Mulivor AW, Lipowsky HH. Inflammation- and ischemia-induced shedding of venular glycocalyx. Am J Physiol Heart Circ Physiol. 2004;286:H1672-80.

73. Van Zandvoort M, Engels W, Douma K, et al. Two-Photon Microscopy for Imaging of the (Atherosclerotic) Vascular Wall: A Proof of Concept Study. J Vasc Res. 2004;41:54-63.

74. Denk W, Strickler JH, Webb WW. Two-photon laser scanning fluorescence microscopy. Science. 1990;248:73-6.

75. Squirrell JM, Wokosin DL, White JG, Bavister BD. Long-term two-photon fluorescence imaging of mammalian embryos without compromising viability. Nat Biotechnol. 1999;17:763-7.

76. Liu G, Kieu K, Wise FW, Chen Z. Multiphoton microscopy system with a compact fiber-based femtosecond-pulse laser and handheld probe. J Biophotonics. 2011:34-9. 
77. Smith ML, Long DS, Damiano ER, Ley K. Near-wall micro-PIV reveals a hydrodynamically relevant endothelial surface layer in venules in vivo. Biophys J. 2003;85:637-45.

78. Potter DR, Jiang J, Damiano ER. The recovery time course of the endothelial cell glycocalyx in vivo and its implications in vitro. Circ Res. 2009;104:1318-25.

79. Long DS, Smith ML, Pries AR, Ley K, Damiano ER. Microviscometry reveals reduced blood viscosity and altered shear rate and shear stress profiles in microvessels after hemodilution. Proc Natl Acad Sci USA. 2004;101:10060-5.

80. Damiano ER, Long DS, Smith ML. Estimation of viscosity profiles using velocimetry data from parallel flows of linearly viscous fluids: application to microvascular haemodynamics. J Fluid Mech. 2004; 512:119.

81. Groner W, Winkelman JW, Harris a G, et al. Orthogonal polarization spectral imaging: a new method for study of the microcirculation. Nat Med. 1999;5:1209-12.

82. Slaaf DW, Tangelder GJ, Reneman RS, Jäger K, Bollinger A. A versatile incident illuminator for intravital microscopy. Int J Microcirc Clin Exp. 1987;6:391-7.

83. Eriksson S, Nilsson J, Sturesson C. Non-invasive imaging of microcirculation: a technology review. Med Devices (Auckl). 2014;445-52.

84. Pennings FA, Ince C, Bouma GJ. Continuous real-time visualization of the human cerebral microcirculation during AVM surgery using orhogonal polarization spectral imaging. Neurosurgery. 2006;58:167-71.

85. Han Y, Weinbaum S, Spaan JAE, Vink H. Large-deformation analysis of the elastic recoil of fibre layers in a Brinkman medium with application to the endothelial glycocalyx. J Fluid Mech. 2006:217.

86. Mathura KR, Vollebregt KC, Boer K, De Graaff JC, Ubbink DT, Ince C. Comparison of OPS imaging and conventional capillary microscopy to study the human microcirculation. J Appl Physiol. 2001;91:74-8.

87. Wan Z, Sun S, Ristagno G, Weil MH, Tang W. The cerebral microcirculation is protected during experimental hemorrhagic shock. Crit Care Med. 2010;38:928-32.

88. Mutalifu Y, Holm L, Ince C, Theodorsson E, Sjöberg F. Multiple different laminar velocity profiles in separate veins in the microvascular network of brain cortex in rats. Int J Clin Exp Med. 2011;4:10-6.

89. Liuhanen S, Sallisalmi M, Pettilä V, Oksala N, Tenhunen J. Indirect measurement of the vascular endothelial glycocalyx layer thickness in human submucosal capillaries with a plug-in for ImageJ. Comput. Methods Programs Biomed. 2013;110:38-47.

90. Goedhart PT, Khalilzada M, Bezemer R, Merza J, Ince C. Sidestream Dark Field (SDF) imaging: a novel stroboscopic LED ring-based imaging modality for clinical assessment of the microcirculation. Opt Express. 2007;15:15101-14.

91. Lee DH, Dane MJC, Van Den Berg BM, et al. Deeper penetration of erythrocytes into the endothelial glycocalyx is associated with impaired microvascular perfusion. PLoS One. 2014;9(5):e96477

92. Chappell D, Jacob M, Hofmann-Kiefer K, et al. Hydrocortisone preserves the vascular barrier by protecting the endothelial glycocalyx. Anesthesiology. 2007;107:776-84.

93. Mochizuki S, Vink H, Hiramatsu O, et al. Role of hyaluronic acid glycosaminoglycans in shear-induced endothelium-derived nitric oxide release. Am J Physiol Heart Circ Physiol. 2003;285:H722-6.

94. Jiang D, Liang J, Noble PW. Hyaluronan in tissue injury and repair. Annu Rev Cell Dev Biol. 2007;23: 435-61.

95. Rops A, Loeven MA, van Gemst JJ, et al. Modulation of heparan sulfate in the glomerular endothelial glycocalyx decreases leukocyte influx during experimental glomerulonephritis. Kidney Int. 2014;1-11.

96. Norgard-Sumnicht K, Varki A. Endothelial heparan sulfate proteoglycans that bind to L-selectin have glucosamine residues with unsubstituted amino groups. J Biol Chem. 1995;270:12012-24.

97. Rosen SD. Ligands for L-selectin: homing, inflammation, and beyond. Annu Rev Immunol. 2004;22:12956.

98. Rehm M, Bruegger D, Christ F, et al. Shedding of the endothelial glycocalyx in patients undergoing major vascular surgery with global and regional ischemia. Circulation. 2007;116:1896-906.

99. Turek Z, Černý V, Pařízková R. Noninvasive in vivo assessment of the skeletal muscle and small intestine serous surface microcirculation in rat: Sidestream dark-field (SDF) imaging. Physiol Res. 2008;57: 365-71.

100. Petersen SM, Greisen G, Hyttel-Sorensen S, Hahn GH. Sidestream dark field images of the microcirculation: intra-observer reliability and correlation between two semi-quantitative methods for determining flow. BMC Med Imaging. 2014;14:14. 
101. Nieuwdorp M, Mooij HL, Kroon J, et al. Endothelial glycocalyx damage coincides with microalbuminuria in type 1 diabetes. Diabetes. 2006;55:1127-32.

102. Broekhuizen LN, Lemkes BA, Mooij HL, et al. Effect of sulodexide on endothelial glycocalyx and vascular permeability in patients with type 2 diabetes mellitus. Diabetologia. 2010;53:2646-55.

103. Mann DM, Purkiss MS, Bonshek RE, Jones D, Brown AM, Stoddart RW. Lectin histochemistry of cerebral microvessels in ageing, Alzheimer's disease and Down's syndrome. Neurobiol Aging. 1992;13:137-43.

104. Mazzetti S, Librizzi L, Frigerio S, de Curtis M, Vitellaro-Zuccarello L. Molecular anatomy of the cerebral microvessels in the isolated guinea-pig brain. Brain Res. 2004;999:81-90.

105. Neuhaus W, Germann B, Plattner VE, Gabor F, Wirth M, Noe CR. Alteration of the glycocalyx of two blood-brain barrier mimicking cell lines is inducible by glioma conditioned media. Brain Res. 2009;1279:82-9.

106. Duebener LF, Sakamoto T, Hatsuoka S, et al. Effects of hematocrit on cerebral microcirculation and tissue oxygenation during deep hypothermic bypass. Circulation. 2001;104:1260-4.

107. Hudetz AG, Greene AS, Fehér G, Knuese DE, Cowley AW. Imaging system for three-dimensional mapping of cerebrocortical capillary networks in vivo. Microvasc Res. 1993:293-309.

108. Hudetz AG, Fehér G, Weigle CG, Knuese DE, Kampine JP. Video microscopy of cerebrocortical capillary flow: response to hypotension and intracranial hypertension. Am J Physiol. 1995;268:H2202-10.

109. Hudetz AG, Biswal BB, Fehér G, Kampine JP. Effects of hypoxia and hypercapnia on capillary flow velocity in the rat cerebral cortex. Microvasc Res. 1997;54:35-42.

110. Mime L Ben, Arnhold S, Fischer JH, et al. Pharmacologic cerebral capillary blood flow improvement after deep hypothermic circulatory arrest: An intravital fluorescence microscopy study in pigs. J Thorac Cardiovasc Surg. 2005;130:670-6.

111. Cabrales P, Carvalho LJM. Intravital microscopy of the mouse brain microcirculation using a closed cranial window. J Vis Exp. 2010;4-7.

112. Ristagno G, Tang W, Sun S, Weil MH. Cerebral cortical microvascular flow during and following cardiopulmonary resuscitation after short duration of cardiac arrest. Resuscitation. 2008;77:229-34.

113. Ristagno G, Sun S, Tang W, Castillo C, Weil MH. Effects of epinephrine and vasopressin on cerebral microcirculatory flows during and after cardiopulmonary resuscitation. Crit Care Med. 2007;35:2145-9.

114. Ristagno G, Tang W, Huang L, et al. Epinephrine reduces cerebral perfusion during cardiopulmonary resuscitation. Crit Care Med. 2009;37:1408-15.

115. Kroppenstedt SN, Thomale UW, Griebenow M, et al. Effects of early and late intravenous norepinephrine infusion on cerebral perfusion, microcirculation, brain-tissue oxygenation, and edema formation in brain-injured rats. Crit Care Med. 2003;31:2211-21.

116. Pennings FA, Bouma GJ, Ince C. Direct observation of the human cerebral microcirculation during aneurysm surgery reveals increased arteriolar contractility. Stroke. 2004;35:1284-8.

117. Mathura KR, Bouma GJ, Ince C. Abnormal microcirculation in brain tumours during surgery. Lancet. 2001;358:1698-9.

118. Šitina $M$, Turek Z, Pařzková R, Černý V. In situ assessment of the brain microcirculation in mechanicallyventilated rabbits using sidestream dark-field (SDF) imaging. Physiol Res. 2011;60:75-81.

119. Sitina M, Turek Z, Parizkova R, Lehmann C, Cerny V. Preserved cerebral microcirculation in early stages of endotoxemia in mechanically-ventilated rabbits. Clin Hemorheol Microcirc. 2011;47:37-44.

120. Wan Z, Ristagno G, Sun S, Li Y, Weil MH, Tang W. Preserved cerebral microcirculation during cardiogenic shock. Crit Care Med. 2009;37:2333-7.

121. Pérez-Bárcena J, Goedhart P, Ibáñez J, et al. Direct observation of human microcirculation during decompressive craniectomy after stroke. Crit Care Med. 2011;39:1126-9.

122. Pérez-Bárcena J, Romay E, Llompart-Pou JA, et al. Direct observation during surgery shows preservation of cerebral microcirculation in patients with traumatic brain injury. J Neurol Sci. 2015;353:38-43. 



\section{Chapter 3 \\ 3}

Protocol for intraoperative assessment of the human cerebrovascular glycocalyx

R.H.L. Haeren, H. Vink, J. Staals, M.A.M.J. van Zandvoort, J. Dings, J.J. van Overbeeke,

G. Hoogland, K. Rijkers, O.E.M.G. Schijns 


\section{Abstract}

\section{Introduction}

Adequate functioning of the blood-brain barrier (BBB) is important for brain homeostasis and normal neuronal function. Disruption of the BBB has been described in several neurological diseases. Recent reports suggest that an increased permeability of the BBB also contributes to increased seizure susceptibility in epilepsy patients.

The endothelial glycocalyx is coating the luminal side of the endothelium and can be considered as the first barrier of the BBB. We hypothesize that an altered glycocalyx thickness plays a role in the etiology of temporal lobe epilepsy (TLE), the most common type of epilepsy. Here, we propose a protocol that allows intraoperative assessment of the cerebrovascular glycocalyx thickness in TLE patients and assess whether its thickness is decreased in TLE-patients when compared to controls.

\section{Methods and analysis}

This protocol is designed as a prospective observational case-control study in patients who undergo resective brain surgery as treatment for TLE. Control subjects are patients without a history of epileptic seizures, who undergo a craniotomy or burr hole surgery for other indications. Intraoperative glycocalyx thickness measurements of the sublingual, cortical, and hippocampal microcirculation are performed by videomicroscopy using sidestream darkfield imaging. Demographic details, seizure characteristics, epilepsy risk factors, intraoperative hemodynamic parameters, and histopathological evaluation are additionally recorded.

\section{Ethics and dissemination}

This protocol has been ethically approved by the local medical ethical committee (ID: NL51594.068.14) and complies with the Declaration of Helsinki and principles of Good Clinical Practice. Informed consent is obtained before study enrollment and only coded data will be stored in a secured database, enabling audit trail. Results will be submitted to international, peer-reviewed journals and presented at international conferences. 


\section{Introduction}

With a prevalence of 4-10 per 1000, epilepsy is one of the most common neurological disorders ${ }^{1}$. The most frequent type of focal epilepsy is temporal lobe epilepsy (TLE). Although most patients have adequate seizure control by using antiepileptic drugs, it is estimated that 22.5 - 30\% of patients are drug-resistant ${ }^{2,3}$. This implies that worldwide about 13 million patients suffer from drug-refractory epilepsy, which has a major impact, both medically and socioeconomically ${ }^{3,4}$. The development of new antiepileptic drugs has hardly reduced the number of drug-resistant patients, and therefore several different treatment alternatives have been explored over the past 25 years. Epilepsy surgery has been demonstrated to be a successful treatment alternative in selected patients. Furthermore, discovery of new drugs, targeted at specific underlying pathophysiological mechanisms keeps holding a promise for improved treatment of drug-resistant epilepsy patients.

In this regard, important discoveries on microvasculature abnormalities in TLE-patients have been reported, such as loss of blood-brain barrier (BBB) integrity ${ }^{5-7}$. BBB opening induces extravasation of proteins, like albumin, which in turn activates transforming growth factor $\beta$ (TGF $\beta$ ) signalling in astrocytes, leading to astrocytic transformation ${ }^{7,8}$. This transformation is characterized by downregulation of inward rectifier potassium channels and astrocytic glutamate transporters, leading to impaired cerebral homeostasis, altered neurovascular coupling, enhanced neuronal excitability, and upregulation of proinflammatory cytokines ${ }^{8,9}$. Moreover, leucocyte extravasation due to increased BBB permeability, contributes to neuronal excitability and reorganization of local neuronal networks ${ }^{9}$. Thus, loss of BBB integrity results in increased seizure susceptibility and contributes to epileptogenesis.

The barrier function of the BBB is mainly determined by the endothelium. Endothelial cells are interconnected by tight junctions and adherent junctions to prevent paracellular diffusion ${ }^{10}$. As a consequence, the endothelium forms a continuous cell membrane layer along the cerebral capillaries. Solutes and nutrients are transported by transport proteins expressed on the endothelial cells ${ }^{10,11}$. Endothelial cells thus restrict and actively control the passage of substances from the blood to the brain in order to tightly regulate cerebral homeostasis. The pericytes and astrocyte foot processes form a complex network surrounding the endothelial cells to induce and maintain endothelial barrier properties. As was recently pointed out, the endothelial glycocalyx could be a significant determinant of the BBB function as well ${ }^{12}$. The endothelial glycocalyx, further referred to as 'glycocalyx', is a gel-like layer lining the luminal surface of the endothelium. It has important barrier properties that reduce the interaction between endothelial cells and plasma cells and components. As a consequence, the glycocalyx limits leucocyte adhesion and protein extravasation ${ }^{13-16}$. The glycocalyx is a vulnerable layer that is easily disrupted resulting in a reduced thickness ${ }^{17}$. As part of the BBB, a disrupted glycocalyx would result in increased BBB permeability, and propagate leucocyte adhesion and extravasation. A number of 
laboratory and clinical studies have shown that both increased BBB permeability and leucocyte adhesion/extravasation play a role in epilepsy ${ }^{7,9,18}$.

So far, no data are available on glycocalyx thickness in epilepsy. The cerebrovascular glycocalyx has only been evaluated preclinically ${ }^{19-22}$. None of these studies have analyzed glycocalyx thickness in relation to neurological diseases. However, glycocalyx thickness can be assessed noninvasively as part of microcirculation imaging using sidestream darkfield (SDF) imaging ${ }^{12}$. To date, this technique has mainly been performed to assess the sublingual microcirculation.

Our hypothesis is that the cerebrovascular glycocalyx can be visualized clinically using SDF imaging, and that drug-resistant TLE-patients have a decreased cerebrovascular glycocalyx thickness in comparison to controls. In this paper, we present the study protocol according to the STROBE guidelines for case-control studies and SPIRIT protocol guidelines $^{23,24}$.

The primary objective of this case-control study is to visualize the cerebrovascular glycocalyx using SDF imaging in TLE-patients and control patients during brain surgery. Moreover, an eventual correlation between the cerebrovascular and sublingual glycocalyx dimensions is assessed.

\section{Methods}

\subsection{Study setting and population}

This is a multicenter prospective observational case-control study that involves assessment of the sublingual, cortical, and hippocampal glycocalyx. In conjunction, data are recorded regarding patient's medical history, current physiology, and cerebral pathology. Assessments take place during brain surgery, which is performed by the department of Neurosurgery of both the Maastricht University Medical Center+ (MUMC+) and the Zuyderland Medical Center (ZMC), respectively located in Maastricht and Heerlen, The Netherlands.

Patients with unilateral drug-resistant TLE that are designated for resective brain surgery, i.e. anterior temporal lobectomy and/or amygdalohippocampectomy, are possible study candidates. The diagnosis of unilateral TLE is based on thorough examination including a video-EEG, high-resolution MRI, neuropsychological examination and, when indicated, a PET- and or SPECT-scan, functional MRI, and eventually subdural or depth (stereo-EEG) electrode implantation. Subsequently, eligibility for resective surgery is assessed by a multidisciplinary team, consisting of neurologists, clinical neurophysiologists, neuropsychologists, neuroradiologists, and neurosurgeons.

The control group includes patients without a history of epileptic seizures, and who will undergo a craniotomy for intracranial tumor resection or neurovascular indications, like 
aneurysm clipping and arteriovenous malformation resection, or who will undergo burr hole surgery for tumor biopsy.

We have included an upper age limit of 60 years to avoid 'background' microvascular disease which is strongly age-related. Especially since tumor patients are already older, on average, than epilepsy patients, age could otherwise have been an important confounder. Control patients with vascular pathology, like aneurysms and arteriovenous malformation, are not known to have microcirculatory pathology. Moreover, cortical measurements are performed at a distance of the vascular pathology as allowed by the craniotomy, by example the superior temporal gyrus in a patient with an anterior cerebral artery aneurysm. Cortical measurements in the oncological control patients will also be performed at the furthest distance away of the abnormality possible, as allowed by the craniotomy.

\subsection{Eligibility criteria}

Inclusion criteria are: mentally competent adults between 18 and 60 years of age who will undergo resective surgery for unilateral drug-resistant TLE, or for a tumor or vascular abnormality, or burr hole surgery for tumor biopsy (Table 3.1). Exclusion from this study occurs in case of pregnancy, history of established hypertension, diabetes mellitus, hyperlipidemia, stroke or other cardiovascular disease, use of cardiovascular medication, or non-symptomatic signs of cerebral small vessel disease on brain MRI. Additionally, control patients in which no 'normal', 'non-compressed' and/or 'nonedematous' cerebral cortex can be assessed during surgery or in whom a history of seizures is reported, are excluded.

\begin{tabular}{ll}
\hline Inclusion criteria & \\
\hline TLE*-patients & Control-patients \\
$\begin{array}{l}\text { Mentally-competent patients between } 18 \text { and } 60 \\
\text { years of age. }\end{array}$ & $\begin{array}{l}\text { Mentally-competent patients between } 18 \text { and } 60 \\
\text { years of age. }\end{array}$ \\
$\begin{array}{l}\text { Patients undergoing resective surgery as treatment } \\
\text { of drug-resistant TLE with an epileptic focus that is }\end{array}$ & $\begin{array}{l}\text { Patients undergoing an elective craniotomy for } \\
\text { intracranial tumor resection or neurovascular surgery, } \\
\text { located in a non-eloquent area. }\end{array}$ \\
& $\begin{array}{l}\text { or undergoing burr hole surgery for tumor biopsy in a } \\
\text { non-eloquent area. }\end{array}$ \\
\hline
\end{tabular}

Exclusion criteria
Applicable to all patients
Patients who are pregnant, who have been diagnosed with diabetes mellitus, hyperlipidemia, stroke, or
other cardiovascular diseases, or use of cardiovascular medication.
Patients who, based on brain MRI**, show non-symptomatic signs of cerebral small vessel disease.
Applicable to control-patients only
Patients in whom no 'normal', 'non-compressed' and/or 'non-edematous' cerebral cortex can be assessed
intraoperatively.
Patients with a history of seizures.

Table 3.1 Overview of inclusion and exclusion criteria.

* TLE: temporal lobe epilepsy; ${ }^{* *}$ MRI: magnetic resonance imaging 


\subsection{Intervention protocol}

The glycocalyx thickness is assessed intraoperatively at the following time points: 1 . a sublingual measurement (M1) directly following the induction of general anesthesia, 2. a cortical measurement (M2) upon opening of the meninges allowing a direct view at the cortex and 3. a hippocampal measurement (M3) only in TLE-patients. The latter measurement is performed upon removal of the temporal neocortex allowing direct view at the hippocampus. At each time point, systolic and diastolic blood pressure, heart rate, pulse oxygen saturation, hemoglobin concentration, and hematocrit are additionally recorded.

Glycocalyx measurements are performed using a SDF video microscope. The camera consists of a central light guide with magnifying lens and concentric light emitting diodes. The diodes emit light at a wavelength of $530 \mathrm{~nm}$, which is absorbed by (de)oxyhemoglobin in erythrocytes. Consequently, erythrocytes appear black on a grayish background. This technique has mainly been performed on the sublingual microcirculation but has previously been used for cerebral microcirculation assessment

as well ${ }^{25-29}$. However, the glycocalyx was not measured in these studies ${ }^{25-28}$. Recently, it was pointed out that SDF imaging, when combined with dedicated software, is the most suitable technique for clinical cerebrovascular glycocalyx visualization ${ }^{12}$.

In order to reduce user-induced variations, image acquisition is trained. Using the camera with low pressure, minimizing movement artefacts and optimizing light intensity and focus are of particular importance to further reduce variability.

The glycocalyx analysis is based on the principle of the erythrocyte-endothelial exclusion zone. Since erythrocytes do not significantly compress the glycocalyx, there is an exclusion zone between the red blood cell column (RBCC) and the endothelium. GlycoCheck (C) software automatically selects approximately 3,000 microcirculatory vessel segments and measures the RBCC width at each segment. Based on the RBCC width distribution, the total perfused diameter (Dperf) is assessed by linear regression analysis of the $25^{\text {th }}$ and $75^{\text {th }}$ RBCC width percentiles ${ }^{12}$. The perfused boundary region (PBR) is the outermost luminal part of the glycocalyx that is only slightly permeable for erythrocytes. This region is calculated in a two-dimensional plane as follows: $\mathrm{PBR}=$ (Dperf - median RBCC)/2. As an unstable or damaged glycocalyx is more accessible to erythrocytes, an increased RBCC and, consequently, increased Dperf and PBR values signify a damaged glycocalyx ${ }^{29}$.

\subsection{Outcome}

The primary outcome of this study is cortical glycocalyx thickness in TLE patients and controls. The thickness is estimated by measuring the perfused boundary region (PBR) and the total perfused diameter (Dperf), both expressed in micrometers $(\mu \mathrm{m})$.

As secondary outcome, sublingual, cortical, and hippocampal glycocalyx thickness in TLE-patients and controls are evaluated within and between groups. In addition, we aim to analyze the relation between these measures and epilepsy-specific risk factors 
(e.g. febrile seizures, traumatic brain injury), seizure characteristics, drug use (e.g. type of drugs, dosage), and histopathological outcome (e.g. degree of hippocampal sclerosis, type of cortical dysplasia). Finally, glycocalyx thickness values will be correlated to general demographic (e.g. age, gender, bodyweight, smoking status) and intraoperative clinical parameters.

\subsection{Recruitment capacity, consent and timeline}

We have calculated a sample size of 15 patients for each group, to be included over 4 years. At the MUMC+ and ZMC, all types of surgery required for inclusion are performed at least 15-20 times every year. Based on these surgery numbers, the likelihood to recruit the calculated sample size is high.

The study participants' timeline is outlined below, and an overview is also given in Figure 3.1.

\section{Recruitment}

Patients will visit the neurosurgical outpatient clinic preoperatively. During this visit, the neurosurgeon will ask the patients whether they are interested to take part in the study. If interested, they will receive the applicable patient information brochure (PIB). Within a few days, possible candidates will be informed more extensively by one of the investigators about the study objective, procedures, benefits, risks, and insurance. Participation decisions will be inquired at the day of admission to the hospital. Patients are allowed at least 3 days to consider participation for the study, starting from the moment of receiving the PIB. Participation is voluntarily and does not affect standard treatment in any way. Patients will be informed that they can decide to end their participation in the study at any time.

\section{Informed consent}

At the day of hospital admission, patient's study participation is ultimately inquired. When patients agree on participation, the informed consent agreement will be signed in duplicate.

\section{Data recording}

Following informed consent agreement, patient data will be recorded from the digital patient files. The digital patient files encompass the digital patient dossier at the MUMC+, ZMC and, when applicable, the file of the multidisciplinary epilepsy surgery work-up.

\section{Intraoperative measurements}

A total of two or three glycocalyx measurements will be performed, as described in paragraph 2.3. All measurements are performed at the operating theatre when the patient is under general anesthesia. The hippocampal measurement is solely performed in TLE-patients. 


\section{End of study}

Directly following the final measurements, i.e. M2 in control-patients and M3 in TLEpatients, patients have reached the endpoint of this study.

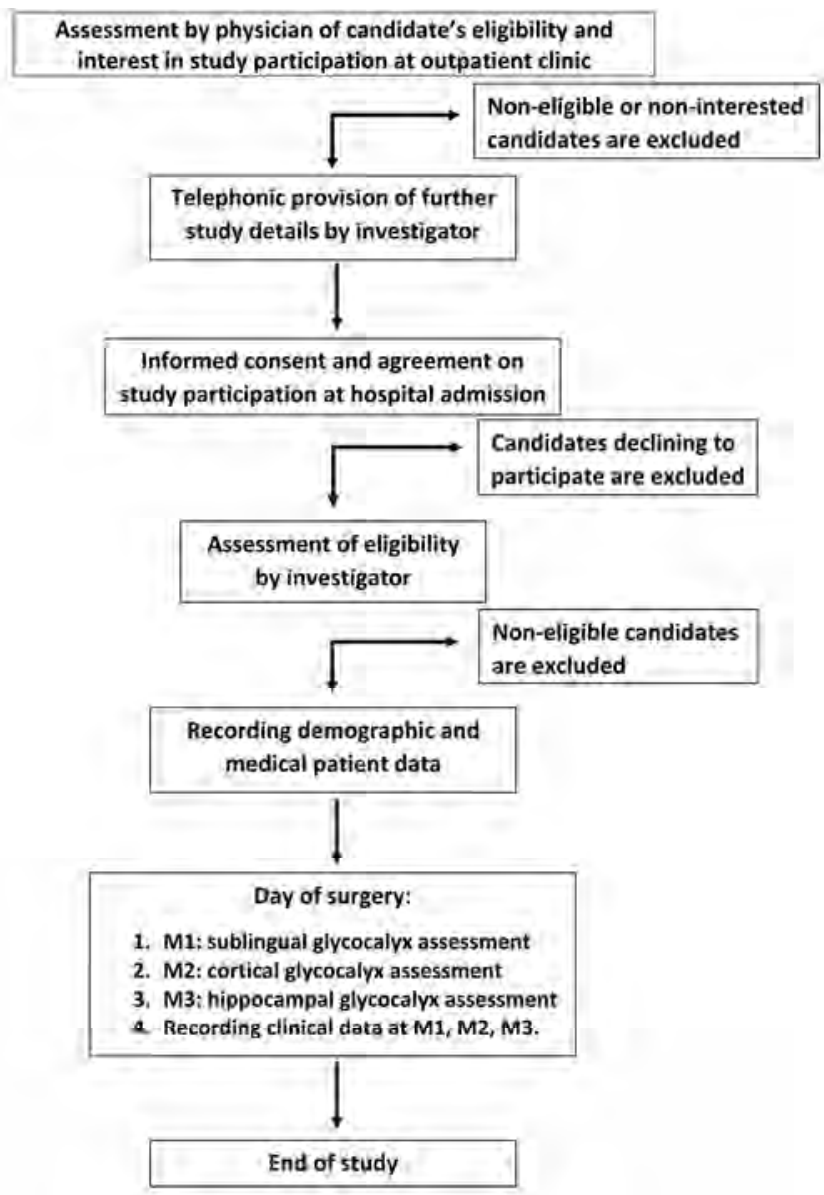

Figure 3.1 Timeline of informed consent, inclusion, and study procedures of study candidates.

\subsection{Population size}

We have calculated a population size of 15 patients per group. This number is based on a power of $80 \%$ to detect a difference of cortical glycocalyx thickness of at least $12 \%$ between groups with a standard deviation of $15 \%$, at a significance level of 0.05 and an expected drop-out of 2 patients.

Since there is no literature on cerebrovascular glycocalyx thickness, we have reviewed and assessed the literature on variation in sublingual glycocalyx measurements. Intra- 
individual variation of sublingual glycocalyx thickness, assessed using SDF imaging, has been found to be $\pm 5 \%{ }^{30}$. A difference in PBR thickness of $9.6-12.5 \%$ with a standard deviation of $\pm 15 \%$ has been found relevant when comparing a disease state to healthy controls $^{29-32}$. Based on these studies, we have determined minimal glycocalyx difference and standard deviation. Due to the explorative nature of our study, clinical relevance of a $12 \%$ difference is indistinct.

\subsection{Data processing}

\subsubsection{Procedures}

This study complies with the Declaration of Helsinki, and will be conducted in accordance with the principles of Good Clinical Practice (GCP). Standardized processing files for obtaining informed consent, measurement procedures, reporting (serious) adverse events and recording patient and measurement data parameters in the electronic case report file (eCRF), are available. Investigators obtaining informed consent from the patient, performing glycocalyx measurements, and recording eCRF data will receive specific training beforehand.

\subsubsection{Data management}

Patient's demographic and clinical data are recorded in an eCRF at a secure encrypted database (by Castor $\mathrm{EDC}^{\odot}$ ), which enables audit trail and is GCP certified. Measurement procedures are trained and standardized as described in paragraph 2.3. Measurement data is collected at a secure encrypted laptop and outcome is recorded in the eCRF. After verification of recorded data to source data by one the executive investigators, recorded data in the eCRF by Castor EDC $^{\odot}$ will be exported to a SPSS file for further statistical analysis.

Patients will be assigned an alphanumeric sequential study number to identify all clinical data. Patient's demographic data linked to the assigned study number is documented in a separate screening database held on a secure computer at both study sites. Source data, the code encrypting document, and coded data in the study database are locked and only accessible to the principal and executive researchers, and monitors. On completion of the study, the study database will be locked and data is securely archived for 15 years in accordance with local policy.

Due to the nature and short participation time period of this study, we expect full patient retention and adherence.

\subsection{Safety}

The principal investigator (OS) has overall responsibility for the execution of this study including safety. Individual investigators will be responsible for reporting adverse events (AEs) and serious adverse events (SAEs) to the principal investigator. SAEs are 
defined as AEs resulting in death, life threatening events, prolonged hospital stays, or significant disability. There are no reported (S)AEs associated with the use of SDF imaging in the current literature. A possible attributable risk of glycocalyx measurement is sublingual, cortical, or hippocampal contusion due to pressure on the tissue during the measurement. We deem risk frequency and severity as low. Moreover, cortical or hippocampal local contusion will take place in non-eloquent and to-be-resected tissue. Postoperative consequences due to this contusion are unlikely. All events are reviewed by the principal investigator to decide if there is a causal link and, when applicable, appropriate action will be undertaken. SAEs will be reported to the local medical ethical committee (METC azM/UM) according to local policy. Liability and subject insurance is provided.

\subsection{Statistical analysis}

Data are presented as mean and standard deviation when normally distributed, and non-normally distributed data as median and interquartile range. Glycocalyx dimensions between groups are compared using the independent T-test or the MannWhitney test, as appropriate. Correlation between sublingual and cortical, sublingual/cortical and hippocampal glycocalyx thickness is calculated by Pearson's or Spearman's correlation coefficient, when data is normally or non-normally distributed, respectively. In TLE-patients, the cortical and hippocampal glycocalyx thickness results are correlated to seizure characteristics, epilepsy risk factors, anti-epileptic drug usage, and histological findings of pathological examination by using Pearson's or Spearman's correlation coefficient or uni- and multivariate regression analysis, as appropriate. Demographic and clinical parameters are correlated to glycocalyx thickness results by using uni- and multivariate regression analysis. A p-value of $<0.05$ will be considered statistically significant. Statistical analysis is performed using SPSS software.

Interim-analyses will be performed yearly and following data recording of 5 patients in each group. When a significant difference between the groups regarding the primary outcome is found, the study will be terminated prior to inclusion of 15 patients in each group. Interim-analyses are reported to the local medical ethical committee.

\subsection{Monitoring and auditing}

This study was classified as minimal-risk by the local data monitoring committee (CTCM). Monitoring visits include review of consent and study procedures according to study protocols, source data and audit trail verification, and the review of (serious) adverse event reporting. Monitoring is independent and performed at least once a year. Monitor evaluations are reported to the local medical ethical committee. Unannounced audits can be performed by the audit team of the CTCM. 


\subsection{Ethics, amendments, and dissemination}

This research protocol has been approved by the local medical ethical committee (METC azM/UM) and has been assigned the following protocol ID: NL51594.068.14. Also, this study has been registered at the Netherlands National Trial Register (ID: NTR 5568). The NTR is acknowledged by the WHO and International Committee of Medical Journal Editors (ICMJE).

Substantive protocol amendments will be reported, reviewed, and approved by the METC azM/UM before application. In the currently presented protocol, a variety of substantive protocol amendments have already been incorporated. Substantive amendments were introduced due to lagging inclusion. The substantive amendments included extending the study by the addition of the ZMC as a study center, enlarging the control-group with burr hole tumor biopsy and neurovascular surgery patients, and reducing sublingual measurements from three to one.

Results of this study will be evaluated yearly and following 5 participants in both groups. Results will be recorded using audit trails to increase reproducibility. Study protocol and results will be submitted to peer-reviewed journals and presented at international conferences.

\section{Discussion}

Microvascular injury, in particular increased permeability of the wall of the cerebral microcirculation, seems to play a role in the transformation of astrocytes and increased seizure susceptibility in epilepsy. It is hypothesized that leakage of albumin orchestrates this transformation leading to a disturbed potassium and glutamate metabolism and extracellular cerebral homeostasis ${ }^{7,8}$. Upregulation of proinflammatory cytokines as a response to leakage of leucocytes further contributes to the subsequent increased neuronal excitability 9 . However, it is still unclear why some patients do and some patients do not develop epilepsy following a first seizure. Heinemann et al. have brought up the hypothesis that lasting dysfunction of the cerebral microcirculation results in dysregulation of the normal network response to injury, leading to the development of epilepsy. Therefore, they have emphasized the urgent need for noninvasive clinical visualization of the cerebral microcirculation in order to evaluate local dynamical functioning and possible injury ${ }^{8}$.

The glycocalyx is a significant component of the cerebral microcirculation and involved in the regulation of plasma cell adhesion, oxidative stress, and shear stress reduction ${ }^{12,13}$. The glycocalyx is easily disrupted, i.e. reduced in thickness ${ }^{17}$. Disruption is induced by local cytokine expression and ischemia, and results in inflammation, edema, oxidative stress and loss of vascular responsiveness ${ }^{12}$. Also, many vascular disease risk factors and specific diseases like lacunar stroke, sepsis, and renal failure are associated with decreased glycocalyx thickness ${ }^{29,33-36}$. 
The glycocalyx plays an important role in vascular wall permeability. Albumin extravasation is prohibited by the negatively charged glycocalyx. Moreover, leukocytes cannot attach to endothelial surface components including important cell-adhesion molecules, like ICAM-1 and VCAM-1, that reside within the glycocalyx. As a consequence, leucocyte adhesion and extravasation is limited by a healthy glycocalyx. Therefore, a disturbed glycocalyx is suspected to play a significant role in increased BBB permeability including albumin and leucocyte extravasation, as is seen in epilepsy.

Assessment of the cerebrovascular glycocalyx offers the opportunity to gain greater insights in its thickness and function at the level of the cerebral microcirculation. Advances in microcirculation imaging by handheld SDF-based video microscopes enables real-time clinical assessment of the microcirculation. Several cerebral microcirculation parameters, but not the glycocalyx, have been evaluated using this technique ${ }^{25-28}$. It was recently pointed out that SDF imaging is the most eligible technique to assess the cerebrovascular glycocalyx, when proper software is subjoined $^{12}$. Using SDF imaging, we aim to visualize the cerebrovascular glycocalyx and measure its thickness in TLE-patients and controls. Subsequently, cerebrovascular glycocalyx dimensions are compared to sublingual dimensions to establish whether glycocalyx dimensions are regulated at a systemic level.

In this study, we will determine whether glycocalyx thickness is reduced in TLE-patients when compared to controls. Included TLE-patients have undergone thorough examination and were selected by a multidisciplinary team. Naturally, genuinely healthy controls for intracranial cerebrovascular glycocalyx assessment are not available, making the selected patients the most suitable candidates as controls. Since an intact hemodynamic circulation is required for SDF-imaging, post-mortem patients cannot be included as controls.

The upper age cut-off of 60 years is included to reduce the influence of microcirculatory degeneration which is associated to increasing age. As tumor patients are, on average, older patients than epilepsy patients, this could otherwise have been an important confounder. In order to limit the risk of vascular and hemodynamic abnormalities of the visualized and assessed vessels, patients will only be included as controls when noncompressed and/or non-edematous cerebral cortex can be assessed during surgery. In the control group, the cortical measurements are performed at the furthest distance away of the abnormality, as is allowed by the craniotomy.

Typical pathological findings in TLE include hippocampal sclerosis characterized by neuronal cell loss, reactive astrogliosis, mossy fiber sprouting, and granular cell dispersion ${ }^{5}$. Unfortunately, assessment of the hippocampal glycocalyx can only be performed in TLE-patients, as the hippocampus will rarely be exposed in the control subjects. Hippocampal glycocalyx thickness of TLE-patients will therefore be compared to cortical glycocalyx thickness and cannot be compared to hippocampal controls. 
There are some important variables that could affect the glycocalyx thickness that are not directly assessed in this study. These variables concern hemodynamic variations, intravascular volume variations and the effect of different anesthetics. Due to the explorative nature of this study, we have not included these variables, although posthoc analyses will be performed to determine confounding. Another reason for exclusion of these variables is that this would necessitate additional preoperative (awake) and intraoperative sublingual measurements of the glycocalyx. At first, these measurements were included in our study protocol. But due to anxiety for the preoperative measurement, and the risks for sterility of the surgery due to the intraoperative measurements, these sublingual measurements were excluded in an amendment (see paragraph 2.11). The possible effects of intraoperative hemodynamic variation and anesthesia on glycocalyx thickness could however be an interesting future study.

When clinically relevant differences in glycocalyx dimensions are found, future research to determine glycocalyx component variation, glycocalyx permeability determinants and mechanisms of disruption and repair, is required. Subsequently, repair of the glycocalyx could be a selective and efficient, yet hypothetical, target for modification of increased BBB permeability. This would open a new field of pharmacological interventions for currently drug-resistant epilepsy patients. 


\section{References}

1. Forsgren L, Beghi E, Õun A, Sillanpää M. The epidemiology of epilepsy in Europe - A systematic review. Eur J Neurol. 2005;12:245-53.

2. Picot $M C$, Baldy-Moulinier $M$, Daurès JP, Dujols $P$, Crespel $A$. The prevalence of epilepsy and pharmacoresistant epilepsy in adults: A population-based study in a Western European country. Epilepsia. 2008;49:1230-8.

3. Laxer KD, Trinka E, Hirsch $\mathrm{J}$, et al. The consequences of refractory epilepsy and its treatment. Epilepsy Behav. 2014;37:59-70.

4. Jennum P, Gyllenborg J, Kjellberg J. The social and economic consequences of epilepsy: A controlled national study. Epilepsia. 2011;52:949-56.

5. Thom M. Review: Hippocampal sclerosis in epilepsy: A neuropathology review. Neuropathol. Appl. Neurobiol. 2014;40:520-43.

6. Mott RT, Thore CR, Moody DM, Glazier SS, Ellis TL, Brown WR. Reduced ratio of afferent to total vascular density in mesial temporal sclerosis. J Neuropathol Exp Neurol. 2009;68:1147-54.

7. van Vliet EA, Aronica E, Gorter JA. Role of blood-brain barrier in temporal lobe epilepsy and pharmacoresistance. Neuroscience. 2014;277:455-73.

8. Heinemann U, Kaufer D, Friedman A. Blood-brain barrier dysfunction, TGF $\beta$ signaling, and astrocyte dysfunction in epilepsy. Glia. 2012;60:1251-7.

9. Vezzani A, Friedman A, Dingledine RJ. The role of inflammation in epileptogenesis. Neuropharmacology. 2013;69:16-24.

10. Abbott NJ, Patabendige AAK, Dolman DEM, Yusof SR, Begley DJ. Structure and function of the bloodbrain barrier. Neurobiol Dis. 2010;37:13-25.

11. Ballabh P, Braun A, Nedergaard M. The blood-brain barrier: an overview. Neurobiol Dis. 2004;16:1-13.

12. Haeren RHL, van de Ven SEM, van Zandvoort MAMJ, et al. Assessment and imaging of the cerebrovascular glycocalyx. Curr Neurovasc Res. 2016;13(3):249-60.

13. Reitsma S, Slaaf DW, Vink H, Van Zandvoort MAMJ, Oude Egbrink MGA. The endothelial glycocalyx: Composition, functions, and visualization. Pflugers Arch. 2007;454:345-59.

14. Curry FE, Adamson RH. Endothelial glycocalyx: Permeability barrier and mechanosensor. Ann. Biomed. Eng. 2012;40:828-39.

15. Constantinescu AA, Vink H, Spaan JE. Endothelial cell glycocalyx modulates immobilization of leukocytes at the endothelial surface. Arterioscler Thromb Vasc Biol. 2003;23:1541-7.

16. Rops $A L$, van den Hoven MJ, Baselmans MM, et al. Heparan sulfate domains on cultured activated glomerular endothelial cells mediate leukocyte trafficking. Kidney Int. 2008;73:52-62.

17. Reitsma S, Oude Egbrink MGA, Vink H, et al. Endothelial glycocalyx structure in the intact carotid artery: A two-photon laser scanning microscopy study. J Vasc Res. 2011;48:297-306.

18. Friedman A. Blood-brain barrier dysfunction, status epilepticus, seizures, and epilepsy: a puzzle of a chicken and egg? Epilepsia. 2011;52 Suppl 8:19-20.

19. Mazzetti S, Librizzi L, Frigerio S, de Curtis M, Vitellaro-Zuccarello L. Molecular anatomy of the cerebral microvessels in the isolated guinea-pig brain. Brain Res. 2004;999:81-90.

20. Hempel C, Hyttel P, Kurtzhals J Al. Endothelial glycocalyx on brain endothelial cells is lost in experimental cerebral malaria. J Cereb Blood Flow Metab. 2014;34:1107-10.

21. Sillesen M, Rasmussen LS, Jin G, et al. Assessment of coagulopathy, endothelial injury, and inflammation after traumatic brain injury and hemorrhage in a porcine model. J Trauma Acute Care Surg. 2014;76:12-9.

22. Jepsen $\mathrm{CH}$, DeMoya MA, Perner A, et al. Effect of valproic acid and injury on lesion size and endothelial glycocalyx shedding in a rodent model of isolated traumatic brain injury. J Trauma Acute Care Surg. 2014;77:292-7.

23. von Elm E, Altman DG, Egger M, Pocock SJ, Gøtzsche PC, Vandenbroucke JP. The strengthening the reporting of observational studies in epidemiology (STROBE) statement: explanation and elaboration. J Clin Epidemiol. 2008;61:344-9.

24. Chan AW, Tetzlaff JM, Altman DG, et al. SPIRIT 2013 statement: Defining standard protocol items for clinical trials. Ann Intern Med. 2013;158:200-7. 
25. Pérez-Bárcena J, Goedhart P, Ibáñez J, et al. Direct observation of human microcirculation during decompressive craniectomy after stroke. Crit Care Med. 2011;39:1126-9.

26. Pérez-Bárcena J, Romay E, Llompart-Pou JA, et al. Direct observation during surgery shows preservation of cerebral microcirculation in patients with traumatic brain injury. J Neurol Sci. 2015;353:38-43.

27. Wan Z, Ristagno G, Sun S, Li Y, Weil MH, Tang W. Preserved cerebral microcirculation during cardiogenic shock. Crit Care Med. 2009;37:2333-7.

28. Šitina M, Turek Z, Pařzková R, Černý V. In situ assessment of the brain microcirculation in mechanicallyventilated rabbits using sidestream dark-field (SDF) imaging. Physiol Res. 2011;60:75-81.

29. Martens RJH, Vink H, Van Oostenbrugge RJ, Staals J. Sublingual microvascular glycocalyx dimensions in lacunar stroke patients. Cerebrovasc Dis. 2013;35:451-4.

30. Nieuwdorp M, Meuwese MC, Mooij HL, et al. Measuring endothelial glycocalyx dimensions in humans: a potential novel tool to monitor vascular vulnerability. J Appl Physiol. 2008;104:845-52.

31. Snoeijs MG, Vink H, Voesten N, et al. Acute ischemic injury to the renal microvasculature in human kidney transplantation. Am J Physiol Renal Physiol. 2010;299:F1134-40.

32. Dane MJC, Khairoun MR, Hyun Lee $D$, et al. Association of kidney function with changes in the endothelial surface layer. Clin J Am Soc Nephrol. 2014;9:698-704.

33. Vlahu CA, Lemkes BA, Struijk DG, Koopman MG, Krediet RT, Vink H. Damage of the endothelial glycocalyx in dialysis patients. J Am Soc Nephrol. 2012;23:1900-8.

34. Becker BF, Chappell D, Jacob M. Endothelial glycocalyx and coronary vascular permeability: The fringe benefit. Basic Res Cardiol. 2010;105:687-701.

35. Ostrowski SR, Berg RMG, Windeløv NA, et al. Coagulopathy, catecholamines, and biomarkers of endothelial damage in experimental human endotoxemia and in patients with severe sepsis: $A$ prospective study. J Crit Care. 2013;28:586-96.

36. Singh A, Fridén V, Dasgupta I, et al. High glucose causes dysfunction of the human glomerular endothelial glycocalyx. Am J Physiol Renal Physiol. 2011;300:F40-8. 



\section{Chapter 4}

In vivo imaging of the human cerebral

microcirculation and its glycocalyx: a feasibility study

R.H.L. Haeren, K. Rijkers, O.E.M.G. Schijns, J. Dings, G. Hoogland, M.A.M.J. van Zandvoort, H. Vink, J.J. van Overbeeke Adapted from the publication in Journal of Neuroscience Methods, 2018 


\section{Abstract}

\section{Introduction}

The cerebral microcirculation and its glycocalyx, a matrix coating the luminal endothelium, are key regulators of blood-brain barrier permeability and cerebral blood flow. Microvascular abnormalities are described in several neurological disorders. However, assessment of the cerebral microcirculation and glycocalyx has mainly been performed ex vivo.

\section{New method}

Here, the technical feasibility of in vivo assessment of the human cerebral microcirculation and its glycocalyx using sidestream dark field (SDF) imaging is discussed.

Using SDF imaging, the sublingual (reference), cortical, and hippocampal microcirculation and glycocalyx were evaluated in patients who underwent resective brain surgery as treatment for drug-resistant temporal lobe epilepsy. Subsequently, vessel density, and the perfused boundary

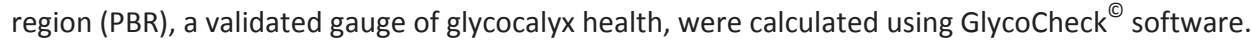

\section{Results}

A total of five patients were included. The sterile cerebral measurements were more difficult to obtain than the sublingual. This was probably due to the introduction of a sterile slipcover. Results on vessel density and PBR showed similar patterns at all three measurement sites.

\section{Comparison with existing methods}

This is the first report on in vivo assessment of the human cerebrovascular glycocalyx. Assessment of this glycocalyx is an additional application of in vivo imaging of the cerebral microcirculation using SDF imaging. This method enables functional analysis of the microcirculation and glycocalyx.

\section{Conclusions}

SDF imaging is a safe, quick, and straightforward technique to evaluate the cerebral microcirculation and glycocalyx. Because of their eminent role in cerebral homeostasis, this method may significantly advance research on the pathophysiology of various neurological disorders. 


\section{Introduction}

In recent years, the study on the pathophysiology of neurological disorders has shifted from a traditional neuronal focus towards a more integrative paradigm that emphasizes cell-cell signaling ${ }^{1}$. In particular, the interactions between the vascular and neuronal tissue have been of interest ${ }^{2-6}$. Physiological brain function is highly dependent on adequate structure and function of the cerebral microcirculation due to the lack of energy reserves, an on average high and rapidly changing metabolic rate, and tight regulation of brain homeostasis ${ }^{3,6,7}$. Hence, interest in the cerebral microcirculation and the neurovascular interface rapidly increases.

The microcirculation regulates local blood flow to meet neuronal metabolic demands, a mechanism known as neurovascular coupling ${ }^{3,7,8}$. Besides this, it orchestrates the rate of delivery and exchange of vital nutrients across the blood-brain barrier (BBB $)^{3,9-11}$.

The BBB exerts its barrier function mainly by endothelial cells which are interconnected by tight junctions, and surrounded by the basal lamina, pericytes and astrocytes forming a stabilizing network ${ }^{3}$. Another significant barrier function may be exerted by the endothelial glycocalyx, further referred to as 'glycocalyx' ${ }^{12}$. This gel-like matrix covers the luminal side of the endothelium. An intact glycocalyx reduces interaction between the endothelium and plasma components, and plays an important role in flow regulation as a mechanotransductor ${ }^{13,14}$. Consequently, the glycocalyx is a key regulator of vascular permeability, cerebral blood flow, capillary perfusion, and cell adhesion $^{12,14,15}$. Preservation of an intact glycocalyx is an important aspect of vascular health. Not surprisingly, many vascular diseases and related risk factors like stroke, renal failure, sepsis, hypertension, and diabetes mellitus are associated with a disturbed glycocalyx ${ }^{16-21}$.

Imaging and assessment of the human cerebral microcirculation and its glycocalyx has mainly been performed ex vivo by microscopic evaluation of structural changes and analysis of vascular (wall) stainings and markers ${ }^{22-26}$. However, functional analysis of the microcirculation cannot be performed ex vivo. Nowadays, non-invasive imaging techniques, including sidestream dark field (SDF) imaging, allow in vivo assessment of the microcirculation and glycocalyx. SDF imaging has mainly been performed to assess the sublingual microcirculation ${ }^{16,17,27}$. Recently, SDF imaging and its technical predecessor have also been used to assess the cerebral microcirculation (Mathura et al. 2001; Uhl et al. 2003; Pennings et al. 2004, 2006, 2009; Wan et al. 2009; Pérez-Bárcena et al. 2011, 2015). However, results were limited to specific case series. Moreover, in vivo assessment of the cerebrovascular glycocalyx has not been performed to date. The addition of specific software to SDF imaging is suggested to enable glycocalyx assessment $^{12}$.

We have developed a prospective observational case-control study in which the human sublingual, cortical, and hippocampal microcirculation and glycocalyx are assessed in vivo by SDF imaging. Microvascular abnormalities and BBB dysfunction have previously 
been associated with the pathophysiology of epilepsy ${ }^{24,25,36-39}$. Therefore, the intention of this study was to evaluate cerebral microvascular and glycocalyx properties in epilepsy patients. In this paper, the technical feasibility of in vivo, i.e. during surgery, assessment of the cerebral microcirculation and its glycocalyx in epilepsy patients is discussed.

\section{Materials and methods}

This study was approved by the local medical ethical committee (METC azM/UM) and complies with the Declaration of Helsinki and principles of Good Clinical Practice. This study has been registered at The Netherlands National Trial Register (ID: NTR 5568). The study protocol was published recently ${ }^{40}$. Patients were included upon obtained informed consent.

\subsection{Participants}

Included epilepsy patients were mentally competent patients between 18 and 60 years of age who were candidates for epilepsy surgery because of chronic drug-resistant temporal lobe epilepsy (TLE) as was revealed by thorough preoperative examination. Surgical treatment included an anterior temporal lobectomy plus amygdalohippocampectomy. Exclusion criteria were pregnancy, history of hypertension, diabetes mellitus, hyperlipidemia, stroke or other cardiovascular diseases, use of cardiovascular medication, or signs of cerebral small vessel disease on cerebral MRI.

\subsection{Imaging procedures: videomicroscopy}

Measurements were performed using a Capiscope video-microscope camera (KK Technology; Honiton, UK). This handheld camera is based on SDF technique. The camera contains a central light guide that is surrounded by concentrically placed light emitting diodes (LEDs) emitting light at a wavelength of $530 \mathrm{~nm}$, which is absorbed by (de)-oxyhemoglobin in erythrocytes. Consequently, erythrocytes appear as dark disks on a grayish background. The light is re-emitted by the central light guide towards the objective lens providing an image of the illuminated area. Images of $720 \times 526$ pixels are collected with a $5 x$ magnification, at 23 frames per second. To reduce operatorrelated variability, all images were acquired by the same investigator $(\mathrm{RH})$.

\subsection{Imaging procedure protocol}

Three intraoperative measurements were performed: a sublingual measurement (M1) directly following the induction of general anesthesia, a cortical measurement (M2), and a hippocampal measurement (M3). The sublingual measurement is included as a 
validated reference, as most reports on microcirculation assessment using SDF imaging are performed sublingually. When performing the cerebral measurements, the SDF camera is enclosed within a sterile slipcover (Camera Drape with EZ SERT, Microtek Medical Inc). At each time point, systolic and diastolic blood pressure, and heart rate were recorded.

For each measurement, at least 10 sites and a minimum of 3000 microvessel segments were selected automatically. This selection takes place when focus, contrast, and image quality are within predefined acceptable ranges. Of these, obtaining adequate focus is performed manually. Pressure artefacts were avoided, and artefacts by blood clots were reduced by rinsing the cerebral tissue before measurement using $37^{\circ} \mathrm{C}$ Ringer's lactate.

GlycoCheck (C) software was used to select and measure the width of the red blood cell column (RBCC) in vessel segments with median RBCC widths ranking from 5-20 $\mu \mathrm{m}$. Determination of the vessel diameter is difficult in these small vessels since only the erythrocytes are visualized, instead of the vessel wall. As the reported diameters correspond to the median RBCC diameter of a certain vessel, they are an underestimation of actual anatomic vessel diameter.

\subsection{Outcome parameters}

Functional vessel density for each RBCC diameter was automatically determined by the Glycocheck (C) software and was expressed as vessels per $\mathrm{mm}^{2}$.

The glycocalyx is also evaluated using Glycocheck (c) software. Since compression of a 'healthy' glycocalyx by erythrocytes is limited, an exclusion zone between the RBCC and endothelium is apparent. This exclusion zone forms the basis of glycocalyx analysis. The total perfused diameter (Dperf) is determined by linear regression analysis of the $25^{\text {th }}$ and $75^{\text {th }}$ RBCC width percentiles ${ }^{12}$. The perfused boundary region (PBR) is the outermost luminal part of the glycocalyx that is slightly permeable for erythrocytes. The PBR, expressed in micrometers $(\mu \mathrm{m})$, is an indirect gauge to measure glycocalyx stability, and in a two-dimensional plane is calculated as follows: PBR = (Dperf - median $\mathrm{RBCC} / 2$. An unstable or damaged glycocalyx is more accessible for erythrocytes resulting in an increased $\mathrm{RBCC}$ width and subsequent increases of Dperf and PBR values.

\subsection{Data and statistical analysis}

All relevant clinical and measurement data were recorded in a secured and anonymized database. Clinical and procedural parameters are presented as mean \pm standard error of mean (SEM). Results of vessel density and PBR are presented as mean \pm SEM and are illustrated in graphs per RBCC diameter. The predictive value of the sublingual PBR for cortical and hippocampal PBR is calculated using Pearson correlation coefficient. Calculations were performed using SPSS (IBM, SPSS Statistics for Windows, version 20.0, 2011). 


\section{Results}

\subsection{General characteristics}

A total of five TLE-patients with a mean age of 44.4 years were included in this study. Further clinical details are summarized in Table 4.1. All measurements were performed after an overnight fast which was required for anesthesia induction. Assessment of the sublingual and hippocampal microcirculation was completed in all patients, while the cortical measurements were accomplished in 4 TLE-patients.

Hemodynamic parameters for each measurement are presented in Table 4.2. In this table, the duration of each assessment is also provided. Mean time for sublingual microcirculation (M1) assessment was $40.0 \pm 6.3$ seconds. The duration of the cortical (M2) and hippocampal (M3) measurements was substantially longer, up to almost 6 and 4 minutes, respectively. The number of recordings to accomplish at least 3000 measurement sites was markedly higher in $\mathrm{M} 2$ and $\mathrm{M} 3$.

\begin{tabular}{ccccccccccc}
\hline & Sex & $\begin{array}{c}\text { Age } \\
\text { (years) }\end{array}$ & $\begin{array}{c}\text { BMI } \\
\left(\mathrm{kg} / \mathrm{m}^{2}\right)\end{array}$ & Smok & Indication & Side & $\begin{array}{c}\text { Cortical } \\
\text { site }\end{array}$ & PA & $\begin{array}{c}\text { HS } \\
\text { type }\end{array}$ & $\begin{array}{c}\text { FCD } \\
\text { type }\end{array}$ \\
\hline P1 & F & 26 & 25.6 & N & TLE & R & STG & Hippocampal sclerosis & 1 & N/A \\
P2 & F & 46 & 26.9 & N & TLE & L & STG & Hippocampal sclerosis & 2 & N/A \\
P3 & F & 50 & 25.6 & N & TLE & R & STG & Hippocampal sclerosis & 1 & N/A \\
P4 & M & 47 & 28.1 & N & TLE & R & STG & No abnormalities & N/A & N/A \\
P5 & F & 53 & 25.5 & N & TLE & L & STG & Meningioma and DNET & N/A & N/A \\
\hline
\end{tabular}

Table 4.1 Clinical and surgical data of included patients.

In this table, clinical and surgical data of the included patients are summarized.

BMI: body mass index, DNET: dysembryoplastic neuroepithelial tumor, F: female, FCD type: type of (focal) cortical dysplasia, HS type: type of hippocampal sclerosis according to ILAE classification, L: left, M: male, N: no, N/A: non-applicable, R: right, Smok: smoking, PA: results of histopathological examination, Side: side of surgery/measurement, STG: superior temporal gyrus, Y: yes.

\begin{tabular}{lcccccc}
\hline & $\begin{array}{c}\text { SBP } \pm \text { SEM } \\
(\mathrm{mmHg})\end{array}$ & $\begin{array}{c}\text { DBP }( \pm \text { SEM) } \\
(\mathrm{mmHg})\end{array}$ & $\begin{array}{c}\text { HR } \pm \text { SEM } \\
(\text { per minute })\end{array}$ & $\begin{array}{c}\text { Duration } \\
\text { (seconds) }\end{array}$ & Recordings & Sites \\
\hline M1 $(\mathrm{n}=5)$ & $95.4 \pm 3.8$ & $59.4 \pm 5.1$ & $62.8 \pm 3.9$ & $40.0 \pm 6.3$ & $16.0 \pm 1.1$ & $3287 \pm 27.0$ \\
$M 2(\mathrm{n}=4)$ & $111.0 \pm 4.6$ & $55.0 \pm 1.2$ & $58.5 \pm 3.6$ & $141 \pm 65.9$ & $27.5 \pm 2.3$ & $3263 \pm 44.8$ \\
M3 $(\mathrm{n}=5)$ & $112.2 \pm 7.7$ & $61.4 \pm 3.9$ & $65.2 \pm 5.4$ & $115.8 \pm 39.0$ & $27.0 \pm 5.0$ & $3213 \pm 70.7$ \\
\hline
\end{tabular}

Table 4.2 Clinical and technical measurements characteristics.

Hemodynamic parameters like SBP, DBP, and HR were recorded for each measurement. Besides a slightly lower SBP in $\mathrm{M} 1$ and $\mathrm{DBP}$ in $\mathrm{M} 2$, measurement hemodynamics did not vary. The duration of the cerebral measurements, $M 2$ and $M 3$, was substantially prolonged when compared to the sublingual measurement. At least 3000 vessel segments sites were required to complete a measurement. The number of recordings to obtain these sites was increased in the cerebral measurements.

DBP: diastolic blood pressure, HR: heart rate, M1: sublingual measurements, M2: cortical measurements, M3: hippocampal measurements, SBP: systolic blood pressure, SEM: standard error of mean. 


\subsection{Imaging procedure}

Directly following anesthesia induction and intubation with fixation of the endotracheal tube by adhesive plaster, sublingual measurements were carried out. Study subjects were in supine position and the camera was brought in orally along the endotracheal tube (Figure 4.1A). The central sublingual area was chosen for examination.

Next, incision, craniotomy, and durotomy were performed according to site of surgery and surgeon's preference. When the cortex was in view, the tip of the camera was wettened in Ringer's lactate and enclosed within the sterile slipcover. Blood clots were rinsed off the cortex using warm Ringer's Lactate. Finally, the camera was gently placed on the cortex (Figure 4.1B) and subtly fixed by the neurosurgeon to minimize pressure and movement artefacts. Contrary to sublingual measurements, this was a rather timeconsuming procedure in cortical measurements.

The anterior temporal lobe resection was performed subsequently. When the hippocampus was fully visualized, blood clots were rinsed off using warm Ringer's lactate. Next, the camera with sterile slipcover was gently placed on the lateral surface of the hippocampus and loosely fixed by the neurosurgeon (Figure 4.1C). Figure 4.2A and $4.2 \mathrm{~B}$, and video 1 show images of the microcirculation.

\subsection{Imaging procedure: issues and solutions}

In the first participant, we were not able to adequately visualize the cortical microcirculation. During this measurement, air bubbles were encountered, thereby obstructing acquisition of adequate focus to assess the microcirculation (Figure 4.2C). We assumed that the introduction of the sterile slipcover covering the camera enabled air bubbles to enter in between, even though the tip of the camera was wettened in Ringer's lactate before it was enclosed within the slipcover. Therefore, the conducting and pasting substance between the camera and slipcover was altered to sterile gel (Aquasonic sterile ultrasound transmission gel, Parker laboratories, Fairfield, USA). In the subsequently performed sterile hippocampal measurement, no air bubbles were noted. All sterile measurements thereafter were successfully completed using the gel instead of water. Nevertheless, we noted that it was sometimes difficult to properly visualize the cortical and hippocampal microcirculation and obtaining adequate focus appeared time consuming, independently of learning curve.

No (serious) adverse events were recorded during the measurement procedures or surgery. 


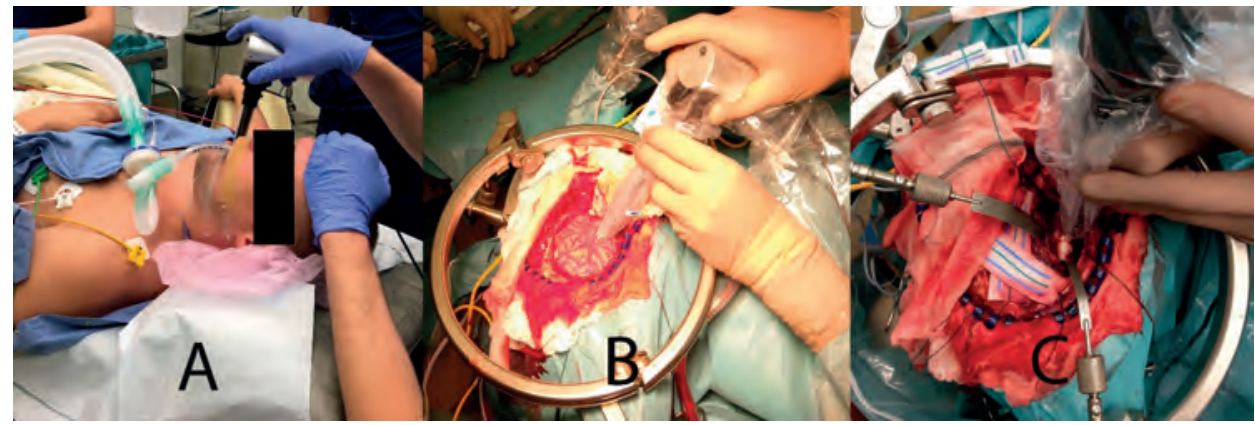

Figure 4.1 This figure exemplifies the position of the patient, measurement site, and handling of the Capiscope camera during the sublingual (A), cortical (B), and hippocampal (C) measurement. In Figure $4.1 \mathrm{~A}$, the tip of the camera is placed alongside the endotracheal tube. The round disk near the handgrip is used to obtain adequate focus. During the cortical (B) and hippocampal (C) measurement, the camera is enclosed within the sterile slipcover. One hand is placed around the camera tube to stabilize the camera and reduce movement artefacts. The other hand is used to obtain adequate focus. In Figure 4.1C, the white structure at the tip of the camera is the hippocampus. This structure is situated deeply within the cranium.

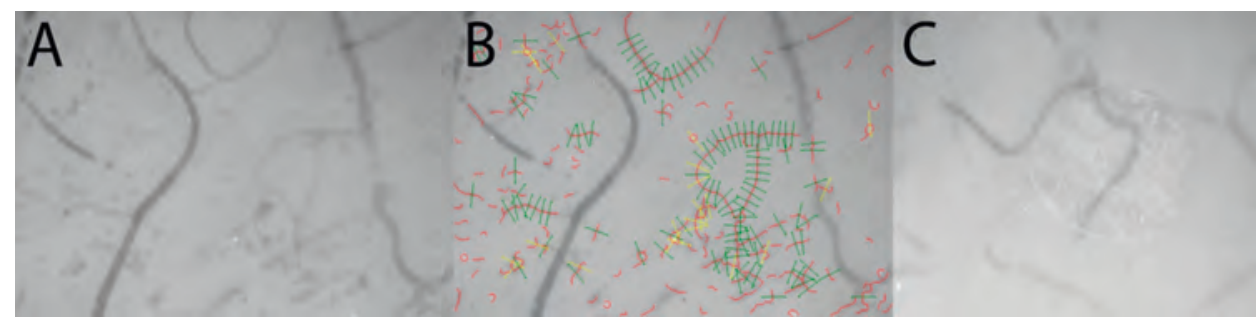

Figure 4.2 In Figure 4.2A, an image of the hippocampal microcirculation is shown. In Figure 4.2B, the similar image is depicted while analysis is performed using Glycocheck (C) software. In the latter, red lines reflect vessels recognized by the flowing erythrocytes within the vessels. Next, the red blood cell column (RBCC) width variation is estimated. The green lines oblique to the recognized vessels represent included RBCC width calculations whereas yellow lines oblique to the vessels were not successfully completed and therefore excluded. Figure $4.2 \mathrm{C}$ shows an air bubble obstructing the software to recognize vessels, despite adequate focus.

\subsection{Microvascular density}

The sublingual measurement was completed in all five participants. Mean sublingual vessel density was $274 \pm 47$ per $\mathrm{mm}^{2}$. Mean cortical vessel density $(\mathrm{n}=4)$ and hippocampal vessel density $(n=5)$ were substantially lower compared to the sublingual vessel density, $144 \pm 21$ per $\mathrm{mm}^{2}$ and $193 \pm 47$ per $\mathrm{mm}^{2}$, respectively. In Figure 4.3, microvascular density per RBCC diameter is presented. Mean sublingual vessel density is increased for all RBCC diameters when compared to the cortical and hippocampal vessel density. All three sites show a similar pattern, with a vessel increase of $7-10 \mu \mathrm{m}$ RBCC diameter classes. As can be seen in Figure 4.5 (graphs A-C-E-G-I), high intra- and 
interindividual variation was found between sublingual, cortical, and hippocampal vessel densities.

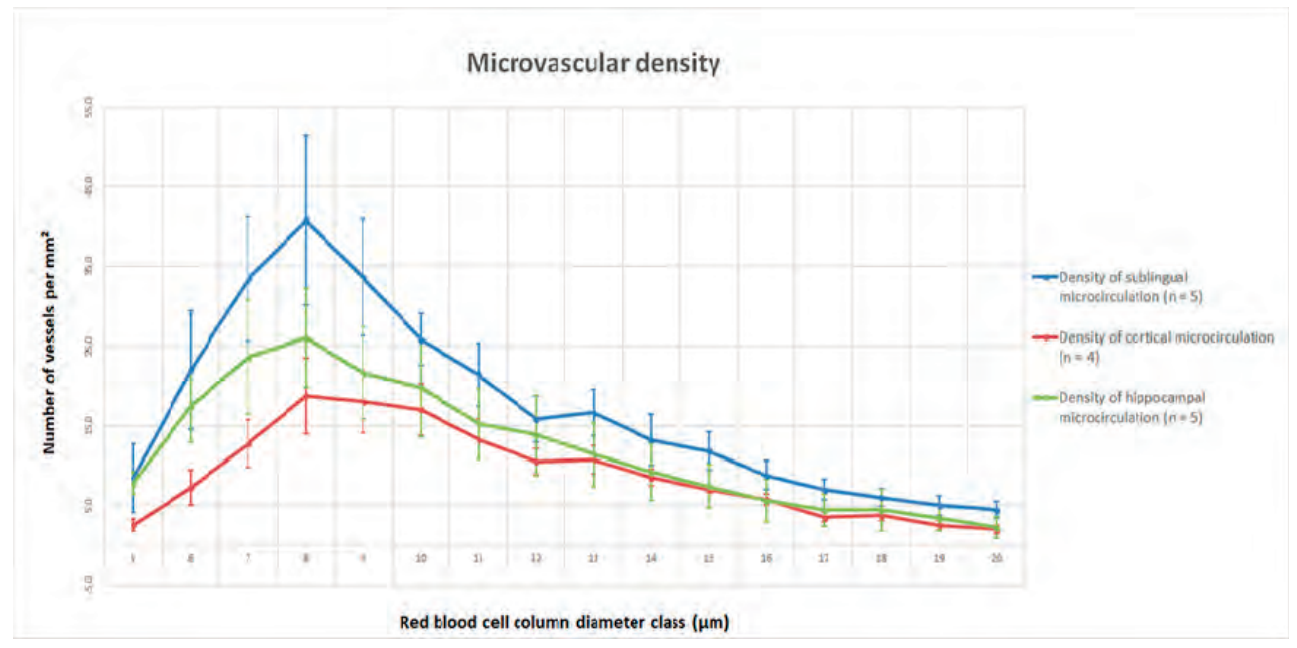

Figure 4.3 The mean number of microvessels is illustrated per red blood cell column (RBCC) diameter class. The sublingual (blue line) microvascular density is evidently higher in all RBCC diameter classes compared to the hippocampal (green line) and cortical (red line) microvascular density. Microvascular density of the latter is also substantially less than the hippocampal microvascular density in the lower RBCC diameter classes. Error bars shown reflect the standard error of mean.

\subsection{Perfused boundary region}

The mean sublingual PBR $(n=5)$ was $1.85 \pm 0.19 \mu \mathrm{m}$. The mean cortical PBR of the successfully assessed four patients was evidently increased, $2.28 \pm 0.18 \mu \mathrm{m}$. The mean hippocampal PBR ( $n=5$, TLE-patients) was $2.01 \pm 0.13 \mu \mathrm{m}$. Mean PBR values per RBCC diameter of all three sites are illustrated in Figure 4.4. This figure shows incremental PBR values in increasing vessel diameters. No correlation was found between the sublingual and cortical (Pearson correlation 0.425, $\mathrm{p}=0.58$ ) or hippocampal (Pearson correlation $-0.491, p=0.40$ ) PBR values (Figure 4.5, graphs B-D-F-H-J). 


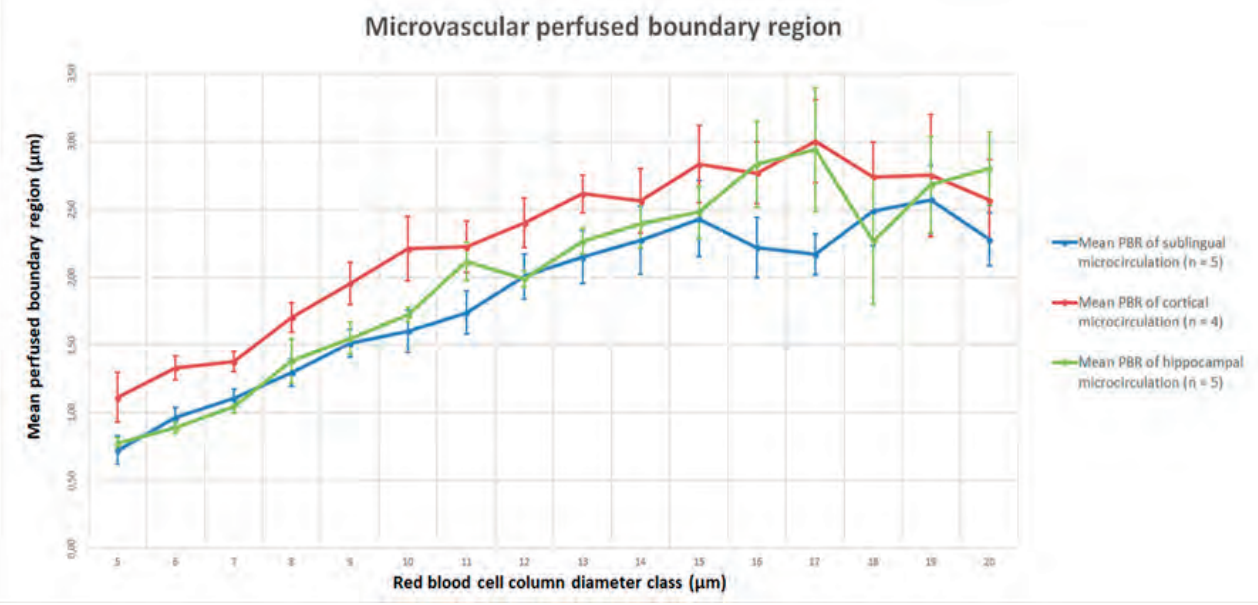

Figure 4.4 The perfused boundary region (PBR) is presented for all included red blood cell column (RBCC) diameter classes. Sublingual (blue line), cortical (red line), and hippocampal (green line) PBR values gradually increase with increasing RBCC diameter classes. PBR values of the cortical microcirculation are slightly increased between RBCC diameters of 5 and $15 \mu \mathrm{m}$ when compared to both, sublingual and hippocampal PBR values. Error bars shown reflect the standard error of mean.

\section{Discussion}

In this study, we have described a method to assess the human cerebral microcirculation and its glycocalyx during a craniotomy. Using SDF imaging, the cerebral microcirculation can be visualized easily, safely, and quickly. Subsequent analysis by Glycocheck (C) software, enabled us to analyze the cerebrovascular glycocalyx. The preliminary results on vessel density and PBR presented here, are provided to illustrate the feasibility of the measurements on cortical and hippocampal tissue.

\subsection{Evaluation of imaging procedure}

In general, visualization of the sublingual, cortical, and hippocampal microcirculation using SDF imaging is a safe technique that does not significantly prolong surgery or affect sterility. However, the sterile cerebral measurements were more difficult to obtain when compared to the sublingual. The entry of air bubbles between the camera and the sterile slipcover can be prevented by using a gel as pasting conductor. Nevertheless, the time to obtain adequate focus that was required to record data varied from 30 seconds up to 6 minutes in the cerebral measurements. We assume that this is mainly due to the introduction of the sterile slipcover. On the one hand, this slipcover alters fraction of the emitted and re-emitted light impeding adequate focus. 
On the other hand, the slipcover possibly disturbs image quality reducing the recognition of smaller vessels, as can be noted by lower vessel densities. This results in an increased number of recordings required to obtain at least 3000 vessel segments. Indeed, we noted vessel density of the cortical and hippocampal microcirculation was lower than sublingually and the number of recordings in the sterile cerebral measurements were markedly increase. Finally, physiological pulsations of the brain and cerebrospinal fluid that are not present sublingually may have contributed to the prolonged duration of cerebral measurements.

\subsection{Microvascular density}

Assessment of the human cerebral microcirculation has mainly been performed ex vivo by histologic evaluation ${ }^{23-26}$. Ex vivo assessment does not allow functional analysis of the microcirculation. Recently, SDF imaging and similar videomicroscopy techniques have been used to evaluate the human cerebral microcirculation ${ }^{28,29,32-35,41}$. In this study, we have confirmed these results by visualizing the functional sublingual, cortical, and hippocampal microcirculation and analyzing microvascular density. The sublingual vessel density was substantially higher than that in the hippocampus and even more so compared to that in the cortex. As was pointed out above, this might be due to the introduction of the sterile slipcover altering image acquisition properties.

We have tried to compare our results on the human cerebral vessel density to other reports using SDF imaging or its technical predecessor. However, most of these reports have mainly assessed parenchymal arterioles and venules with larger diameters (Mathura et al. 2001; Pennings et al. 2004, 2006, 2009). We found two reports on the vessel density of the cerebral microcirculation defined as RBCC diameters of 5-25 $\mu \mathrm{m}^{28,29}$. However, vessel density was expressed as perfused vessel density defined as the length (in millimeter) of perfused vessels of all diameters per $\mathrm{mm}^{2}$. Since we have analyzed the number of vessels per $\mathrm{mm}^{2}$, and differentiated this number per RBCC diameter, we cannot compare these results.

In our study, the distribution of RBCC diameters was comparable between all three sites with a predominant peak in the range of 7-10 $\mu \mathrm{m}$. As this is the first study characterizing cerebral microvascular densities at different cerebral parenchymal sites and comparing them to sublingual densities, we cannot relate these findings to other reports.

When sublingual vessel density would be comparable or correlated to cortical and/or hippocampal vessel density, the assessment of the sublingual microcirculation would suffice to predict cerebral microvascular densities. However, we found high intra- and interindividual variation of site-specific vessel densities reducing this predictive value. 

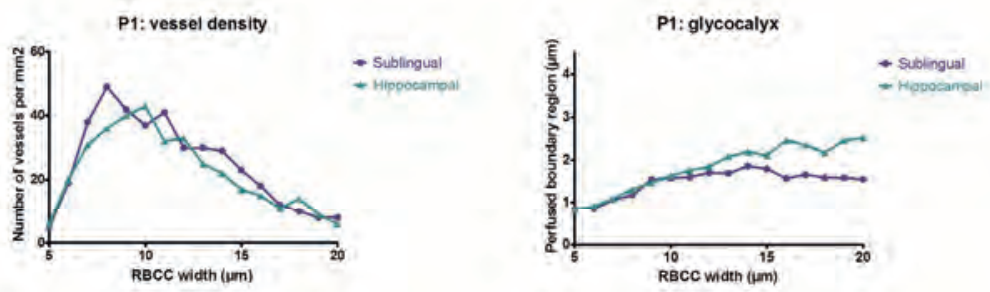

P2: vessel density
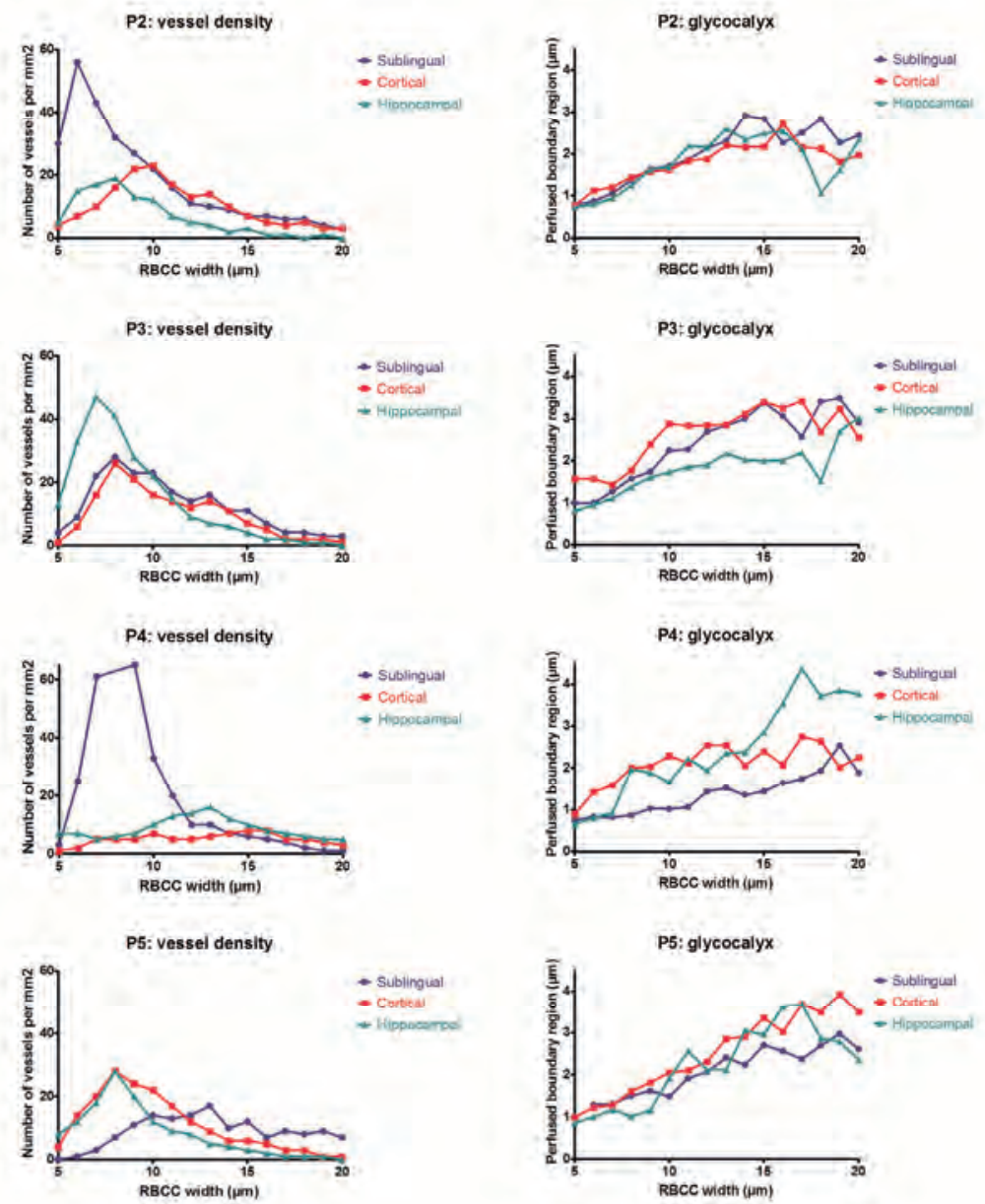

Figure 4.5 In the graphs on the left, vessel density per red blood cell column (RBCC) width of the sublingual (blue), cortical (red), and hippocampal (green) microcirculation are specified per patient. There is high intra- and interindividual variation between densities of these sites. The perfused boundary region (PBR) per RBCC width are also specified per patient. Incremental PBR values for increasing RBCC width can be noted, although no statistical correlation was found between the sublingual (blue), cortical (red), and hippocampal (green) PBR. 


\subsection{Perfused boundary region}

The glycocalyx is a matrix covering the luminal side of endothelial cells. It consists of proteoglycans and glycoproteins that provide a network in which other plasma- or endothelium-derived molecules are incorporated ${ }^{12}$. The endothelial side of the glycocalyx is more stable and firmly connected to the endothelium, whereas the apical side is more dynamic and permeable for plasma components like erythrocytes ${ }^{12,14}$. The PBR reflects this dynamic and permeable part of the glycocalyx. During inflammation or in cardiovascular disease, the glycocalyx is damaged and unstable which is characterized by a more dynamic and accessible PBR. Consequently, an increase of the RBCC width variation and PBR can be detected. Thus, PBR values illustrate health and stability of the glycocalyx.

Naturally, glycocalyx thickness and PBR values gradually increase with increasing vessel diameter, which is confirmed in our sublingual and cerebral observations. Furthermore, we found that PBR values of the sublingual and hippocampal microcirculation were rather well comparable, while cortical PBR values appeared increased between 5 and $15 \mu \mathrm{m}$ RBCC widths. This suggests a more permeable and unstable glycocalyx in the cortical microcirculation. However, the limited number of included patients reduces the effect of this interesting finding.

We found no correlation between sublingual, cortical, and hippocampal PBR values, probably due to the limited number of included patients. When sublingual PBR would be comparable or correlated to cortical and/or hippocampal PBR, the assessment of the sublingual microcirculation would suffice to estimate cerebral glycocalyx 'health'.

To the best of our knowledge, this is the first report on the in vivo assessment of the human cerebrovascular glycocalyx. Using two-photon laser scanning microscopy, the in vivo thickness of the cerebral endothelial glycocalyx has been evaluated in mice very recently ${ }^{42}$. To date, PBR has mainly been evaluated sublingually and has widely been accepted as a valid gauge of glycocalyx health ${ }^{9,12,16,43}$.

\subsection{Relevance of this technique}

In this paper, technical details of the assessment of the cerebral microcirculation and its glycocalyx are discussed as a potential tool to study the pathophysiology of underlying chronic drug-resistant epilepsy. Microcirculatory abnormalities in epilepsy patients include altered microvascular density, dysfunctional vessels, and inadequate neurovascular coupling $24,25,37,38$. Moreover, increased BBB permeability has been observed in epilepsy patients leading to astrocytic dysfunction and inflammation contributing to the reorganization of the local neuronal network, hyperexcitability, and dysfunctional neurovascular coupling in epilepsy ${ }^{37,39}$. Since the glycocalyx orchestrates vascular permeability, capillary perfusion, and plasma cell adhesion, it is imperative to further analyze the role of the glycocalyx in the pathophysiology of epilepsy. 
Furthermore, alterations in cerebral microvascular structures, neurovascular coupling, and BBB permeability have been implicated to play a role in various neurological disorders, including stroke, dementia, Alzheimer's disease, multiple sclerosis, and Parkinson's disease ${ }^{2,16,44-47}$. Epilepsy is one of the few chronic neurological disorders in which surgical treatment can be considered, thereby allowing in vivo assessment of the cerebral microcirculation and functionally important elements, like the glycocalyx. The current study may therefore contribute to unravel the pathophysiology of epilepsy and other neurological disorders, leading to novel therapeutic approaches. There is still need for this in epilepsy, because an estimated $30-40 \%$ of epilepsy patients are drugresistant ${ }^{48}$. The development of techniques to assess the cerebral microcirculation and its glycocalyx, as described in this report, is thus highly relevant.

\subsection{Limitations}

SDF imaging emits light at a wavelength absorbed by (de)-oxyhemoglobin, visualizing erythrocytes but not the vessel wall. Thus, vessel diameter and glycocalyx health and accessibility are measured indirectly, based on RBCC width variation and PBR, respectively.

Another technical limitation is the effect of the sterile slipcover on image quality. As was discussed earlier, an effect on inadequate recognition of vessels might influence results of both, vessel density and glycocalyx assessment.

Since the aim of this manuscript is technical, the included results are limited to a brief overview of only a few outcome parameters and should be interpreted with caution. Detailed results including a relation to clinical and histopathological parameters are presented when the study is completed.

\section{Conclusion}

This is the first report on the in vivo assessment of the human cerebrovascular glycocalyx. SDF imaging is a safe, quick, and straightforward technique to evaluate the cerebral microcirculation and its glycocalyx. Since the cerebral microcirculation and its glycocalyx play an eminent role in neurovascular coupling and accommodating cerebral homeostasis, this method may significantly advance research on the pathophysiology of various neurological disorders. 


\section{References}

1. Guo S, Lo EH. Dysfunctional Cell-Cell Signaling in the Neurovascular Unit as a Paradigm for Central Nervous System Disease. Stroke. 2009;40:S4-7.

2. Rosenberg GA. Neurological diseases in relation to the blood-brain barrier. J Cereb Blood Flow Metab. 2012;32:1139-51.

3. Abbott NJ, Patabendige AAK, Dolman DEM, Yusof SR, Begley DJ. Structure and function of the bloodbrain barrier. Neurobiol Dis. 2010;37:13-25.

4. Farrall AJ, Wardlaw JM. Blood-brain barrier: ageing and microvascular disease--systematic review and meta-analysis. Neurobiol Aging. 2009;30:337-52.

5. Brown WR, Thore CR. Review: Cerebral microvascular pathology in ageing and neurodegeneration. Neuropathol Appl Neurobiol. 2011;37:56-74.

6. Toth $\mathrm{P}$, Tarantini S, Csiszar A, Ungvari Z. Functional vascular contributions to cognitive impairment and dementia: mechanisms and consequences of cerebral autoregulatory dysfunction, endothelial impairment, and neurovascular uncoupling in aging. Am J Physiol Heart Circ Physiol. 2017;312(1): $\mathrm{H} 1-\mathrm{H} 20$

7. Iadecola C. Neurovascular regulation in the normal brain and in Alzheimer's disease. Nat Rev Neurosci. 2004;5:347-60.

8. Muoio V, Persson PB, Sendeski MM. The neurovascular unit - concept review. Acta Physiol. 2014;210:790-8.

9. Jacob M, Chappell D, Becker BF. Regulation of blood flow and volume exchange across the microcirculation. Crit Care. 2016;1-13.

10. Taccone FS, Su F, Pierrakos C, et al. Cerebral microcirculation is impaired during sepsis: an experimental study. Crit Care. 2010;14:R140.

11. Gould IG, Tsai P, Kleinfeld D, Linninger A. The capillary bed offers the largest hemodynamic resistance to the cortical blood supply. J Cereb Blood Flow Metab. 2017;37(1):52-68

12. Haeren RHL, van de Ven SEM, van Zandvoort MAMJ, et al. Assessment and imaging of the cerebrovascular glycocalyx. Curr Neurovasc Res. 2016;13(3):249-60

13. Ebong EE, Lopez-Quintero S V, Rizzo V, Spray DC, Tarbell JM. Shear-induced endothelial NOS activation and remodeling via heparan sulfate, glypican-1, and syndecan-1. Integr Biol. (Camb). 2014;6:338-47.

14. Reitsma S, Slaaf DW, Vink H, Van Zandvoort MAMJ, Oude Egbrink MGA. The endothelial glycocalyx: Composition, functions, and visualization. Pflugers Arch Eur J Physiol. 2007;454:345-59.

15. Rahbar E, Cardenas JC, Baimukanova G, et al. Endothelial glycocalyx shedding and vascular permeability in severely injured trauma patients. J Transl Med. 2015;13:1-7.

16. Martens RJH, Vink H, Van Oostenbrugge RJ, Staals J. Sublingual microvascular glycocalyx dimensions in lacunar stroke patients. Cerebrovasc Dis. 2013;35:451-4.

17. Vlahu CA, Lemkes BA, Struijk DG, Koopman MG, Krediet RT, Vink H. Damage of the endothelial glycocalyx in dialysis patients. J Am Soc Nephrol. 2012;23:1900-8.

18. Becker BF, Chappell D, Jacob M. Endothelial glycocalyx and coronary vascular permeability: The fringe benefit. Basic Res Cardiol. 2010;105:687-701.

19. Ostrowski SR, Berg RMG, Windel $\varnothing v$ NA, et al. Coagulopathy, catecholamines, and biomarkers of endothelial damage in experimental human endotoxemia and in patients with severe sepsis: A prospective study. J Crit Care. 2013;28:586-96.

20. Singh A, Fridén V, Dasgupta I, et al. High glucose causes dysfunction of the human glomerular endothelial glycocalyx. Am J Physiol Renal Physiol. 2011;300:F40-8.

21. de Mesy Bentley KL. An 11- $\mu$ m-thick glycocalyx?: it's all in the technique! Arterioscler. Thromb Vasc Biol. 2011;31:1712-3.

22. Hunsberger JG, Bennett AH, Selvanayagam E, Duman RS, Newton SS. Gene profiling the response to kainic acid induced seizures. Mol Brain Res. 2005;141:95-112.

23. Pitkänen A, Lukasiuk K. Molecular and cellular basis of epileptogenesis in symptomatic epilepsy. Epilepsy Behav. Elsevier Inc.; 2009;14:16-25.

24. Rigau V, Morin M, Rousset M-C, et al. Angiogenesis is associated with blood-brain barrier permeability in temporal lobe epilepsy. Brain. 2007;130:1942-56. 
25. Kastanauskaite A, Alonso-Nanclares L, Blazquez-Llorca L, Pastor J, Sola RG, DeFelipe J. Alterations of the microvascular network in sclerotic hippocampi from patients with epilepsy. J Neuropathol Exp Neurol. 2009;68:939-50.

26. Morin-Brureau M, Lebrun A, Rousset $M-C$, et al. Epileptiform activity induces vascular remodeling and zonula occludens 1 downregulation in organotypic hippocampal cultures: role of VEGF signaling pathways. J Neurosci. 2011;31:10677-88.

27. Dane MJC, van den Berg BM, Lee DH, et al. A microscopic view on the renal endothelial glycocalyx. Am J Physiol Ren Physiol. 2015;308(9):F956-66.

28. Pérez-Bárcena J, Goedhart P, Ibáñez J, et al. Direct observation of human microcirculation during decompressive craniectomy after stroke. Crit Care Med. 2011;39:1126-9.

29. Pérez-Bárcena J, Romay E, Llompart-Pou JA, et al. Direct observation during surgery shows preservation of cerebral microcirculation in patients with traumatic brain injury. J Neurol Sci. 2015;353:38-43.

30. Wan Z, Ristagno G, Sun S, Li Y, Weil MH, Tang W. Preserved cerebral microcirculation during cardiogenic shock. Crit Care Med. 2009;37:2333-7.

31. Pennings F a, Ince C, Bouma GJ. Continuous real-time visualization of the human cerebral microcirculation during AVM surgery using orhogonal polarization spectral imaging. Neurosurgery. 2006;58:167-71.

32. Pennings $\mathrm{F}$ a, Bouma GJ, Ince C. Direct observation of the human cerebral microcirculation during aneurysm surgery reveals increased arteriolar contractility. Stroke. 2004;35:1284--8.

33. Pennings FA, Albrecht KW, Muizelaar JP, Schuurman PR, Bouma GJ. Abnormal responses of the human cerebral microcirculation to papaverin during aneurysm surgery. Stroke. 2009;40:317-20.

34. Uhl E, Lehmberg J, Steiger H-J, Messmer K. Intraoperative detection of early microvasospasm in patients with subarachnoid hemorrhage by using orthogonal polarization spectral imaging. Neurosurgery. 2003;52:1307-17.

35. Mathura KR, Bouma GJ, Ince C, Patterson J, Bollinger A, Ince C. Abnormal microcirculation in brain tumours during surgery. Lancet. Springer-Verlag, Berlin, Heidelberg; 2001;358:1698-9.

36. Alonso-Nanclares L, DeFelipe J. Alterations of the microvascular network in the sclerotic hippocampus of patients with temporal lobe epilepsy. Epilepsy Behav. Elsevier Inc.; 2014;38:48-52.

37. Heinemann $U$, Kaufer D, Friedman A. Blood-brain barrier dysfunction, TGF $\beta$ signaling, and astrocyte dysfunction in epilepsy. Glia. 2012;60:1251-7.

38. Parfenova H, Carratu P, Tcheranova D, Fedinec A, Pourcyrous M, Leffler CW. Epileptic seizures cause extended postictal cerebral vascular dysfunction that is prevented by HO-1 overexpression. AJP Hear Circ Physiol. 2005;288:H2843-50.

39. van Vliet EA, Aronica E, Gorter JA. Role of blood-brain barrier in temporal lobe epilepsy and pharmacoresistance. Neuroscience. 2014;277:455-73.

40. Haeren RHL, Vink $\mathrm{H}$, Staals J, et al. Protocol for intraoperative assessment of the human cerebrovascular glycocalyx. BMJ Open. 2017;7:e013954.

41. Pennings FA, Ince C, Bouma GJ. The arteriovenous malformation study group: Current concepts: arteriovenous malformations of the brain in adults. NEJM. Oxford University Press; 2006;340:1812-8.

42. Yoon J-H, Lee E-S, Jeong Y. In vivo Imaging of the Cerebral Endothelial Glycocalyx in Mice. J. Vasc. Res. 2017;54:59-67.

43. Dane MJC, Khairoun MR, Hyun Lee $D$, et al. Association of kidney function with changes in the endothelial surface layer. Clin J Am Soc Nephrol. 2014;9:698-704.

44. Ortiz GG, Pacheco-Moisés FP, Macías-Islas MÁ, et al. Role of the blood-brain barrier in multiple sclerosis. Arch Med Res. 2014;45:687-97.

45. Zhang JH, Badaut J, Tang J, Obenaus A, Hartman R, Pearce WJ. The vascular neural network--a new paradigm in stroke pathophysiology. Nat Rev Neurol. 2012;8:711-6.

46. Bell RD, Zlokovic B V. Neurovascular mechanisms and blood-brain barrier disorder in Alzheimer's disease. Acta Neuropathol. 2009;118:103-13.

47. Erdő F, Denes L, de Lange E. Age-associated physiological and pathological changes at the blood-brain barrier: A review. J Cereb Blood Flow Metab. 2017;37:4-24.

48. Picot $M C$, Baldy-Moulinier $M$, Daurès JP, Dujols $P$, Crespel $A$. The prevalence of epilepsy and pharmacoresistant epilepsy in adults: A population-based study in a Western European country. Epilepsia. 2008;49:1230-8. 


\section{Chapter 5}

Cerebral artery vasoconstriction requires
neurogenic and adrenergic interaction and
is endothelin-1 dependent

R.H.L. Haeren, S.A. Hartmans, J. De Mey, G. Hoogland, J. Dings, O.E.M.G. Schijns, S.M.J. van Kuijk, K. Rijkers, P. Schiffers, J.J. van Overbeeke Adapted from the publication in Current Neurovascular Research, 2017 


\section{Structured abstract}

\section{Background}

The regulation of cerebral arterial vasomotor tone involves several mechanisms. The role of sympathetic nerves and the adrenergic neurotransmitter, noradrenaline (NA), has been subject of debate for decades. Moreover, the specific role of endothelin-1 (ET-1) in cerebral arterial vasoconstriction has not been elucidated to date.

\section{Objective}

In this study, we evaluated the contribution of NA and ET-1 to cerebral artery vasoconstriction.

\section{Method}

Arterial responses of rat middle cerebral arteries, and human pial cerebral arteries to cumulative concentrations of NA and ET-1, and to electrical field stimulation (EFS), were evaluated. To assess the role of NA and ET-1 when EFS was applied, experiments were performed in the presence of adrenergic, neurogenic, and $\mathrm{ET}_{\mathrm{A}}$-receptor modulators.

\section{Results}

We found that vasoconstriction of cerebral arteries following EFS requires the application of exogenous NA, whereas neither EFS nor NA alone induced vasoconstriction. The observed vasoconstriction was abolished by $\alpha$-adrenoreceptor antagonist, catecholamine-release inhibitor, blockade of the perivascular neurons, and by the $\mathrm{ET}_{\mathrm{A}}$-receptor antagonist (BQ123).

\section{Conclusion}

Based on our results, cerebral artery vasoconstriction requires simultaneous neurogenic and adrenergic activation and is ET-1 dependent. We hypothesize that NA modulates the release of $\mathrm{ET}-1$. Upon release, $\mathrm{ET}-1$ binds to the $\mathrm{ET}_{\mathrm{A}}$-receptor on smooth muscle cells inducing cerebral artery vasoconstriction. 


\section{Introduction}

The regulation of cerebral blood flow involves several mechanisms including endocrine, metabolic, neurogenic, and endothelial ones ${ }^{1-7}$. Pivotal in this regulation is the vasomotor tone, which is the amount of tension in the smooth muscle cells within the vessel wall. In this paper, we focus on neurogenic and endothelial control of cerebral artery vasomotor tone.

\subsection{Neurogenic control of vasomotor tone}

Neurogenic vasomotor control is exerted by perivascular nerve fibers embedded within the adventitia of cerebral arteries ${ }^{1,3,8}$. Neurotransmitters released by these perivascular nerves act on arterial smooth muscle cells inducing vasoconstriction or vasodilation. Classically, sympathetic adrenergic perivascular innervation is held responsible for most of the vasoconstrictor tone ${ }^{9-12}$. However, there has always been debate on the precise vasomotor influence of sympathetic nerves and noradrenaline (NA) in cerebral arteries: some have reported strong contractile responses to stimulation of perivascular sympathetic nerves, while others have found no response at all. ${ }^{8,13-19}$. This has mainly been attributed to highly variable sympathetic nerve and receptor densities, and to the release of other neurotransmitters ${ }^{15,17,19-21}$.

\subsection{Endothelial control of vasomotor tone}

Endothelial cells also release important vasoactive substances including nitric oxide (NO), endothelial-derived hyperpolarizing factor and endothelin-1 (ET-1) ${ }^{4,22-25}$. ET-1 is probably the most potent endothelial vasoconstrictor but is also expressed by perivascular neurons ${ }^{26-28}$. ET-1 mainly exerts its vasoconstrictor effects by binding to $\mathrm{ET}_{\mathrm{A}}$-receptors on smooth muscle cells activating phospholipase $\mathrm{C}$ which stimulates intracellular calcium release and calcium influx ${ }^{29}$. In contrast, $\mathrm{ET}_{\mathrm{B}}$-receptors are predominantly expressed by endothelial cells. They play a role in scavenging circulating ET-1, the production of NO resulting in vasodilatation, and in extravasation of monocytes $^{30,31}$.

Pagán et al. recently described that vasoconstriction induced by electrical field stimulation (EFS) was reduced following the administration of an ET-1 antagonist in internal mammary arteries ${ }^{12}$. Similar results have previously been reported on the contractile responses of cerebral arteries ${ }^{29,32-35}$. Based on these results, ET-1 is suggested to play a role in vasoconstriction of cerebral arteries.

\subsection{Aim of this study}

In this study, we hypothesized that vasoconstrictor effects of NA and EFS in the cerebrovascular bed are mediated by ET- $1^{36}$. To address this hypothesis, we analyzed 
the regulation of cerebral vasomotor tone by studying the response of cerebral arteries to EFS in the absence and presence of several adrenergic and neurogenic modulators, and ET-1 agonists and antagonists.

These studies were performed in human cerebral arterial segments that were part of remnant biopsies, obtained during resective surgery for epilepsy. Epilepsy is a chronic neurological disorder characterized by recurrent unprovoked seizures ${ }^{37}$. The increased neuronal activity characterizing seizures is accompanied by a high metabolic demand, which can only be maintained by adaptation of local cerebral blood flow. This cerebral blood flow adaptation is regulated by changing vasomotor tone and requires adequate functioning of autoregulation and neurovascular coupling. In epilepsy, a disturbed neurovascular coupling has been described, yet the underlying mechanisms of this dysfunctional coupling have not been studied to date ${ }^{38-40}$. In order to assess whether vasomotor control alterations are apparent in cerebral arteries of epilepsy patients, we compared the results in cerebral arteries from epilepsy patients to those in control rats.

\section{Materials and methods}

For this study, both human and animal brain tissue was used. Experimental protocols of human tissue-handling were in accordance with Good Clinical Practice guidelines. Since the collected human arterial segments were part of remnant, otherwise discarded biopsies, this study did not require medical ethical approval according to the Dutch Law: Wet Medisch-wetenschappelijk Onderzoek. Nevertheless, a signed informed consent was obtained preoperatively in all cases. All animal experimental protocols were in accordance with institutional guidelines, were approved by the local ethical committee for experimental animal welfare of Maastricht University, and are reported according ARRIVE guidelines.

\subsection{Tissue samples}

Neocortical samples of adult patients undergoing resective brain surgery for drugresistant epilepsy were collected during surgery. Samples were immediately stored in room temperature HEPES-buffered physiologic salt solution (HEPES-PSS). Subsequently, human pial arteries (HPAs) were isolated by microdissection. All HPA experiments were performed within 2-3 hours after resection.

Male Wistar-Kyoto rats (Charles-River, Maastricht, the Netherlands) were housed in individual cages, and maintained on a 12-hour light/dark cycle, having access to standard laboratory rat chow and water ad libitum. At sixteen weeks of age, they were euthanized using $\mathrm{CO}_{2}$ inhalation. Within 1-2 hours after euthanizing, first order segments of the rat middle cerebral arteries (MCAs) were isolated. Since rat mesenteric arteries (RMAs) have been found to respond well to EFS, first order RMAs were included as a reference for the experiments with $\mathrm{EFS}^{10}$. 


\subsection{Electrical field stimulation}

Arterial segments with an average length of 2-3 $\mathrm{mm}$ were mounted between two glass micropipettes (100-125 $\mu \mathrm{m}$ ) of an arteriograph organ chamber (Living Systems Instrumentation, Burlington, VT, USA). Residual luminal blood was flushed using HEPESPSS. The organ chamber was filled with HEPES-PSS and kept at a constant temperature of $37{ }^{\circ} \mathrm{C}$. Using a video-dimension analyzer the arterial diameter was continuously monitored while transmural pressure was maintained at $70 \mathrm{mmHg}$. Arterial segments were rested for 30 minutes to gain myogenic tone. Subsequently, arterial viability and endothelial functional integrity was verified by assessing the acute dilatation induced by acetylcholine (ACh, $10 \mu \mathrm{M}$ ) following vasoconstriction induced by high potassium PSS (K-PSS, $60 \mathrm{mM})^{10}$.

EFS was applied by two platinum electrodes, placed along the longitudinal axes of the arterial segments at an inter-electrode distance of $7 \mathrm{~mm}$, and connected to a microstimulator (Danish Myotechnology, Aarhus, Denmark) ${ }^{10}$. The EFS protocol consisted of increasing frequencies, ranging from 0.5 to $32 \mathrm{~Hz}$, during 30 seconds for each frequency (pulse duration $2 \mathrm{~ms}$, pulse intensity $85 \mathrm{~mA})^{10}$. EFS was first applied to RMAs to assess our EFS set-up and parameters.

\subsection{Pharmacological strategy}

First, cumulative concentration-response curves (CCRC) of MCAs and HPAs to noradrenaline (NA, $\alpha_{1} / \alpha_{2}$-adrenoceptor agonist), serotonin (5-HT, a 5-HT1 and 5-HT2 receptor agonist), and endothelin-1 (ET-1, an $\mathrm{ET}_{\mathrm{A}}$ and $\mathrm{ET}_{\mathrm{B}}$ receptor agonist) were recorded with cumulative concentrations from $0.01 \mu \mathrm{M}$ up to $20 \mu \mathrm{M}$ for NA and 5-HT, and $0.01 \mathrm{nM}$ up to $0.65 \mathrm{nM}$ for ET-1.

Next, EFS was performed in absence of any pharmacological substances, further referred to as the initial response. Because of lack of response to EFS in MCAs and HPAs, all subsequent EFS experiments were performed in presence of exogenous NA at a concentration of $10 \mu \mathrm{M}$.

Thereafter, EFS was applied in presence of guanethidine (Gua, catecholamine release inhibitor), phentolamine (Phen, $\alpha_{1} / \alpha_{2}$ adrenoceptor antagonist), tetrodotoxin (TTX, inhibitor of neuronal sodium $\left(\mathrm{Na}^{+}\right)$channels), or $\mathrm{BQ123}$ (selective $\mathrm{ET}_{\mathrm{A}}$ receptor antagonist).

The following drugs were used: NA (CCRC or $10 \mu \mathrm{M})$, 5-HT (CCRC), Gua (5 $\mu \mathrm{M})$, Phen $(1 \mu \mathrm{M}), \mathrm{BQ} 123(1 \mu \mathrm{M})$, and $\mathrm{ACh}(10 \mu \mathrm{M})$ (all from Sigma-Aldrich, Zwijndrecht, the Netherlands). In addition, we have used ET-1 (CCRC) (Bachem, Weil am Rein, Germany) and TTX (1 $\mu \mathrm{M})$ (ToCris, Bristol, United Kingdom).

All compounds were applied abluminal, i.e. into the organ chamber in which the arterial segments were mounted. 


\subsection{Solutions}

HEPES-PSS was continuously maintained at a temperature of $37^{\circ} \mathrm{C}$, and contained in $\mathrm{mM}: \mathrm{KCl}$ 4.7, $\mathrm{NaCl} 144, \mathrm{CaCl}_{2} 2.5, \mathrm{KH}_{2} \mathrm{PO}_{4}$ 1.2, $\mathrm{MgSO}_{4}$ 1.2, HEPES 14.9, glucose 5.5, at $\mathrm{pH}$ 7.4. K-PSS was also maintained at $37^{\circ} \mathrm{C}$, and contained identical compound concentrations as HEPES-PSS, except for $\mathrm{KCl} 60 \mathrm{mM}$, and $\mathrm{NaCl} 86 \mathrm{mM}$, at $\mathrm{pH} 7.4$.

\subsection{Immunohistochemistry}

Parts of pressurized HPAs were fixed for 48 hours in buffered $4 \%$ formaldehyde solution at room temperature and embedded in paraffin. The presence of ET- 1 was determined by immunohistochemically staining. Cross-sections of 5 micrometer thick were incubated for six hours at $4^{\circ} \mathrm{C}$ with primary antibody against ET-1 (1:2000, 803-001R100, Alexis corporation, Lausen, Switzerland), followed by mouse immunoglobulin biotin (1:400, E0464, Dako A/S, Denmark) for 30 minutes at room temperature. Colorimetric detection was completed with 3,3'-diaminobenzidine (Boehringer Mannheim $\mathrm{GmbH}$, Mannheim, Germany), and sections were counterstained with Mayer's haematoxylin (Sigma-Aldrich).

\subsection{Data and statistical analysis}

Baseline characteristics of patients were described using age, sex, body mass index (BMI), use of anti-epileptic drug (AED use), and the application in pharmacological experiments. Diameter responses to cumulative concentrations of agonists are shown as semi-logarithmic concentration-response curves. The agonist concentration required for half-maximal response $\left(E C_{50}\right.$, expressed as $\mathrm{pD}_{2}=-\log E C_{50}$ ) and the maximal response $\left(E_{\max }\right)$ are shown as mean \pm standard error of mean (SEM).

Responses to EFS are presented as the maximal effect $\left(E_{\max }\right)$ at $32 \mathrm{~Hz}$ and expressed as the mean percentage and SEM of lumen diameter change relative to the initial lumen diameter in the absence of stimulation $(0 \mathrm{~Hz})$. The $E_{\max }$ are shown in bar graphs. We used the paired T-test to evaluate differences measured on the same arterial lumen following incubation of the pharmacological substances and with increasing EFS frequencies. ANCOVA analyses were performed to evaluate differences between rat MCAs and HPAs responses in the CCRC set-up and following incubation of the pharmacological substance after EFS and NA application. Data analysis was performed using GraphPad Prism 5 (GraphPad Software, San Diego, CA, USA). A p value $\leq 0.05$ was considered to indicate statistical significance. 


\section{Results}

\subsection{General observations}

A total of 5 RMAs (median diameter $=377 \mu \mathrm{m}$ ) of 5 rats, 22 MCA segments (median diameter $=237 \mu \mathrm{m}$ ) of 16 rats, and 21 HPA segments (median diameter $=407 \mu \mathrm{m}$ ) of 14 patients (Table 5.1), were included. Included arterial segments showed vasoconstrictor responses $>50 \%$ to K-PSS, and dilatation in response to subsequent ACh (10 $\mu \mathrm{M})$ application.

\begin{tabular}{lcccccc}
\hline Patient & Age & Sex & BMI & Smoking & AED use & Applied drug experiments \\
\hline 1 & 41 & F & 20.7 & N & CBZ, LVT & NA, Gua \\
2 & 65 & F & 27.7 & Y & CBZ & NA, Gua \\
3 & 70 & M & 28.1 & N & CLB, PHE, VAL & NA, Gua \\
4 & 29 & M & 26.3 & Y & CBZ, LAC, LVT & NA, Gua \\
5 & 35 & M & 30.0 & N & CLB, OXC & NA, Gua \\
6 & 43 & F & 27.2 & Y & CBZ, LAC & NA, Gua \\
7 & 43 & F & 19.6 & N & CBZ & NA, Gua \\
8 & 62 & M & 30.7 & N & CLB, LAM, LVT, ZON & NA, TTX, BQ123 \\
9 & 65 & M & 24.6 & N & CLB, OXC & NA, TTX, BQ123 \\
10 & 57 & M & 22.6 & N & CLB, CBZ, LVT & NA, TTX, BQ123 \\
11 & 35 & M & 30.3 & N & CBZ & NA, TTX, BQ123 \\
12 & 19 & M & 22.6 & N & CLB, LVT, OXC, VAL & NA, TTX, BQ123 \\
13 & 18 & F & 26.9 & N & LVT & NA, TTX, BQ123 \\
14 & 29 & F & 29.8 & N & LAC & NA, TTX, BQ123 \\
\hline
\end{tabular}

Table 5.1 Demographic characteristics of epilepsy patients that were included in the human pial artery (HPA) experiments. Also, the pharmacological agents applied for each patient are depicted.

BMI: Body mass index; AED: Anti-epileptic drug; EFS: Electrical field stimulation; M: Male; F: Female; Y: Yes; N: No; CBZ: Carbamazepine; CLB: Clobazam; LAC: Lacosamide; LAM: Lamotrigine; LVT: Levetiracetam; OXC: Oxcarbazepine; PHE: Phenytoin; VAL: Valproic acid; ZON: Zonisamide, NA: Noradrenaline; Gua: Guanethidine; TTX: Tetrodotoxine; BQ123: ETA-receptor antagonist.

First, we evaluated our EFS set-up by applying EFS to RMAs $(n=5)$ as has been reported earlier by our group ${ }^{10,29}$. A maximal vasoconstriction response of $28.2 \pm 6.7 \%$ was noted following EFS which was partially counteracted by the addition of Gua to $86.5 \pm 6.2 \%$ $(p=0.18)$ of the initial diameter (Figure 5.1). 


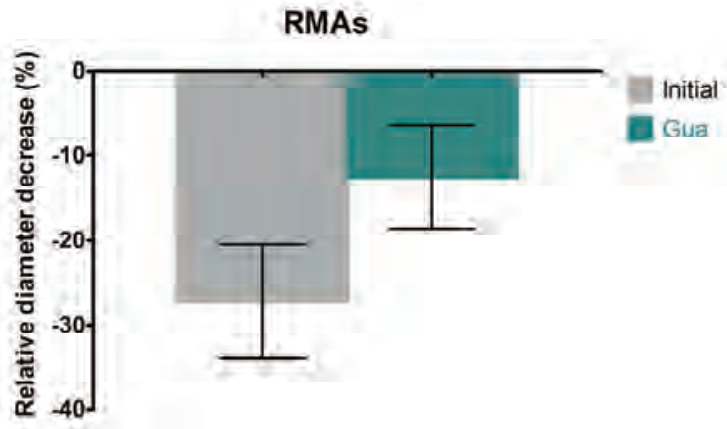

Figure 5.1 Vasomotor response of rat mesenteric arteries (RMA; $\mathrm{n}=5$ ) to $32 \mathrm{~Hz}$ electrical field stimulation. The initial response, i.e. in the absence of any pharmacological substance, resulted in a decrease of the initial diameter (grey bar). The subsequent addition of Guanethidine (Gua) partially reversed this EFS-induced vasoconstriction. Data are expressed as mean \pm SEM percentage change of the initial diameter.

\subsection{Cumulative concentration-response curves to NA, 5-HT and ET-1}

Noradrenaline induced minor concentration dependent vasoconstriction in MCAs $\left(E_{\max }=12.9 \pm 2.1 \% ; \mathrm{pD}_{2}=5.09 ; \mathrm{n}=12\right)$ and $\mathrm{HPAs}\left(\mathrm{E}_{\max }=11.5 \pm 4.8 \% ; \mathrm{pD}_{2}=6.24 ; \mathrm{n}=8\right)$. Cumulative concentrations of $5-\mathrm{HT}$ induced strong vasoconstriction in $\mathrm{MCAs}\left(\mathrm{E}_{\max }=\right.$ $\left.60.9 \pm 1.7 \% ; \mathrm{pD}_{2}=6.34 ; \mathrm{n}=10\right)$ and $\mathrm{HPAs}\left(\mathrm{E}_{\max }=20.7 \pm 7.2 \% ; \mathrm{pD}_{2}=7.64 ; \mathrm{n}=5\right) . \mathrm{ET}-1$ induced the largest response in $\mathrm{MCAs}\left(\mathrm{pD}_{2}=6.99 ; n=7\right)$ and $\mathrm{HPAs}\left(\mathrm{pD}_{2}=7.40 ; n=4\right)$, with a respective diameter decrease of $70.8 \pm 1.6 \%$ and $40.6 \pm 9.5 \%$ (Figure 5.2 ).
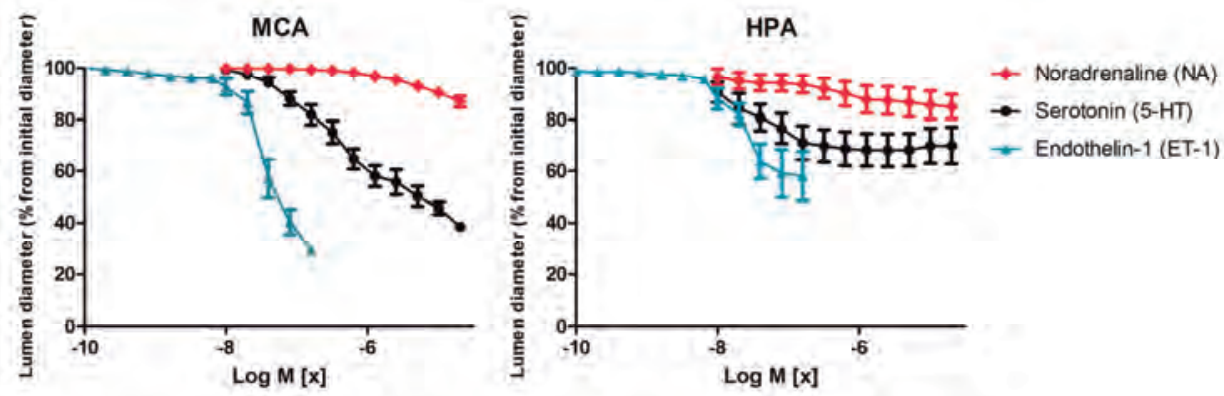

Figure 5.2 Cumulative concentration response curves (CCRC) of vasoconstrictive responses to noradrenaline (NA), serotonin (5-HT) and endothelin-1 (ET-1) in rat middle cerebral artery (MCA, left image) and human pial arteries (HPA, right image). In both, MCA ( $n=7$ to 12 ) and HPA ( $n=4$ to 8$), E T-1$ and 5-HT induced a strong decrease in diameter, whereas NA only caused a minor vasoconstriction. Data are expressed as mean \pm SEM percentage of initial diameter. 


\subsection{EFS and vasoactive modulators in rat middle cerebral arteries (Figure 5.3)}

In rat MCAs, application of EFS in the absence of any pharmacological substance, i.e. the initial response, induced a small change in arterial diameter; $E_{\max } 7.9 \pm 3.2 \%$ $(p=0.043 ; n=7)$. The subsequent application of EFS in the presence of exogenous NA, resulted in a far more reduced mean MCA lumen diameter when compared to the initial response; $E_{\max } 21.1 \pm 6.9 \%(p=0.022 ; n=7)$. The role of exogenous NA was further assessed by the addition of Gua, which did not alter vasoconstrictor responses $\left(E_{\max }\right)$, not affecting lumen diameter significantly $(1.2 \pm 7.8 \%(p=0.901 ; n=7))$. The addition of Phen completely abolished vasoconstriction, increasing lumen diameter to $4.7 \pm 3.4 \%$ $(p=0.021 ; n=6)$ larger than the initial diameter.

Next, the effect of TTX on the synergistic vasoconstrictive effect of EFS and exogenous NA, was assessed. An increase of $12.3 \pm 5.2 \%(p=0.270 ; n=6)$ in mean lumen diameter was noted in rat MCAs. The role of ET-1 in the vasoconstrictor response was assessed by the application of $\mathrm{BQ123}$, a selective $\mathrm{ET}_{\mathrm{A}}$ receptor antagonist, after vasoconstriction was evoked by EFS and exogenous NA. A vasoconstriction of $26.3 \pm 0.3 \%$ ( $p=0.019$; $\mathrm{n}=5$ ), was fully counteracted, increasing mean lumen diameter to $100.0 \%$ of the initial diameter.

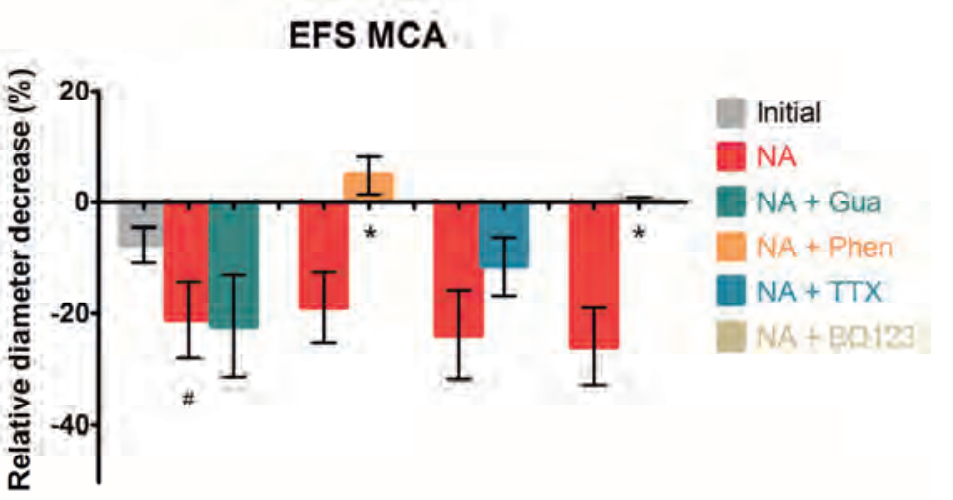

Figure 5.3 Maximal vasomotor responses of rat middle cerebral arteries (MCA) to electrical field stimulation (EFS) at $32 \mathrm{~Hz}$. The initial response in absence of any pharmacological agent (grey bar) only showed a small vasoconstriction $(n=7)$. The application of exogenous noradrenaline (NA) markedly increased the vasoconstrictor effect of EFS ( $n=7)$. Guanethidine (Gua) did not modify this effect of NA $(n=7)$ in MCAs. Subsequently, tetrodotoxin (TTX) reduced the vasoconstrictive effect of EFS in combination with exogenous NA $(n=6)$, and both phentolamine (Phen, $n=6)$ ) and BQ123 ( $n=5)$ fully abolished this effect. Data are expressed as mean \pm SEM percentage change of the initial diameter.

\#; $\mathrm{p} \leq 0,05$ when compared to the initial response. *; $\mathrm{p} \leq 0,05$ when compared to EFS in combination with exogenous NA 


\subsection{EFS and vasoactive modulators in human pial arteries}

Results of EFS and vasoactive modulators in HPAs are presented in Figure 5.4. The application of EFS alone did not cause a vasoconstrictor response in HPAs; $E_{\max } 2.0 \pm 2.0 \%$ $(n=7)$. The combination of exogenous NA and EFS resulted in a significant decrease of mean arterial lumen diameter of $50.9 \pm 3.7 \%(p=0.016 ; n=7)$.

This vasoconstrictor response was partially counteracted by the addition of Gua, increasing mean lumen diameter $\left(E_{\max }\right)$ with $24.3 \pm 6.3 \%$ to $75.2 \%(p=0.047 ; n=7)$ of the initial diameter. Due to the limited number of HPA segments, the subsequent application of Phen to exogenous NA and EFS was not included.

The synergistic effect of EFS and exogenous NA was neutralized by the application of TTX, increasing mean lumen diameter with $37.6 \pm 2.8 \%$ to $90.0 \%(p=0.003 ; n=7)$ of the initial diameter. The application of $\mathrm{BQ} 123$ also resulted in a nearly complete neutralization of vasoconstriction to $96.5 \pm 1.5 \%(p=0.005 ; n=7)$ of the initial diameter.

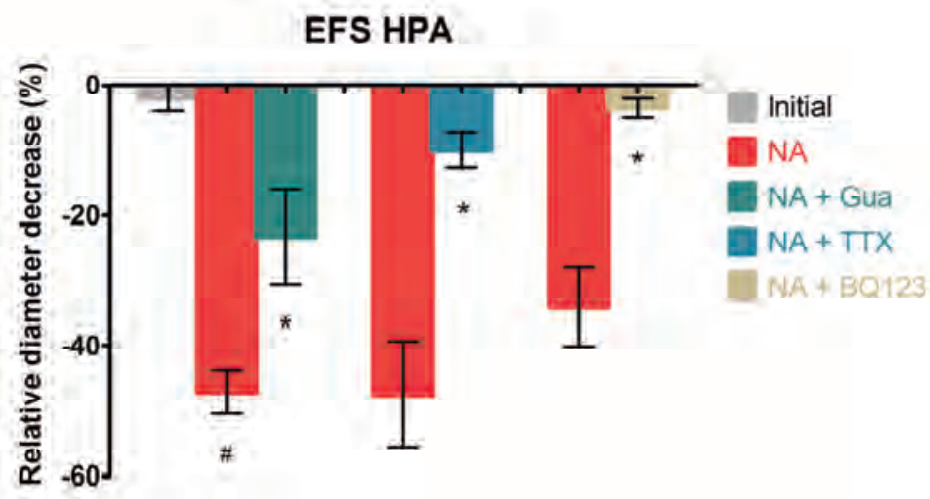

Figure 5.4 Maximal vasomotor responses of human pial arteries (HPAs) to electrical field stimulation (EFS) at $32 \mathrm{~Hz}$. The initial response $(n=7)$ in absence of any pharmacological agent (grey bar) was noted as a small vasoconstriction. Subsequently applied, exogenous noradrenaline (NA) markedly increased the vasoconstrictor effect of EFS ( $n=7)$. Guanethidine (Gua, $n=7$ ) and Tetrodotoxin (TTX, $n=7)$ reduced the effect of EFS in combination with exogenous NA $(n=6)$. BQ123 $(n=7)$ almost completely abolished the synergistic EFS and NA effect. Data are expressed as mean \pm SEM percentage change of the initial diameter.

$\# ; p \leq 0,05$ when compared to the initial response. *; $p \leq 0,05$ when compared to EFS in combination with exogenous NA.

\subsection{Rat middle cerebral arteries versus human pial arteries}

Comparing rat MCAs with HPAs, the CCRCs of both arteries showed that rat MCAs have significantly stronger contractile responses to the application of 5-HT (diameter decrease: $60.9 \pm 1.7 \%$ vs. $20.7 \pm 7.2 \%, p<0.001$ ) and ET-1 (diameter decrease: $70.8 \pm 1.6 \%$ vs. $40.6 \pm 9.5 \%, p=0.001$ ), but not to NA (diameter decrease: $12.9 \pm 2.1 \%$ vs. $11.5 \pm 4.8 \%, p=0.767)$. 
When applying EFS alone, no vasoconstriction was found in rat MCAs nor in HPAs. The combination of EFS and exogenous NA induced a diameter decrease in rat MCAs $\left(E_{\max }\right.$ $21.1 \pm 6.9 \%)$, that was significantly $(p=0.005)$ more pronounced in HPAs $(50.9 \pm 3.7 \%)$. The subsequent application of Gua did not significantly affect lumen diameter in rat MCAs $(1.2 \pm 7.8 \%)$, whereas a significant increase of lumen diameter was induced in HPAs $(24.3 \pm 6.3 \%)$. This difference in lumen diameter increase between rat MCAs and HPAs was found significant $(p=0.007)$.

The introduction of TTX on the synergistic vasoconstrictive effect of EFS and exogenous NA resulted in a lumen diameter increase of $12.3 \pm 5.2 \%$ in rat MCAs, whereas this increase was $37.6 \pm 2.8 \%$ in HPAs. This difference in lumen diameter increase was not significant $(p=0.077)$. The application of $B Q 123$ resulted in complete neutralization of the vasoconstriction induced by EFS and NA and was not significantly different between MCAs and HPAs $(p=0.178)$. The addition of Phen was only applied in rat MCAs since the number of HPA segments was limited and therefore not included in this comparison.

\subsection{Immunohistochemistry}

Pial artery cross-sections were immunohistochemically stained for ET-1. In these sections, the only clear location of ET-1 immunoreactivity is the endothelium (Figure 5.5).

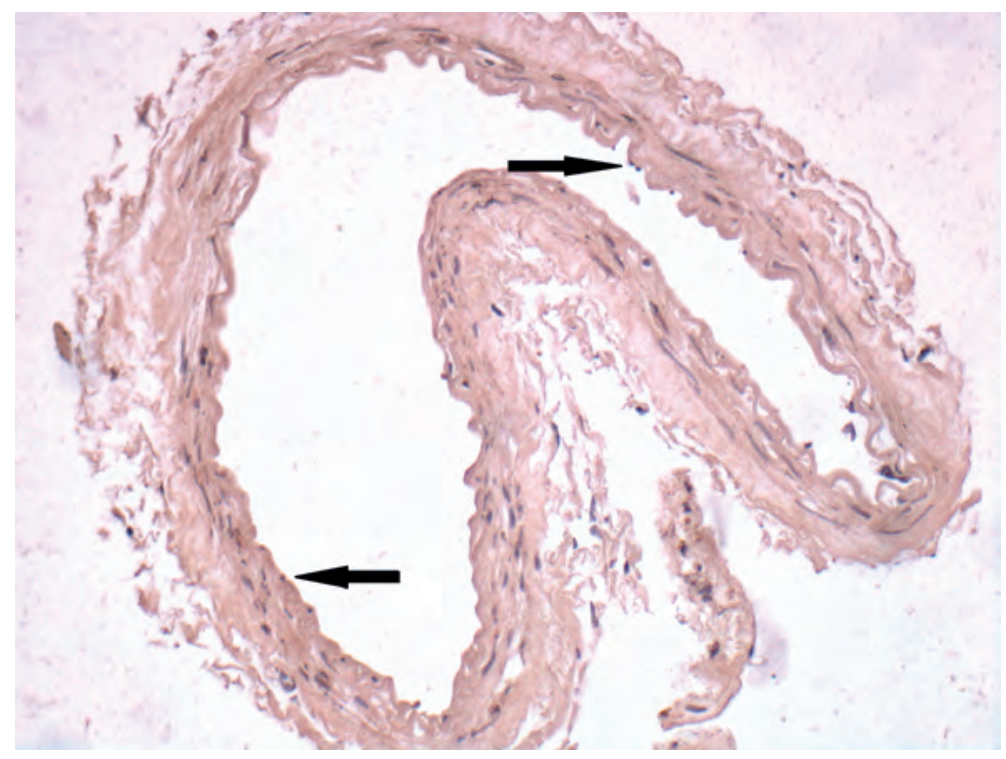

Figure 5.5 Image of a cross-section of a pressurized human pial artery following the immunohistochemical staining of ET-1. In this image, dark blue / brownish particles (as depicted by the arrows) were noted in the endothelium, whereas these were absent in a negative control, i.e. without a primary antibody, cross-section. Hence, ET-1 immunoreactivity is observed in the endothelium. 


\section{Discussion}

In this study, we have analyzed the regulation of cerebral artery vasomotor tone by studying the response of cerebral arteries to EFS in the absence and presence of several adrenergic modulators and ET-1 agonists and antagonists. We have shown that vasoconstriction of cerebral arteries following EFS requires the application of exogenous NA, whereas neither EFS nor exogenous NA alone induces a relevant vasoconstriction. The observed EFS + NA-induced vasoconstriction was counteracted by $\alpha$-adrenoreceptor antagonists as well as by a catecholamine-release inhibitor, blockade of the perivascular neurons using $\mathrm{TTX}$, and by the $\mathrm{ET}_{\mathrm{A}}$ receptor antagonist, $\mathrm{BQ} 123$. The latter suggests that ET-1 is involved in cerebral artery vasoconstriction.

\subsection{Adrenal and neuronal influences on vasoconstriction}

In contrast to ET-1 and 5-HT, cumulative concentrations of exogenous NA only caused limited vasoconstriction in rat and human cerebral arteries. Moreover, the application of EFS alone, simulating perivascular adrenergic nerve stimulation, did not induce vasoconstriction in HPAs and only a small, although significant, vasoconstriction in MCAs. Thus, the sole effect of perivascular sympathetic nerves on cerebral arteries is minimal. Our findings are in line with a recent review concluding that the role of NA released from perivascular sympathetic nerves on human cerebral arteries is minimal ${ }^{13}$. Nevertheless, adrenergic innervation of cerebral arteries has long been established and originates from the superior cervical ganglion ${ }^{3,13,15,20}$. Network density of these adrenergic nerve fibers decreases with increasing distal branching ${ }^{15,17,19-21}$. It has been described that adrenergic sensitivity of cerebral arteries is lower compared to peripheral arterial beds and that cumulative NA concentration induced contractions are smaller in cerebral arteries ${ }^{14,15,41}$. However, results on cerebral artery responses to EFS and NA vary widely ${ }^{13-16,19,42}$. Duckworth et al. and Aldasoro et al. reported on neurogenic and adrenergic vasoconstrictive influences on cerebral arteries. They found NA concentration-dependent, and EFS frequency-dependent, vasoconstriction in postmortem human cerebral arteries ${ }^{18,19}$. This is in contrast with our results and the results described by Bevan et al., who related these results to the marginal density of adrenergic innervation in $\mathrm{HPAs}^{43}$. Other explanations for the differences described in literature include artery caliber, species variability, and receptor type, density, and sensitivity ${ }^{13,15,17,19-21}$.

Remarkably, we found that the simultaneous application of exogenous NA and EFS caused a strong and significant vasoconstriction. When the adrenergic contribution was counteracted by Gua or Phen, or when the neurogenic contribution was counteracted by TTX, the induced vasoconstriction was partially to fully abolished. This suggests that a simultaneous and synergistic neurogenic and adrenergic effect is required for vasoconstriction of cerebral arteries. It has indeed been described that exogenous 
applied NA restored and potentiated EFS evoked vasoconstriction in rat tail and rabbit mesenteric arteries, but not in rabbit brachial, auricular and pulmonary arteries ${ }^{42,44}$. On the contrary, no additional effect or contradicting vasodilatation has also been reported following exogenous NA in addition to $\mathrm{EFS}^{45-47}$. In cerebral arteries, the synergistic vasoconstrictive effect of exogenous NA and EFS has not been reported on so far.

\subsection{A role for ET-1 in cerebral artery vasoconstriction}

A possible explanation for the required synergistic effect, is that exogenous NA application modulates the neurogenic or endothelial release of other neurotransmitters, which are subsequently activated by EFS. In this study, the involvement of ET-1 in this synergistic vasoconstrictive effect was evaluated. The application of the $\mathrm{ET}_{\mathrm{A}}$-receptor antagonist, $\mathrm{BQ123}$, completely counteracted the synergistic vasoconstrictive effect of EFS and exogenous NA. Moreover, cumulative concentrations of ET-1 caused a strong contractile response in both rat MCAs and HPAs. These results suggest an important contribution of ET-1 in cerebral artery vasoconstriction.

ET-1 is a potent vasoconstrictor and is predominantly synthesized by endothelial cells ${ }^{25,26}$. However, the synthesis and release of ET-1 by perivascular nerves of cerebral arteries has also been described ${ }^{27-29,32,48,49}$. These neurons primarily originate from the trigeminal ganglia, suggesting that sensory nerves are releasing ET-1, that may act as a neurotransmitter ${ }^{49-51}$.

In this study, ET-1 immunoreactivity was observed in the endothelium in cross-sections of HPAs. To assess whether ET-1 is also present within perivascular nerves, ET-1 immunoreactivity analysis in whole mount preparations is required, which we did not perform. A contribution of ET-1 from perivascular nerves could thus not be ruled out.

One could hypothesize ET-1 release from perivascular nerves since vasoconstriction is reversed by Phen and TTX. The latter substance abolishes perivascular innervation, possibly including sensory nerve innervation. However, another plausible explanation for the abolishing effect of TTX is that the combined effect of EFS and NA is disturbed by counteracting neurogenic control. The abolishing effect of Phen could also point toward a neuronal release of ET-1 by antagonizing presynaptic $\alpha_{2}$-receptors on perivascular sensory nerves. However, in both these suggestions, the release of endothelium-derived ET-1 by NA is still possible and was not further assessed.

In the internal mammary artery, EFS evoked vasoconstriction was found to be diminished following the removal of endothelium ${ }^{12}$. Furthermore, vasoconstriction following EFS was reduced after application of bosentan, a non-selective ET-receptor antagonist, in endothelial-intact arteries but not in denuded arteries. Moreover, a rightward shift of phenylephrine sensitivity in distal mesenteric branches in the presence of bosentan was described by Hilgers et al. ${ }^{10}$. Based on these studies, it is suggested that neurogenic evoked vasoconstriction requires an endothelium-derived vasoconstrictor, and that ET-1 may contribute to this circuitry. 
The importance of ET-1 induced vasoconstriction by the $\mathrm{ET}_{\mathrm{A}}$-receptor in the cerebrovascular bed has earlier been suggested by several studies ${ }^{29,32,34,35,49,52,53}$. Exogenous concentration-dependent ET-1 induced vasoconstriction has been described in human pial arteries, and in rat basilar and pial arteries ${ }^{29,32-34}$. Moreover, the $\mathrm{ET}_{\mathrm{A}^{-}}$ receptor antagonists, BQ123 and PD156707, have been evaluated following vasoconstriction of human pial arteries and rat basilar artery, resulting in the reversal of this vasoconstriction ${ }^{29,32,35,54}$. This is in line with our results and supports the hypothesis of an ET-1 contribution in cerebral artery vasoconstriction.

Based on our results, simultaneous neurogenic and adrenergic activation is required for cerebral artery vasoconstriction. We hypothesize that NA modulates the release of ET-1 in this vasoconstriction. The release of ET-1 might be either endothelial or neuronal, or both. Upon release, $\mathrm{ET}-1$ binds to the $\mathrm{ET}_{\mathrm{A}}$-receptor on smooth muscle cells contributing to cerebral artery vasoconstriction. To the best of our knowledge, an interaction between NA and ET-1 in cerebral arteries has not been reported on. This combined effect of EFS and NA and the modulatory role of NA on ET-1 is illustrated in Figure 5.6.

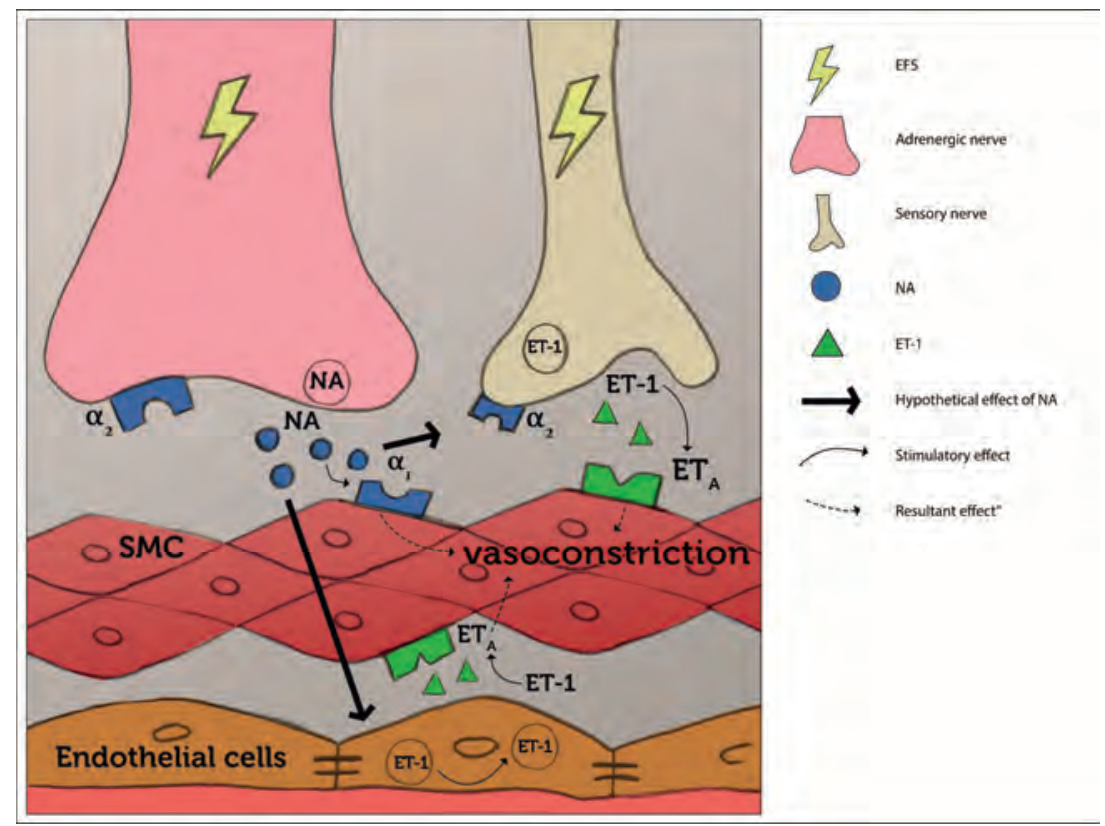

Figure 5.6 Illustration of how NA may modulate the vasoconstrictor responses to nerve stimulation via ET-1 in cerebral arteries. As suggested by our findings, NA acting on smooth muscle cells (SMCs) a1-receptors has a modest vasoconstrictive effect. Yet, in conjunction with EFS, NA may activate the presynaptic sensory nerve a2-receptor resulting in ET-1 release. In addition, or alternatively, when EFS is applied together with exogenous NA, exogenous NA may stimulate the release of endothelial ET-1. The released ET-1 induces a strong vasoconstriction via the ETAreceptor located on SMCs. How NA potentiates ET-1 and the source of ET-1, either released by perivascular nerves or by the endothelium, remains to be established. 


\subsection{Differences between rat MCAs and HPAs}

When comparing the sensitivity of rat MCAs and HPAs to cumulative concentrations of vasoactive substances, we found that ET-1 and 5-HT were more potent in rat MCAs than in HPAs, yet NA did not elicit a contractile response in rat MCAs, nor in HPAs.

However, a contractile response was observed when the application of NA was accompanied by EFS. Since the baseline contractile response to the simultaneous application of EFS and NA varied highly between rat MCAs and HPAs, responses to subsequently applied vasoactive substances, i.e. Gua, TTX and BQ123, are statistically difficult to compare. This variation in baseline measurement was best taken into account using ANCOVA analysis. Still, based on the combination of high variation in baseline measurements, a statistical comparison should be interpreted cautiously.

Regarding the subsequent responses, we found no significant differences between rat MCAs and HPAs following the addition of TTX or BQ123. In contrast, the application of Gua did affect the EFS and NA induced vasoconstriction in HPAs, but not in rat MCAs.

The differences between rat MCAs and HPAs are most likely due to specie and/or artery type differences. Variation in arterial vasoconstrictive and vasodilatory responses between rat and human arteries have previously been reported ${ }^{55,56}$. Moreover, middle cerebral arteries are first order cerebral arteries, whereas pial arteries are last order arteries. Reported differences between these types of cerebral arteries include variation in adventitial nerve and receptor type, density, distribution, and sensitivity $^{15,17,20}$. In addition, an anesthesia effect should be considered, as the HPAs were derived from patients who underwent surgery for epilepsy. Medication used for induction and maintenance of anesthesia are known to affect arterial contractile responses ${ }^{57-59}$. Finally, an explanation for the observed differences in contractile responses may relate to the underlying disorder, i.e. drug-resistant epilepsy, in the human samples. Abnormal contractile responses have previously been reported in epilepsy patients and may be related to the pathophysiology of epilepsy or to antiepileptic drugs $^{60-63}$.

\subsection{Limitations and future directions}

The number of human pial arterial experiments were restricted due to the limited number of patients that undergo epilepsy surgery. Therefore, we have evaluated experiments with Phen only in rat MCAs. The lack of Phen experiments in human cerebral arteries limits the comparison between rat MCAs and HPAs. However, the application of Phen was only included to confirm that EFS alone does not result in vasoconstriction, as it counteracts NA. We found that the application of EFS alone showed a limited diameter decrease in rat MCAs and HPAs. Nevertheless, future studies should include the application of Phen in HPAs, and experiments in the presence and absence of selective $\alpha_{1}$ - and $\alpha_{2}$-receptor antagonists. 
In this study, we have only evaluated the involvement of ET-1 in cerebral arteries based on the study of Pagán et al. The possible release of co-transmitters like neuropeptide-Y, adenosine triphosphate, or serotonin was not assessed. Also, the interaction with other perivascular nerve fibers like parasympathetic, sensory, or non-adrenergic noncholinergic nerves was not evaluated. The influence of sensory nerves, expressing ET-1, is of particular interest and could be assessed by the application of capsaicin.

The role of endothelial vasomotor control by NO and ET-1 was not assessed in this study. Endothelial denudation of cerebral arteries could be an interesting next study to assess whether ET-1 release is of endothelial or neuronal origin, and to assess the contribution of NO including its reciprocal interaction with ET-1.

In this study, functional pharmacological experiments were performed. Extensive immunohistochemistry to localize and quantify neurons, specific neurotransmitters, and receptor expression was not included. These localization and quantification experiments, and further experiments involving the selective adrenergic receptor influence, the endothelial influence, the co-release of other vasoconstrictive neurotransmitters, and the interaction with other perivascular nerve fibers, are interesting and important topics for future experiments.

In this study, we have compared cerebral arteries from epilepsy patients (HPAs) to rat MCAs. The differences found between the two groups may be related to various dissimilarities between the two groups. Many of these dissimilarities can be bypassed by a preclinical study comparing epilepsy versus control animals. In this way, similar arteries of similar species are assessed under comparable conditions. Such a study may address differences between cerebral artery vasoconstriction under physiological and epilepsy-related conditions.

\section{Conclusion}

Our results demonstrate that cerebral artery vasoconstriction depends on the simultaneous action of neurogenic stimulation and adrenergic modulation. We hypothesize that NA modulates the release of ET-1 by perivascular sensory nerves and/or endothelial cells. The released ET-1 subsequently binds to the $\mathrm{ET}_{\mathrm{A}}$-receptor on smooth muscle cells resulting in vasoconstriction. Further experiments are required to elucidate the mechanisms by which NA modulates ET-1 and to assess the role of other co-transmitters. 


\section{References}

1. Lok J, Gupta P, Guo S, et al. Cell-cell signaling in the neurovascular unit. Neurochem Res. 2007;32: 2032-45.

2. Longden TA, Nelson MT. The control of cerebral blood flow. 2016;22:183-96.

3. Hamel E. Perivascular nerves and the regulation of cerebrovascular tone. J Appl Physiol. 2006;100: 1059-64.

4. Bagher $P$, Segal SS. Regulation of blood flow in the microcirculation: role of conducted vasodilation. Acta Physiol. (Oxf). 2011;202:271-84.

5. Peterson EC, Wang Z, Britz G. Regulation of cerebral blood flow. Int J Vasc Med. 2011;2011.

6. Muñoz MF, Puebla M, Figueroa XF. Control of the neurovascular coupling by nitric oxide-dependent regulation of astrocytic $\mathrm{Ca}(2+)$ signaling. Front Cell Neurosci. 2015;9:59.

7. Iadecola C. Neurovascular regulation in the normal brain and in Alzheimer's disease. Nat Rev Neurosci. 2004;5:347-60.

8. Westcott EB, Segal SS. Perivascular Innervation: A multiplicity of roles in vasomotor control and myoendothelial signaling. Microcirculation. 2013;20:217-38.

9. Nausch LWM, Bonev a. D, Heppner TJ, Tallini Y, Kotlikoff MI, Nelson MT. Sympathetic nerve stimulation induces local endothelial Ca2+ signals to oppose vasoconstriction of mouse mesenteric arteries. Am J Physiol Hear Circ Physiol 2012;302:H594-602.

10. Hilgers RHP, De Mey JGR. Myoendothelial coupling in the mesenteric arterial bed; segmental differences and interplay between nitric oxide and endothelin-1. Br J Pharmacol. 2009;156:1239-47.

11. Van Riper DA, Bevan JA. Evidence that neuropeptide $Y$ and norepinephrine mediate electrical fieldstimulated vasoconstriction of rabbit middle cerebral artery. Circ Res. 1991;68:568-77.

12. Pagán RM, Martínez AC, Hernández M, et al. Endothelial and neural factors functionally involved in the modulation of noradrenergic vasoconstriction in healthy pig internal mammary artery. Biochem Pharmacol. 2012;83:882-92.

13. Lee TJF, Chang $\mathrm{HH}$, Lee $\mathrm{HC}$, et al. Axo-axonal interaction in autonomic regulation of the cerebral circulation. Acta Physiol. (Oxf). 2011;203:25-35.

14. Duckles SP, Bevan JA. Pharmacological characterization of adrenergic receptors of a rabbit cerebral artery in vitro. J Pharmacol Exp Ther. 1976;197:371-8.

15. Bevan RD, Dodge J, Nichols P, et al. Weakness of sympathetic neural control of human pial compared with superficial temporal arteries reflects low innervation density and poor sympathetic responsiveness. Stroke. 1998;29:212-21.

16. Van Riper DA, Bevan JA. Selective variation of agonist and neurally mediated vasoconstriction with rabbit middle cerebral artery branch order. J Pharmacol Exp Ther. 1991;257:879-86.

17. Handa $\mathrm{Y}$, Caner H, Hayashi M, Tamamaki N, Nojyo Y. The distribution pattern of the sympathetic nerve fibers to the cerebral arterial system in rat as revealed by anterograde labeling with WGA-HRP. Exp Brain Res. 1990;82:493-8.

18. Aldasoro M, Martínez C, Vila JM, Medina P, Lluch S. Influence of endothelial nitric oxide on adrenergic contractile responses of human cerebral arteries. J Cereb Blood Flow Metab. 1996;16:623-8.

19. Duckworth JW, Wellman GC, Walters CL, Bevan JA. Aminergic histofluorescence and contractile responses to transmural electrical field stimulation and norepinephrine of human middle cerebral arteries obtained promptly after death. Circ Res. 1989;65:316-24.

20. Cipolla MJ, Li R, Vitullo L. Perivascular innervation of penetrating brain parenchymal arterioles. J Cardiovasc Pharmacol. 2004;44:1-8.

21. Dodge JT, Bevan RD, Bevan JA. Comparison of density of sympathetic varicosities and their closeness to smooth muscle cells in rabbit middle cerebral and ear arteries and their branches. Circ Res. 1994;75: 916-25.

22. Mccarron RM, Chen Y, Tomori T, et al. Endothelial-mediated regulation of cerebral microcirculation. J. Physiol. Pharmacol. 2006;57:133-44.

23. Dora K a. Coordination of vasomotor responses by the endothelium. Circ. J. 2010;74:226-32.

24. Andresen J, Shafi NI, Bryan RM. Endothelial influences on cerebrovascular tone. J Appl Physiol. 2006; 100:318-27.

25. Thorin E, Webb DJ. Endothelium-derived endothelin-1. Pflugers Arch Eur J Physiol. 2010;459:951-8. 
26. Khimji A, Rockey DC. Endothelin-Biology and disease. Cell Signal. Elsevier B.V.; 2010;22:1615-25.

27. Loesch A, Burnstock G. Endothelin in human cerebrovascular nerves. 404S Clin. Sci. 2002;103:404-7.

28. Loesch A, Milner P, Burnstock G. Endothelin in perivascular nerves. An electron-immunocytochemical study of rat basilar artery. Neuroreport. 1998;9:3903-6.

29. Compeer MG, Janssen GMJ, De Mey JGR. Endothelin-1 and endothelin-2 initiate and maintain contractile responses by different mechanisms in rat mesenteric and cerebral arteries. Br J Pharmacol. 2013;170:1199-209.

30. Khimji A, Rockey DC. Endothelin-Biology and disease. Cell Signal. 2010;22:1615-25.

31. Reijerkerk A, Lakeman $\mathrm{K}$ a M, Drexhage $\mathrm{J}$ a $\mathrm{R}$, et al. Brain endothelial barrier passage by monocytes is controlled by the endothelin system. J Neurochem. 2012;121:7307.

32. Pierre LN, Davenport a P. Blockade and reversal of endothelin-induced constriction in pial arteries from human brain. Stroke. 1999;30:638-43.

33. de Aguilera EM, Irurzun a, Vila JM, Aldasoro M, Galeote MS, Lluch S. Role of endothelium and calcium channels in endothelin-induced contraction of human cerebral arteries. Br J Pharmacol. 1990;99: 439-40.

34. Faraci FM. Effects of endothelin and vasopressin on cerebral blood vessels [Internet]. Am J Physiol. 1989:H799-803.

35. Thorin E, Nguyen TD, Bouthillier A. Control of vascular tone by endogenous endothelin-1 in human pial arteries. Stroke. 1998;29:175-80.

36. Hardebo JE, Hanko J, Kåhrstrom J, Owman C. Electrical field stimulation in cerebral and peripheral arteries: a critical evaluation of the contractile response. J Auton Pharmacol. 1986;6:85-96.

37. WHO | Epilepsy. WHO. World Health Organization; 2016;

38. Zhao M, Nguyen J, Ma H, Nishimura N, Schaffer CB, Schwartz TH. Preictal and ictal neurovascular and metabolic coupling surrounding a seizure focus. J Neurosci. 2011;31:13292-300.

39. Parfenova H, Carratu P, Tcheranova D, Fedinec A, Pourcyrous M, Leffler CW. Epileptic seizures cause extended postictal cerebral vascular dysfunction that is prevented by HO-1 overexpression. AJP Hear Circ Physiol. 2005;288:H2843-50.

40. Heinemann U, Kaufer D, Friedman A. Blood Brain Barrier Dysfunction, TGFb signaling and AStrocyre Dysfunction in Epilepsy. Glia. 2012;60:1251-7.

41. Longo LD, Ueno N, Zhao Y, Zhang L, Pearce WJ. NE-induced contraction, alpha 1-adrenergic receptors, and Ins(1,4,5)P3 responses in cerebral arteries. Am J Physiol. 1996;270:H915-23.

42. Karachentseva O V, Yartsev VN, Dvoretskii DP. Noradrenaline can restore decreased neurogenic vasoreactivity. Neurosci Behav Physiol. 2009;39:161-6.

43. Bevan R, Dodge J, Nichols $P$, et al. Responsiveness of human infant cerebral arteries to sympathetic nerve stimulation and vasoactive agents. Pediatr Res. 1998;44:730-9.

44. Su C. Potentiative effects of alpha agonistic sympathomimetic amines on vasoconstriction by adrenergic nerve stimulation. J Pharmacol Exp Ther. 1980;215:377-81.

45. Van Riper DA, Bevan JA. Electrical field stimulation-mediated relaxation of rabbit middle cerebral artery. Evidence of a cholinergic endothelium-dependent component. Circ Res. 1992;70:1104-12.

46. Ebeigbe $A B$, Gantzos RD, Webb RC. Relaxation of rat tail artery to electrical stimulation. Life Sci. 1983;33:303-9.

47. Atkinson J, Boillat N, Fouda AK, Guillain H, Sautel M, Sonnay M. Noradrenaline inhibits vasoconstriction induced by electrical stimulation. Gen Pharmacol. 1987;18:219-23.

48. Loesch A. Perivascular nerves and vascular endothelium: Recent advances. Histol. Histopathol. 2002;17:591-7.

49. Dashwood MR, Loesch A. Endothelin-1 as a neuropeptide: Neurotransmitter or neurovascular effects? J Cell Commun Signal. 2010;4:51-62.

50. Milner P, Loesch A, Burnstock G. Endothelin immunoreactivity and mRNA expression in sensory and sympathetic neurones following selective denervation. Int J Dev Neurosci. 2000;18:727-34.

51. Milner P, Loesch A, Burnstock G. Neural endothelin in hypertension: increased expression in ganglia and nerves to cerebral arteries of the spontaneously hypertensive rat. J Vasc Res. 2000;37:39-49.

52. Loesch A, Dashwood MR, Coppi AA. Immunoreactive endothelin-1 and endothelin a receptor in basilar artery perivascular nerves of young and adult capybaras. Cells Tissues Organs 2013;198:47-56.

53. Hardebo JE, Kahrstrom J, Owman C, Salford LG. Endothelin is a potent constrictor of human intracranial arteries and veins. Blood Vessels 1989;26:249-53. 
54. Nilsson T, Cantera L, Adner M, Edvinsson L. Presence of contractile endothelin-A and dilatory endothelin-B receptors in human cerebral arteries. Neurosurgery. 1997;40:346-53.

55. Grände G, Nilsson E, Edvinsson L. Comparison of responses to vasoactive drugs in human and rat cerebral arteries using myography and pressurized cerebral artery method. Cephalalgia. 2013;33:152-9.

56. Bialecki RA, Fisher CS, Murdoch WW, Barthlow HG, Bertelsen DL. Functional comparison of endothelin receptors in human and rat pulmonary artery smooth muscle. Am J Physiol. 1997;272:L211-8.

57. Hama-Tomioka K, Kinoshita H, Azma T, et al. The role of 20-hydroxyeicosatetraenoic acid in cerebral arteriolar constriction and the inhibitory effect of propofol. Anesth Analg. 2009;109:1935-42.

58. Biddle NL, Gelb AW, Hamilton JT. Propofol differentially attenuates the responses to exogenous and endogenous norepinephrine in the isolated rat femoral artery in vitro. Anesth Analg. 1995;80:793-9.

59. Marin J, Recio L, Reviriego J, Sanchez-Ferrer CF, Salaices M. Effects of morphine on noradrenaline release from cerebral and peripheral vascular smooth muscle. Gen Pharmacol. 1986;17:705-8.

60. Ma H, Zhao M, Schwartz TH. Dynamic neurovascular coupling and uncoupling during ictal onset, propagation, and termination revealed by simultaneous in vivo optical imaging of neural activity and local blood volume. Cereb Cortex. 2013;23(4):885-99.

61. Seçkin H, Yigitkanli K, Besalti O, et al. Lamotrigine attenuates cerebral vasospasm after experimental subarachnoid hemorrhage in rabbits. Surg Neurol. 2008;70:344-51.

62. Wang $\mathrm{H}, \mathrm{Gao}$ J, Lassiter TF, et al. Levetiracetam is neuroprotective in murine models of closed head injury and subarachnoid hemorrhage. Neurocrit Care. 2006;5:85-92.

63. Chuang $\mathrm{Y}-\mathrm{C}$, Chuang $\mathrm{H}-\mathrm{Y}$, Lin T-K, et al. Effects of long-term antiepileptic drug monotherapy on vascular risk factors and atherosclerosis. Epilepsia. 2012;53:120-8. 



\section{Chapter

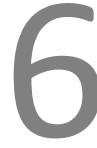

Lipofuscin in the cerebral vascular wall: a technical

and explorative analysis

K. Hakvoort, L.A.M. Otto, R.H.L. Haeren, G. Hoogland, O. Schijns, H. Vink, D. Klein, J.J. van Overbeeke, M.A.M.J. van Zandvoort, K. Rijkers 


\section{Abstract}

\section{Introduction}

Vascular oxidative stress is a common denominator in the pathophysiology of several neurodegenerative diseases including epilepsy. Lipofuscin is a non-degradable end-product of oxidative stress and therefore a potential marker for the total amount of experienced oxidative stress. We have observed prominent autofluorescent particles in the pial arterial wall of drugresistant epilepsy patients who underwent resective brain surgery and hypothesize that these particles represent lipofuscin. The aim of this study is to characterize and quantify these particles.

\section{Methods}

Six samples of neocortex were obtained from operated epilepsy patients and three non-epileptic control samples were obtained intraoperatively or post-mortem. Fluorescence spectroscopy and fluorescence lifetime imaging were performed to characterize fluorescent properties. Adjacent histological slices were stained for cross-reference. Using two-photon laser scanning microscopy, particle volume and density were assessed.

\section{Results}

Autofluorescent particles were noted within the cerebral vascular wall of both patients and controls. An emission spectrum between 420 to $720 \mathrm{~nm}$ with a maximum of 580 to $600 \mathrm{~nm}$, and an autofluorescence lifetime of 1.41 to 1.55 +/- ns was found. These specific fluorescent properties characterize lipofuscin. Using adequate software, particle density and size analyses were performed on the two-photon images, revealing a variation of particle density and size between the two groups.

\section{Conclusion}

We have demonstrated the presence of lipofuscin in the cerebral vascular wall. Analysis of lipofuscin could be a new method to detect and quantify oxidative stress. An epilepsy-related increase of lipofuscin within the vascular wall reflecting increased oxidative stress and vascular dysfunction, needs to be assessed. 


\section{Introduction}

Low concentrations of reactive oxygen species contribute to cell processes. At higher concentrations, they can damage the cell through peroxidation ${ }^{1}$. The formation of reactive oxygen species occurs during aerobic metabolism, in which mitochondria are responsible for adenosine triphosphate (ATP) production. Oxidative stress applies to a condition of high metabolic activity, in which there is excessive generation of reactive oxygen species that cannot be counterbalanced by the antioxidant defense system, resulting in structural cell damage $\mathrm{e}^{1-3}$. Mitochondrial dysfunction directly leads to oxidative stress, which on itself induces mitochondrial damage ${ }^{4,5}$, thus creating a positive feedback loop.

During oxidative stress, reactive oxygen species facilitate aldehyde-mediated crosslinking of macro-molecules while they are being degraded inside lysosomes, forming a non-degradable substance called lipofuscin ${ }^{5,6}$. Hence, lipofuscin is an end-product of oxidative stress. Lipofuscin is composed of 'waste-material' from cells and contains lipids, proteins, carbohydrates, and metals ${ }^{5,7}$. Microscopically, lipofuscin can be distinguished from other lipopigments by its specific autofluorescent properties ${ }^{5,8,9}$. Since mitotic cells are able to discard lipofuscin through dilution, accumulation of lipofuscin mainly occurs in post-mitotic cells. Due to its non-degradable nature, lipofuscin accumulates with age, and is often referred to as 'age pigment' ${ }^{10}$. As a nondegradable end-product of oxidative stress, lipofuscin could be a potential marker for the total amount of experienced oxidative stress.

The brain is particularly receptive to oxidative stress due to the lack of energy storage capacity, high oxygen demand, and limited antioxidant defense mechanisms ${ }^{11-13}$. As a result, oxidative stress contributes to the pathophysiology of several neurodegenerative diseases including Alzheimer's disease, Parkinson's disease, and epilepsy ${ }^{14}$. Important features of neurodegeneration include microvascular dysfunction, increased blood-brain barrier permeability, angiogenesis, and dysfunctional neurovascular coupling ${ }^{15-21}$. A common denominator in these vascular pathophysiological processes is oxidative stress ${ }^{11}$. Vascular abnormalities contribute to the pathophysiology of neurodegenerative diseases and lead to additional oxidative stress, reinforcing each other in a positive feedback loop ${ }^{15,22}$. Moreover, mitochondrial dysfunction, which is extensively reported in this regard, further contributes to increased oxidative stress ${ }^{11,14,23-25}$. This increased oxidative stress could be reflected by the accumulation of lipofuscin within vascular structures of these patients.

In a pilot study using two-photon laser scanning microscopy, we observed prominent autofluorescent particles in the pial artery wall of drug-resistant epilepsy patients who underwent resective brain surgery. We hypothesize these particles to represent lipofuscin. In the present study, we aim to characterize and quantify these autofluorescent particles. 


\section{Materials and methods}

\subsection{Study population}

Cortical brain samples of six epilepsy patients (E1-E6, further referred to as patients), who underwent resective surgery as treatment for drug-resistant epilepsy, were collected intraoperatively. Epilepsy-negative controls, further referred to as controls, consisted of one human brain sample collected during surgery for a glial tumour (C1) and two post-mortem brain tissue samples from humans with no known neurological disorder or brain pathology (C2-C3). Demographic characteristics of patients and controls are summarized in Table 6.1.

Resected brain tissue (E1-E6 and C1) was partly used for routine histopathological evaluation. Remaining tissue, that otherwise would have been discarded, was included for this study. Although no medical ethical approval was lawfully required, a signed informed consent was obtained preoperatively in all cases. Experimental protocols of human tissue-handling were in accordance with Good Clinical Practice guidelines.

\subsection{Tissue processing}

Intraoperatively collected brain tissue samples of all patients (E1-E6) included anterior temporal lobe neocortex, and parieto-temporal neocortex in the control (C1). One part of the tissue samples was immediately stored in HEPES-buffered physiologic salt solution (HEPES-PSS). HEPES-PSS was continuously maintained at a temperature of $37^{\circ} \mathrm{C}$, and contained in $\mathrm{mM}: \mathrm{KCl} 4.7, \mathrm{NaCl} 144, \mathrm{CaCl}_{2} 2.5, \mathrm{KH}_{2} \mathrm{PO}_{4} 1.2, \mathrm{MgSO}_{4} 1.2, \mathrm{HEPES}$ 14.9, and glucose 5.5, at pH 7.4. Subsequently, pial arteries of E1-E4 and C1 were isolated by microdissection, after which experiments were performed within two to three hours after resection.

Another part of the tissue was fixated in $4 \%$ paraformaldehyde for 48 hours, and subsequently embedded in paraffin and sectioned at a thickness of $5 \mu \mathrm{m}$. Post-mortem material was obtained within $72 \mathrm{~h}$ after establishment of death and processed in the same way. In one patient (E1), an additional part of the collected tissue was stored as fresh-frozen tissue at minus $80^{\circ} \mathrm{C}$ directly following resection, and sectioned at a thickness of $12 \mu \mathrm{m}$.

\subsection{Visualization of autofluorescent particles in isolated pial arteries}

Isolated pial arteries (E1-E4 and C1) with an average length of 2-3 mm were mounted between two glass micropipettes (100-125 $\mu \mathrm{m}$ ) of an arteriograph organ chamber (Living Systems Instrumentation, Burlington, VT, USA). After flushing residual luminal blood out, the arterial vascular wall was visualized with two-photon laser scanning microscopy, using a Leica TCS SP5 (Leica Microsystems GmbH, Wetzlar, Germany). A Ti:Sapphire Chameleon Ultra II (Coherent, USA) mode locked at $820 \mathrm{~nm}$ was used as the excitation source. A Leica HCX APO L 20x/1.00 objective was used for excitation and 
epicollection. Three colour-coded channels were used for detection, namely 399-499 $\mathrm{nm}$ (blue), 509-552 nm (green), and 568-650 nm (red).

Thereafter, two-photon laser scanning microscopy image stacks were included for quantitative analysis of the autofluorescent particles. Quantification was performed with Imaris@ image-processing software (version 8.3, Bitplane AG, Zürich) providing a three-dimensional (3D) reconstruction of the stacks. Using this technique, sampling errors due to possible heterogenous distribution of autofluorescent particles are avoided, in contrast to quantification of autofluorescent particles on tissue sections. Furthermore, the 3D representations allow a spatial visualization of particle distribution and volume measurements.

Fluorescent particles were defined by their threshold, which was determined based on intensity in relation to background signal and local contrast. Optimal threshold levels were set separately for each stack, following inter-observer agreement of two independent observers [LO \& DK]. Validation of the segmentation was performed by superimposing the reconstructed autofluorescent particles on the original confocal z-stack. Subsequently, the red channel was selected to calculate the number of particles, particle density, and diameter distribution. Finally, volume reconstruction was performed on the three-dimensional image of the stack in the red channel to determine mean particle volume and particle volume density.

\subsection{Fluorescence spectroscopy}

Unstained paraffin sections of neocortical parenchyma of four patients (E1, E2, E5, E6) and two controls (C2, C3) were used for autofluorescence spectrum analysis. A Leica DM4000 B LED microscope (Leica Microsystems GmbH, Wetzlar, Germany) was used with HC PLAN APO $20 \times / 0.70$ and HC PLAN APO 40x/0.75 water objectives, and an HXC PLAN APO 100×/1.40-0.70 oil immersion objective. Images were recorded using a Nuance Multispectral Imaging System (PerkinElmer, Hopkinton, MA, USA) with version 3.0.2 software.

Nuance microscopy registers the spectrum of light emitted by a specimen when excited in a narrow wavelength range. We applied excitation wavelengths between 420 and $460 \mathrm{~nm}$, while emission was evaluated through a long pass filter, passing all fluorescence above $420 \mathrm{~nm}$. The spectrum, obtained in ascending steps of $20 \mathrm{~nm}$, was determined from several autofluorescent particles located in both the vascular wall and the cortical parenchyma. Subsequently, multispectral analysis was performed by "unmixing" the generated spectral curves of the various fluorescent materials, thereby differentiating the spectrum of the particles of interest from the spectrum of other (background) structures. To assess potential influence of formaldehyde and/or paraffin on the spectrum, the analysis was repeated on fresh-frozen tissue once (E1). 


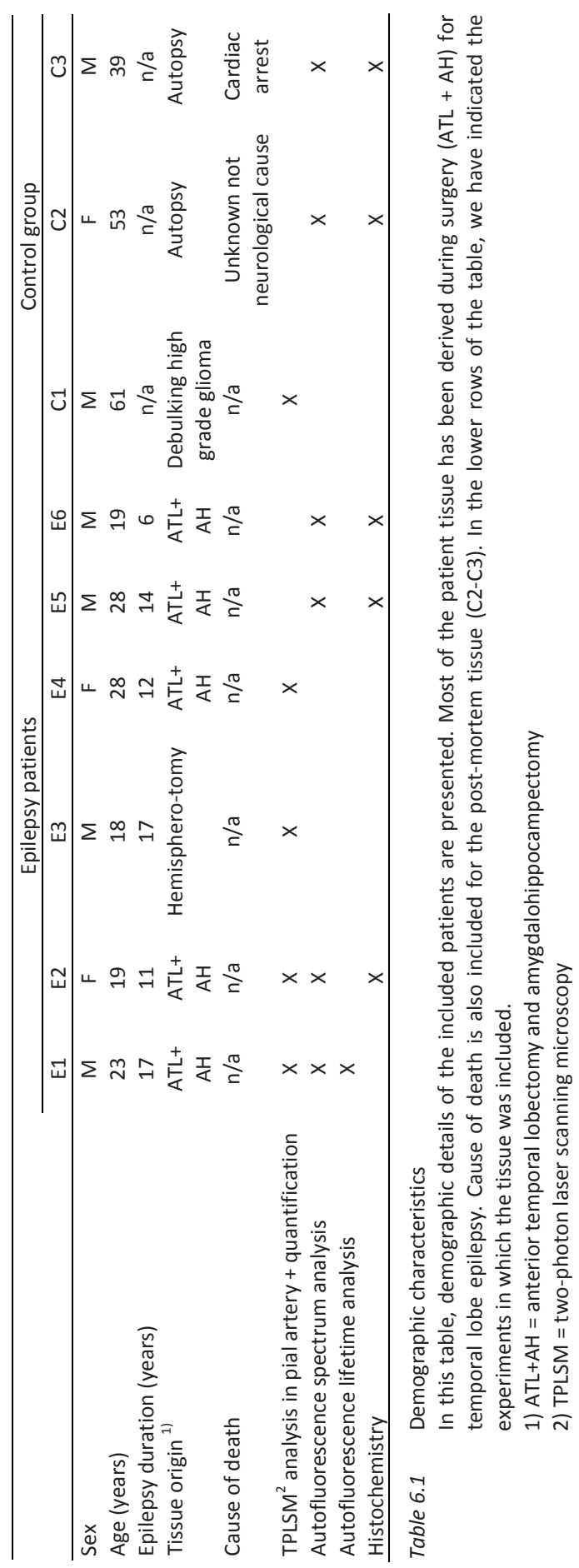




\subsection{Fluorescence lifetime imaging}

Additional autofluorescent characteristics of the particles were obtained by performing lifetime analysis using fluorescence lifetime imaging measurement. First, autofluorescence spectrum measurement was repeated to verify the analysis of similar autofluorescent particles, using a Leica TCS SP5 two-photon laser scanning microscope (Microsystems $\mathrm{GmbH}$, Wetzlar, Germany) with the aforementioned settings.

Fluorescence lifetime imaging was then performed with a Becker \& Hickl time correlated single photon counting module (SPC 830, Becker \& Hickl, Berlin, Germany). Four descanned colour-coded PMT detectors were used for detection, namely 400-420 nm (blue), 429-513 nm (green) and 518-646 nm (red).

Obtained lifetime images were analyzed using a bi-exponential decay curve, out of which the mean lifetime was calculated using the following formula:

$\tau_{\text {mean }}=\left(\alpha_{1} \bullet \tau_{1}^{2}+\alpha_{2} \bullet \tau_{2}^{2}\right) /\left(\alpha_{1} \bullet \tau_{1}+\alpha_{2} \bullet \tau_{2}\right)^{26}$.

\subsection{Histochemistry}

Periodic acid Schiff's (PAS) staining was performed on neocortical slides adjacent to the slides used for fluorescence microscopy of patients (E2, E5, E6) and controls (C2, C3). This staining was selected as cross-reference for the detection of lipofuscin, as Schiff's reagent detects polysaccharides present in lipofuscin resulting in a pronounced pinkred color. However, this staining is not lipofuscin-specific since it also stains glycogen and lipid droplets.

Neocortical slides were first deparaffinized and treated with periodic acid $0.5 \%$ solution for 5 minutes, then rinsed with distilled water. Subsequently, slides were put in Schiff's reagent for 15 minutes and washed in tap water for 5 minutes. Hereafter, the slides were mounted and analysed with light microscopy (BX51TF, Olympus) with bright-field illumination on 20 and 40 times magnification.

\section{Results}

\subsection{Visualization of autofluorescent particles in pial artery}

An abundant number of autofluorescent particles was noted within isolated pial arteries of both groups, using two-photon laser scanning microscopy (Figure 6.1). Image stacks of one patient (E1) were excluded due to poor image quality because of overexposure of excitation light. 


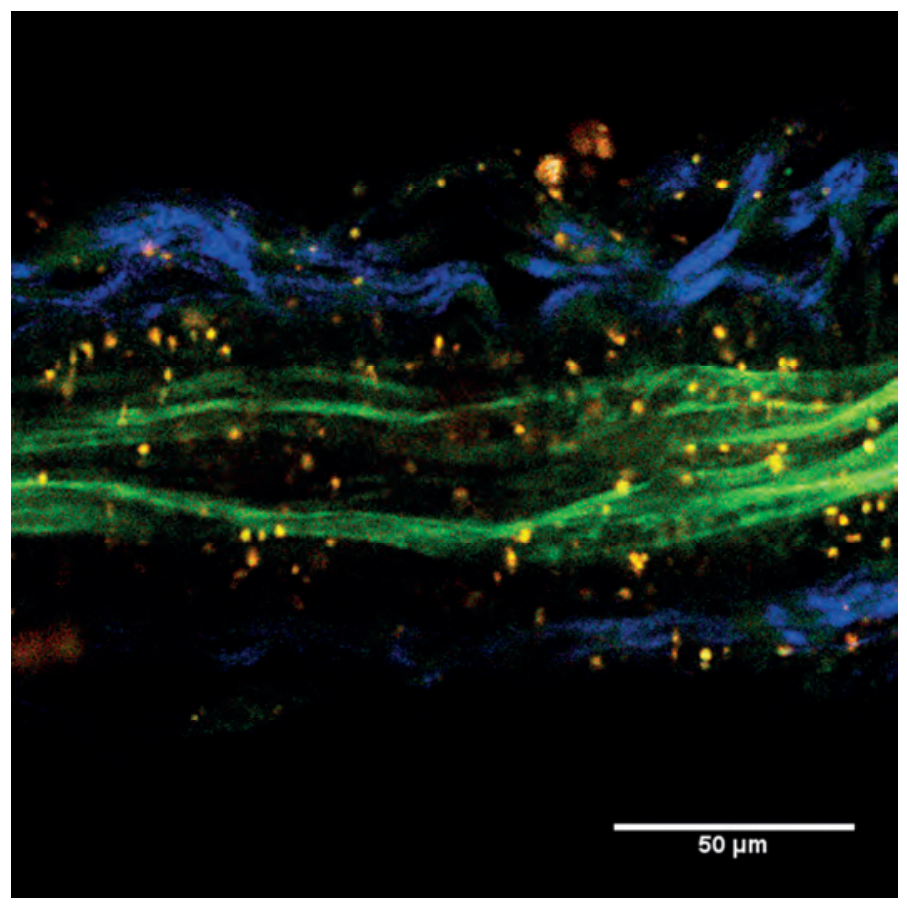

Figure 6.1 Representative two-photon laser scanning microscopy longitudinal cross-section of pial artery. The wave-like blue bands reflect collagen within the adventitia, while the external lamina is visible in green. The remarkable yellowish particles found throughout the vascular wall are the autofluorescent particles of interest in this study.

In Figure 6.2, a representative spherical 3D-reconstruction of particles in the vessel wall is shown. Such reconstructions allow localization of particles surrounding the external elastic membrane. Particles were mainly located within the adventitia, and to a lesser extent in the tunica media. Comparing the patients and control, mean particle diameter, volume, and density in patient E2 was more or less comparable to the control. In patients E3 and E4 mean particle diameter was smaller and volume per particle was less compared to the control, whereas particle density was higher (Table 6.2 and Figure 6.3). 


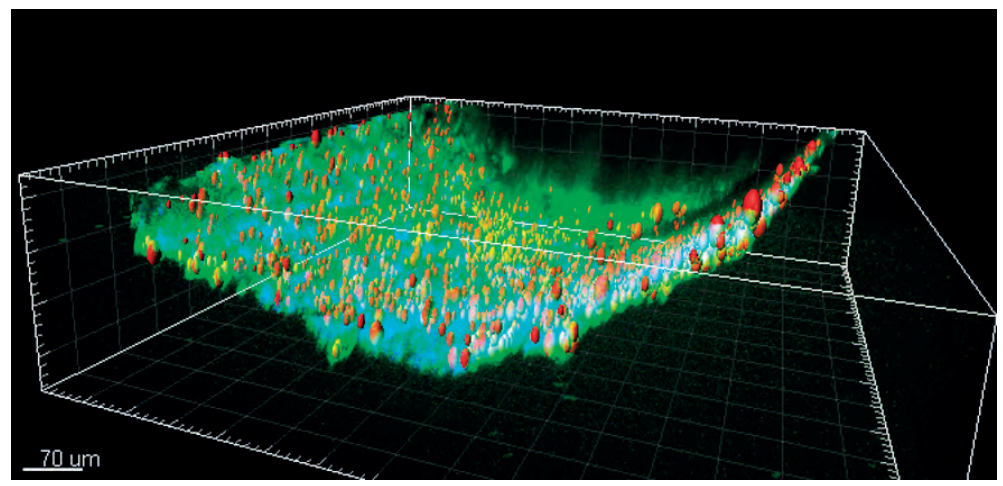

Figure 6.2 Using Imaris (C) software, image stacks of the two-photon laser scanning microscopy analysis were reconstructed in 3D. Here, a part of the reconstructed vascular wall (E2) is shown. Autofluorescent particles are reconstructed as well. They are represented by the reddish spherical particles and based on image threshold intensity. Particles are visualized in the combined (blue, green, red) channels and depicted as red spheres using the 'spots function', for a better visual discrimination against background signal. Collagen is visible in the blue channel due to second harmonic generation. Elastin is visible due to its autofluorescence in both the green channel (strong) and red channel (weak), resulting in a predominantly green signal. Finally, fluorescently labeled dextran proteins can be seen within the lumen as a green gauze.

\begin{tabular}{|c|c|c|c|c|}
\hline & E2 & E3 & E4 & $\mathrm{C} 1$ \\
\hline Total vessel wall volume included (micrometers ${ }^{3}$ ) * 10E6 & 94,39 & 2,13 & 0,64 & 8,70 \\
\hline Mean diameter per particle (micrometers) & 4.81 & 3.11 & 2.78 & 4.79 \\
\hline Mean volume per particle (micrometers ${ }^{3}$ ) & 163 & 44 & 30 & 145 \\
\hline $\begin{array}{l}\text { Density of particles in the vascular wall (number of } \\
\text { particles / total stack volume) } * 10 \text { E6 }\end{array}$ & 85 & 199 & 642 & 135 \\
\hline $\begin{array}{l}\text { Volume density of particles in the vascular wall } \\
\text { (percentage) [ volume particles (micrometers }{ }^{3} \text { ) / total } \\
\left.\text { stack volume (micrometers }{ }^{3}\right) * 100 \%\end{array}$ & $1.38 \%$ & $0.88 \%$ & $1.93 \%$ & $1.97 \%$ \\
\hline
\end{tabular}

Table 6.2 Quantification analysis of autofluorescent particles in pial arteries Properties of the autofluorescent particles within the pial artery of three patients and one control are presented in this table. In the upper row, the total vascular wall volume of included image stacks is shown. The number of particles, mean particle diameter and volume were included to calculate particle density and volume density of particles within the vascular wall. There is a high variation in particle size and number between patients and control but also between the patients. 

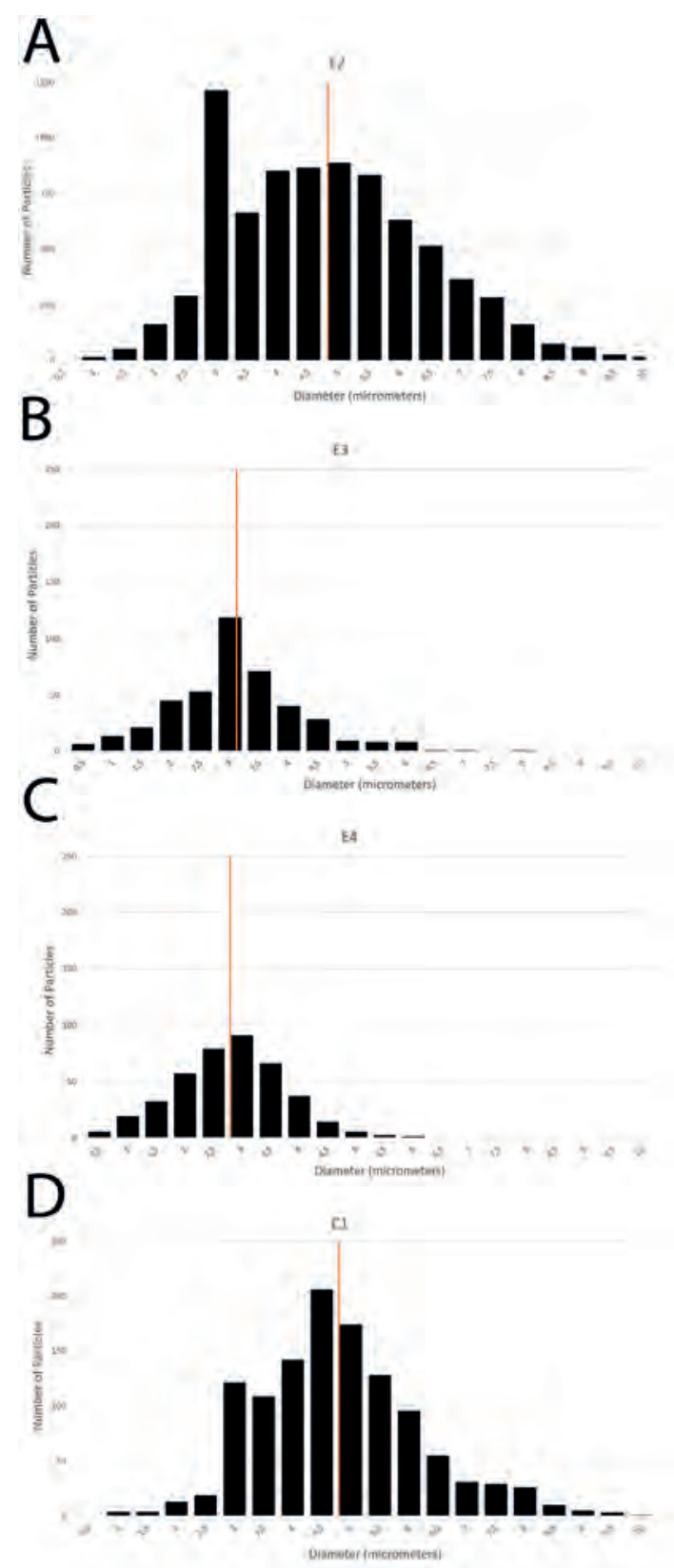

Figure 6.3 Particle diameters of the autofluorescent particles within the pial artery wall of three patients $(A, B, C)$ and one control (D). For each diameter, we have calculated the total number within the image stack, as obtained using two-photon laser scanning microscopy. Since the image stack of E2 (A) was evidently larger than the other image stacks, a 10-fold larger number of particles per diameter were noted in this patient. Most particles were found to have a diameter between 2 and 6 micrometers. The red vertical line shows the mean diameter. Mean diameter of E2 and C1 are higher than for E3 and E4. 


\subsection{Fluorescence spectroscopic imaging}

Autofluorescent particles (Nuance spectroscopy) on neocortical slides were predominantly found near or within parenchymal vessels, although numerous particles were noted throughout the tissue as well (Figure 6.4). At 100x magnification, these autofluorescent particles appeared to be mainly located in the adventitia of the parenchymal arterioles (Figure 6.5). This is consistent with our results on pial arteries using two-photon laser scanning microscopy (see above).
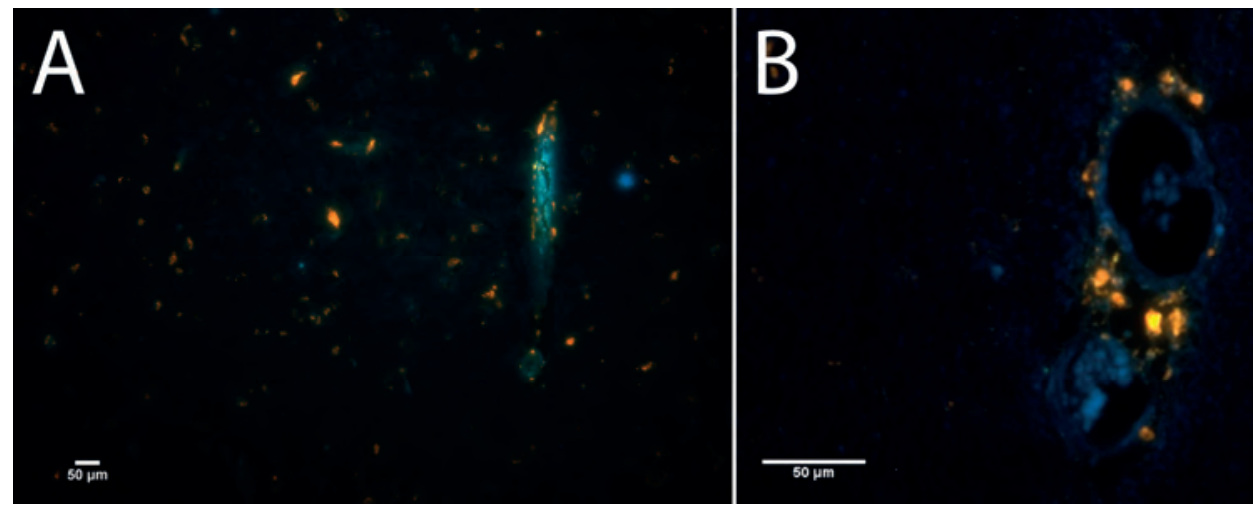

Figure 6.4 In A, a temporal neocortical tissue slice is shown. The autofluorescent particles are recognizable as yellow fluorescent particles. The particles are found throughout the tissue but are predominantly located around parenchymal vessels (B). These images are derived using Nuance microscopy at a 20x magnification.
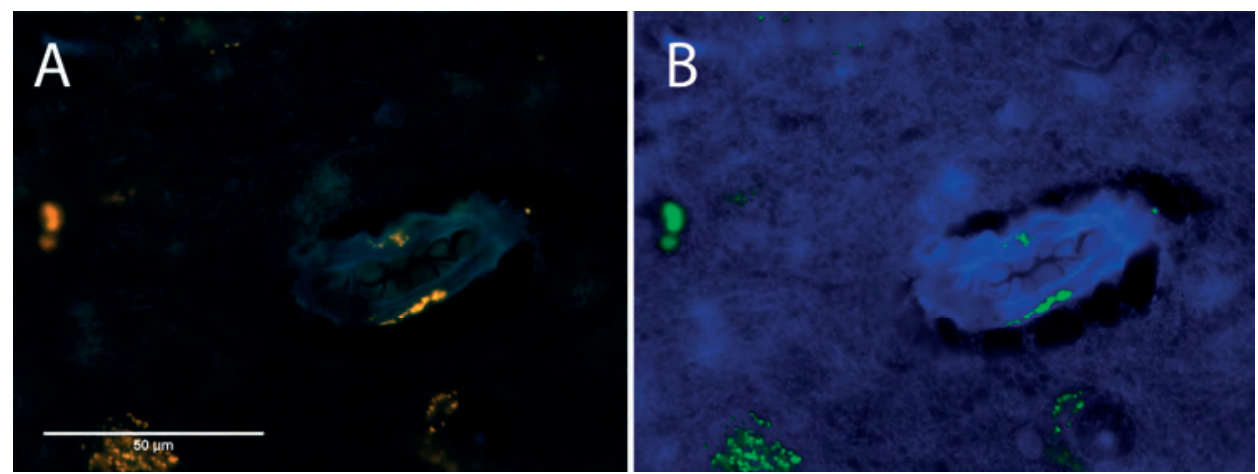

Figure 6.5 A) Autofluorescent yellowish particles within the wall of a parenchymal vessel. B) unmixed image. The lumen seems to contain a substance, probably a blood clot. Next, the endothelial layer is aligned by a brighter (pseudo-color) blueish layer, probably representing the internal elastic membrane. More outward in the vascular wall, the particles are noted. Hence, particles are most prominently located in the adventitia. As in figure 3, particles are also found within the neocortical tissue, where some seem to surround a small lumen as well, while no vascular lumen can be seen near other particles. These images are derived using Nuance microscopy at a 100x magnification. 
Independent of their localization, particles show a broad and sloping emission spectrum between 460 and $720 \mathrm{~nm}$ on excitation, with a maximum at 580 to $600 \mathrm{~nm}$ (Figure 6.6). Autofluorescence spectra were similar in both groups. The spectrum measured on the paraffin slides did not differ from that in fresh-frozen slides (not shown).
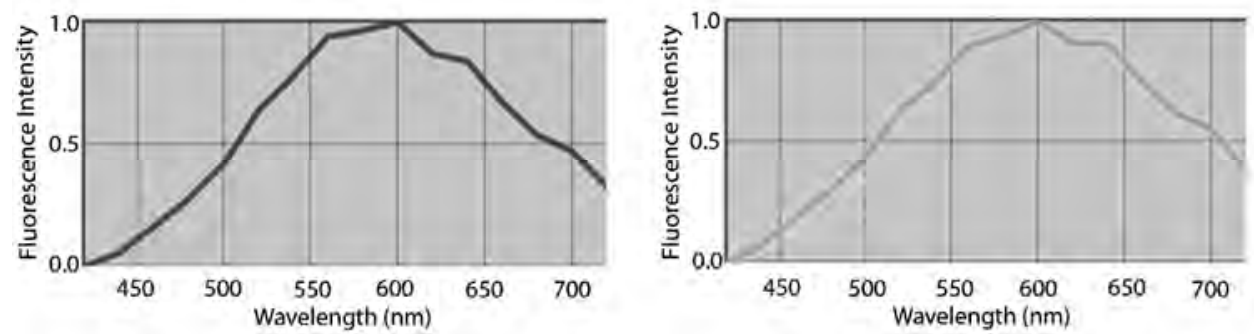

Figure 6.6 Emission spectrum of the autofluorescent aggregates within the neocortical tissue slides of a patient $(A)$ and a control $(B)$ is illustrated.

\subsection{Fluorescence lifetime imaging}

The fluorescence lifetime of autofluorescent particles surrounding parenchymal vessels was analyzed in one patient. First, spectrum analysis was repeated with two-photon laser scanning microscopy to verify that particles with similar spectral properties were analyzed. Subsequent autofluorescence intensity decay on pulsed excitation showed a biexponential decay. A fast component $\left(\tau_{1}\right)$ at $0.527+/-n s$ and a slow component $\left(\tau_{2}\right)$ at $2.60+/-$ ns were noted with a relative amplitude of $87+/-\%\left(\alpha_{1}\right)$ and $13+/-\%\left(\alpha_{2}\right)$, respectively. Based on these values, a mean lifetime of 1.41 t/- ns was calculated. Lifetime analysis of particles observed throughout the neocortical tissue showed similar results. A mean lifetime of $1.55+/-n s$ was calculated for those particles, based on $\tau_{1}$ at $0.530+/-n s$ and $\tau_{2}$ at $2.97+/-n s$, with a relative amplitude of $89+/-\%\left(\alpha_{1}\right)$ and $11+/-\%$ $\left(\alpha_{2}\right)$, respectively.

\subsection{Histochemistry}

As can be seen in Figure 6.7, fluorescent particles in the native slide could be matched with PAS-positive particles in the adjacent slide, $5 \mu \mathrm{m}$ apart (Figure 6.7). 


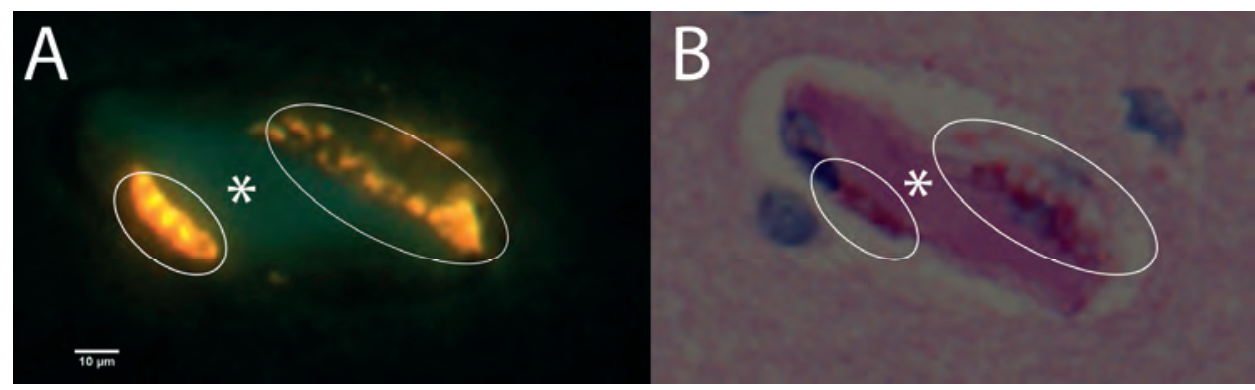

Figure 6.7 A) Autofluorescent (yellowish) particles (white ellipse) surrounding the lumen (white asterisk) of a parenchymal vessel can be noted when analyzed by Nuance microscopy at $40 x$ magnification. B) The 5 micrometers adjacent slide was stained using periodic acid Schiff's. Here, the autofluorescent particles are depicted as red-colored, PAS-positive, particles (white ellipse) surrounding the lumen (white asterisk) of the same parenchymal vessel.

\section{Discussion}

This is the first report demonstrating lipofuscin within the cerebral vascular wall. Identification of lipofuscin was based on autofluorescent properties and histological analysis. Considering lipofuscin as a marker for the total amount of oxidative stress endured, the accumulation of lipofuscin suggests oxidative stress in the cerebral vessel $^{4,5,27}$.

\subsection{Identification of lipofuscin}

In order to identify the autofluorescent particles, we performed a number of consecutive experiments, starting with the assessment of their autofluorescent properties. Fluorescence spectral analysis revealed a broad emission spectrum with a maximum between 580 to $600 \mathrm{~nm}$. These results are comparable to spectral properties of lipofuscin described in literature ${ }^{28-33}$. Since lipofuscin encompasses a wide variety of lipopigments, the exact autofluorescence spectrum is broad and depends on the ratio of varying fluorescent lipopigmental constituents ${ }^{29,34}$. Consequently, spectral variations between different tissues, and possibly between different species, have been described, in addition to variations due to distinct detection methods ${ }^{28,29,35}$. Nevertheless, autofluorescence analysis has been regarded as a reliable method to assess the presence of lipofuscin, both qualitatively and quantitatively ${ }^{29}$.

A similar broad autofluorescence spectrum is also found in advanced glycation end products $^{36}$. To distinguish between these and lipofuscin, fluorescence life time analysis was performed. We demonstrated a fluorescence lifetime of 1.41 to $1.55 \mathrm{~ns}$. We compared our measurements with the two other available studies on lipofuscin lifetime 
analysis. Both were carried out in retinal tissue ${ }^{32,33}$. The first study characterizes lipofuscin with a fast component $\left(\tau_{1}\right)$ at $0.390 \mathrm{~ns}$, a slow component $\left(\tau_{2}\right)$ at $2.24 \mathrm{~ns}$, and a mean lifetime of $1.98 \mathrm{~ns}$ based on associated relative amplitudes $\alpha_{1}$ of $48 \%$ and $\alpha_{2}$ at $52 \%^{32}$. In the same study, advanced glycation end products exhibit a bi-exponential decay with a fast component $\left(\tau_{1}\right)$ at $0.865 \mathrm{~ns}$ and a slow component $\left(\tau_{2}\right)$ at $4.17 \mathrm{~ns}$, with a relative amplitude of $62 \%\left(\alpha_{1}\right)$ and $28 \%\left(\alpha_{2}\right)$ respectively, resulting in a mean lifetime $\left(\tau_{\text {mean }}\right)$ of $3.13 \mathrm{~ns}^{32}$. In the other study, Berezin and Achilefu reported a mean lifetime of 1.34 ns for lipofuscin, but did not explain how this value was calculated $^{33}$. We calculated a $\tau_{\text {mean }}$ of 1.41 to $1.55 \mathrm{~ns}$, which is comparable to the other two reported values on lipofuscin lifetime.

Finally, we performed a PAS-staining, which revealed that the autofluorescent particles were PAS-positive on adjacent section slides. The PAS stain is not lipofuscin-specific, since it also stains glycogen and lipid droplets. However, the match of PAS-positivity to autofluorescent signal confirms that the particles are indeed lipofuscin, since neither glycogen nor lipid droplets hold autofluorescent properties.

\subsection{Quantification of particles}

Using Imaris (c) software, we have performed a quantitative analysis of the lipofuscin particles within the pial artery wall in three patients and one control. For this analysis, we have included the two-photon laser scanning microscopy Z-stack image files. Particle diameter, particle volume, and total vessel wall volume were estimated, and particle and volume density were subsequently calculated (Table 6.2).

Images of patient E2 included six Z-stack files, whereas all other image files included one Z-stack file. As a consequence, total vessel wall volume of E2 is much larger. Regarding mean particle diameter and volume, we noted that mean diameter of a particle is not proportionally related to its mean volume. This suggests that lipofuscin particles are not spherical but have a complex three-dimensional figuration. This is also visible from the volume reconstructions.

Since lipofuscin is thought to accumulate with age, an age-associated increase was assessed using control tissue ${ }^{37,38}$. Number of particles varied highly between patients, as well as between patients and the control. Nevertheless, density of lipofuscin particles was 1.5 to 4.8 times higher in two young (18 and 28 years old) patients compared to the control (61 years old). Comparing the size of the lipofuscin particles with the control, we found that these were smaller in patients E3 and E4, while of comparable size in patient E2.

Hence, pial arteries of young epilepsy patients are characterized by a comparable or higher number of equally or smaller sized lipofuscin particles when compared to the pial artery of an older control. One could hypothesize that smaller particles are related to brief periods of oxidative stress, whereas larger particles are the results of sustained oxidative stress. During life, cells are exposed to both durations of oxidative stress leading to the formation of smaller and larger lipofuscin particles. During seizures, 
epilepsy patients are frequently exposed to brief periods of oxidative stress. This might result in an increased number of smaller sized particles.

These results suggest an epilepsy-associated lipofuscin increase rather than an ageassociated increase. However, for a thorough understanding of an age-associated lipofuscin increase we need an extensive biobank containing brain and cerebral vessel samples of otherwise healthy individuals of all ages. In the much regretted absence of such data, and looking for a young control subject, we examined middle cerebral artery samples of a 25-weeks old Wistar-Kyoto rat, corresponding with approximately 18 human years ${ }^{39}$. Hardly any autofluorescent particles were observed within the vessel wall in these samples. However, it should be noted that a rat's middle cerebral artery is probably not completely comparable to a human pial artery. If otherwise healthy humans have comparable low levels of vascular lipofuscin, the high lipofuscin levels in our patients suggest an epilepsy-associated formation of lipofuscin that is independent of age. Therefore, we interpret this suggestion in the light of known vascular abnormalities in epilepsy patients.

Pial arteries are responsible for cerebrovascular autoregulation, rendering cerebral blood flow independent of changes in arterial pressure within a certain range. During seizures, blood pressure and heart rate rise acutely to sometimes peak values ${ }^{40}$. Consequently, autoregulatory mechanisms of pial arteries are repetitively challenged in patients suffering from chronic seizures, possibly leading to oxidative stress within pial vascular cells ${ }^{41}$.

Pial arteries subsequently branch into penetrating parenchymal arterioles involved in neurovascular coupling, the mechanism regulating local blood flow based on metabolic demands of the cerebral parenchyma. This coupling mechanism originates in neuronal and vascular cell signalling at the capillary level. When metabolic demands increase, it induces vasodilatation of upstream parenchymal arterioles to increase local blood flow $^{17}$. In epilepsy, seizures induce acutely elevated metabolic demands, frequently challenging the neurovascular coupling mechanisms and vascular responses of parenchymal arterioles to accommodate the acutely changing and highly variable blood flow $^{42}$.

Physiologic neuronal and vascular cell signalling at the capillary level is often disturbed in epilepsy patients. This is mainly due to an increased permeability of the blood-brain barrier inducing disturbed homeostasis, local inflammation, neuronal dysfunction, and hypoxia ${ }^{18,21}$. As a consequence, neurovascular coupling might be disrupted leading to a dysfunctional upstream neurovascular response, while this response is simultaneously challenged by the extreme metabolic demands. Such a non-synchronous and inadequate coupling mechanism has indeed been described in epilepsy patients ${ }^{43}$. Similar pathophysiologic neurovascular mechanisms have also been reported in other neurodegenerative diseases, such as Alzheimer's disease, in which oxidative stress plays a major pathophysiologic role ${ }^{17}$. 
In epilepsy patients, mitochondrial dysfunction of neuronal cells has been demonstrated and is linked to seizure susceptibility and epileptogenesis ${ }^{24,44-47}$. Since mitochondrial dysfunction leads to increased oxidative stress, its presence in (neuro)vascular cells of epilepsy patients may also contribute to lipofuscin formation by oxidative stress in these cells ${ }^{4,5}$.

In our study, we also noted lipofuscin particles throughout the neocortical tissue of young patients and older controls. However, we did not further assess the exact cellular localization of these particles, e.g. within vascular cells of small arterioles or capillaries or within parenchymal cells. When these particles would be located within capillary vascular and nearby neuronal and glial cells, this might further emphasize the presence of oxidative stress in these cells perhaps reflecting vascular dysfunction in epilepsy. Oxidative stress within parenchymal cells of epilepsy patients has been reported on earlier and might either further contribute to vascular dysfunction or be a consequence of vascular dysfunction ${ }^{46}$.

Summarized, cerebral vascular cells are repetitively exposed to oxidative stress due to the extreme and rapidly changing demands, dysfunctional neurovascular mechanisms, and structural abnormalities. Not surprisingly, both aberrant parenchymal vessels and increased angiogenesis, reflecting vascular dysfunction and insufficient blood supply, have been reported in epilepsy patients ${ }^{20,48}$.

\subsection{Importance of this study}

The demonstration of lipofuscin, based on spectrometric characteristics, could be a new method to assess the presence of oxidative stress. Using additional software, particle size and density characteristics can easily be assessed. Currently, the assessment and quantification of oxidative stress is challenging. Since reactive oxygen species are transient and highly unstable, indirect markers for oxidative stress are mainly used ${ }^{46}$. Indirect markers include lipid and protein oxidation and the activity of free radical scavenging enzymes ${ }^{46,49}$. Assays to measure these surrogates often lack sensitivity and specificity for the process they are supposed to depict ${ }^{24}$. In contrast, we have shown lipofuscin is easily visualized using adequate microscopy techniques and additional software allows straightforward and reliable quantification. Lipofuscin is a stable marker due to its non-degradable nature. However, formation of lipofuscin is the result of slowly accumulative oxidative stress limiting the oxidative stress effect of a single and/or brief oxidative challenge. Hence, lipofuscin can provide insight in the long-term amount of endured oxidative stress during the course of (neurodegenerative) diseases.

The finding of lipofuscin within vascular cells suggests oxidative stress within the vascular wall. Whether oxidative stress within the cerebral vascular wall is a physiological finding is unknown. In this study, we have mainly included young epilepsy patients and also found a remarkably high number of lipofuscin in some of these patients in comparison to an older control. This suggests that vascular cells in pial 
arteries and parenchymal vessels of these patients are exposed to increased oxidative stress. This may be the result of structural and functional vascular abnormalities in extreme and rapidly changing hemodynamic conditions related to the underlying disease, i.e. epilepsy.

\section{Conclusion}

We have demonstrated the presence of lipofuscin in the cerebral vascular wall based on its autofluorescent properties. Analysis of lipofuscin could be a new method to detect and quantify oxidative stress.

Future studies on lipofuscin in epilepsy patients and healthy, variably aged controls contribute to a better understanding of vascular wall function and dysfunction in relation to oxidative stress, and vascular dysfunction as is seen in epilepsy. 


\section{References}

1. Czerska M, Mikołajewska K, Zieliński M, Gromadzińska J, Wąsowicz W. Today's oxidative stress markers. Med Pr. 2015;66:393-405.

2. Lushchak VI. Free radicals, reactive oxygen species, oxidative stress and its classification. Chem Biol Interact. 2014;224:164-75.

3. Sies H. Oxidative stress: oxidants and antioxidants. Exp Physiol. 1997;82:291-5.

4. Brunk UT, Terman A. Lipofuscin: mechanisms of age-related accumulation and influence on cell function. Free Radic Biol Med. 2002;33:611-9.

5. Brunk UT, Terman A. The mitochondrial-lysosomal axis theory of aging: Accumulation of damaged mitochondria as a result of imperfect autophagocytosis. Eur J Biochem. 2002;269:1996-2002.

6. Terman A, Brunk UT. Lipofuscin: mechanisms of formation and increase with age. APMIS. 1998;106:265-76.

7. Terman A, Brunk UT. Lipofuscin. Int. J. Biochem. Cell Biol. 2004;36:1400-4.

8. Jung T, Höhn A, Grune T. Lipofuscin: Detection and quantification by microscopic techniques. Methods Mol Biol. 2010;594:173-93.

9. Tohma H, Hepworth AR, Shavlakadze T, Grounds MD, Arthur PG. Quantification of ceroid and lipofuscin in skeletal muscle. J. Histochem. Cytochem. 2011;59:769-79.

10. Strehler BL, Mark DD, Mildvan AS, Gee M V. Rate and magnitude of age pigment accumulation In the human myocardium. J Gerontol. 1959;14:430-9.

11. Najjar S, Pearlman DM, Devinsky O, Najjar A, Zagzag D. Neurovascular unit dysfunction with blood-brain barrier hyperpermeability contributes to major depressive disorder: a review of clinical and experimental evidence. J Neuroinflammation. 2013;10:142.

12. Ng F, Berk M, Dean O, Bush Al. Oxidative stress in psychiatric disorders: evidence base and therapeutic implications. Int J Neuropsychopharmacol. 2008;11:851-76.

13. Scapagnini G, Davinelli S, Drago F, De Lorenzo A, Oriani G. Antioxidants as antidepressants: Fact or fiction? CNS Drugs. 2012;26:477-90.

14. Islam MT. Oxidative stress and mitochondrial dysfunction-linked neurodegenerative disorders. Neurol Res. Taylor \& Francis; 2017;39:73-82.

15. Brown WR, Thore CR. Review: Cerebral microvascular pathology in aging and neurodegeneration. Neuropathol Appl Neurobiol. 2011;37:56-74.

16. Heinemann U, Kaufer D, Friedman A. Blood Brain Barrier Dysfunction, TGFb signaling and AStrocyre Dysfunction in Epilepsy. Glia. 2012;60:1251-7.

17. Iadecola C. Neurovascular regulation in the normal brain and in Alzheimer's disease. Nat Rev Neurosci. 2004;5:347-60.

18. Marchi N, Lerner-Natoli M. Cerebrovascular remodeling and epilepsy. Neurosci. 2013;19:304-12.

19. Parfenova H, Carratu P, Tcheranova D, Fedinec A, Pourcyrous M, Leffler CW. Epileptic seizures cause extended postictal cerebral vascular dysfunction that is prevented by HO-1 overexpression. AJP Hear Circ Physiol. 2005;288:H2843-50.

20. Rigau V, Morin M, Rousset M-C, et al. Angiogenesis is associated with blood-brain barrier permeability in temporal lobe epilepsy. Brain. 2007;130:1942-56.

21. van Vliet EA, Aronica E, Gorter JA. Blood-brain barrier dysfunction, seizures and epilepsy. Semin Cell Dev Biol. 2015;38:26-34.

22. Marchi N, Betto G, Fazio V, et al. Blood-brain barrier damage and brain penetration of antiepileptic drugs: Role of serum proteins and brain edema. Epilepsia. 2009;50:664-77.

23. Rowley S, Patel M. Mitochondrial Involvement and Oxidative Stress in Temporal Lobe Epilepsy. Free Radic Biol Med. 2013;62:121-31.

24. Waldbaum S, Patel M. Mitochondria, oxidative stress, and temporal lobe epilepsy. Epilepsy Res. 2010;88:23-45.

25. Zsurka G, Kunz WS. Mitochondrial dysfunction and seizures: the neuronal energy crisis. Lancet Neurol. 2015;14:956-66.

26. Sillen A, Engelborghs Y. The Correct Use of "Average" Fluorescence Parameters. Photochem. Photobiol. 1998;67:475-86. 
27. Gray DA, Woulfe J. Lipofuscin and aging: a matter of toxic waste. Sci Aging Knowledge Environ. 2005; $1--5$.

28. Seehafer SS, Pearce DA. You say lipofuscin, we say ceroid: Defining autofluorescent storage material. Neurobiol Aging. 2006;27:57688.

29. Mochizuki Y, Park MK, Mori T, Kawashima S. The difference in autofluorescence features of lipofuscin between brain and adrenal. Zoolog Sci. 1995:283-8.

30. Marmorstein AD, Marmorstein LY, Sakaguchi H, Hollyfield JG. Spectral profiling of autofluorescence associated with lipofuscin, Bruch's membrane, and sub-RPE deposits in normal and AMD eyes. Investig. Ophthalmol Vis Sci. 2002;43:2435-41.

31. Sparrow JR, Boulton M. RPE lipofuscin and its role in retinal pathobiology. Exp Eye Res. 2005;80: 595-606.

32. Schweitzer D, Schenke S, Hammer M, et al. Towards metabolic mapping of the human retina. Microsc Res Tech. 2007;70:410-9.

33. Berezin MY, Achilefu S. Fluorescence lifetime measurements and biological imaging. Chem Rev. 2011;110:2641-84.

34. Katz ML, Robison WG. What is lipofuscin? Defining characteristics and differentiation from other autofluorescent lysosomal storage bodies. Arch Gerontol Geriatr. 2002;34:169-84.

35. Yin DZ, Brunk UT. Microfluorometric and fluorometric lipofuscin spectral discrepancies: a concentration-dependent metachromatic effect? Mech Ageing Dev. 1991;59:95-109.

36. Marcu L, French PMW, Elson DS. Fluorescence lifetime spectroscopy and imaging. Boca Raton: CRC Press, Taylor \& Francis Group; 2015.

37. Goyal VK. Lipofuscin pigment acculumaltion in human brain during ageing. Exp. Gerontol. 1983;17: 481-7.

38. Benavides SH, Monserrat AJ, Fariña S, Porta EA. Sequential histochemical studies of neuronal lipofuscin in human cerebral cortex from the first to the ninth decade of life. Arch Gerontol Geriatr. 2002;34: 219-31.

39. Sengupta P. The laboratory rat: Relating its age with human's. Int. J. Prev. Med. 2013:624-30.

40. Iadecola C, Davisson RL. Hypertension and cerebrovascular dysfunction. Cell Metab. 2008;7(6):476-84.

41. Devinsky O. Effects of Seizures on Autonomic and Cardiovascular Function. Epilepsy Curr. 2004;4:43-6.

42. Zhao M, Nguyen J, Ma H, Nishimura N, Schaffer CB, Schwartz TH. Preictal and ictal neurovascular and metabolic coupling surrounding a seizure focus. J Neurosci. 2011;31:13292-300.

43. Ma H, Zhao M, Schwartz TH. Dynamic neurovascular coupling and uncoupling during ictal onset, propagation, and termination revealed by simultaneous in vivo optical imaging of neural activity and local blood volume. Cereb Cortex. 2013;23(4):885-99.

44. Folbergrová J, Kunz WS. Mitochondrial dysfunction in epilepsy. Mitochondrion. Elsevier B.V. and Mitochondria Research Society; 2012;12:35-40.

45. Kudin AP, Zsurka G, Elger CE, Kunz WS. Mitochondrial involvement in temporal lobe epilepsy. Exp Neurol. 2009;218:326-32.

46. Patel M. Mitochondrial dysfunction and oxidative stress: cause and consequence of epileptic seizures. Free Radic Biol Med. 2004;37:1951-62.

47. Rahman S. Pathophysiology of mitochondrial disease causing epilepsy and status epilepticus. Epilepsy Behav. 2015;49:71-5.

48. Alonso-Nanclares L, DeFelipe J. Alterations of the microvascular network in the sclerotic hippocampus of patients with temporal lobe epilepsy. Epilepsy Behav. 2014;38:48-52.

49. Bruce AJ, Baudry M. Oxygen free radicals in rat limbic structures after kainate-induced seizures. Free Radic Biol Med. 1995;18:993-1002. 



\section{Chapter \\ 7}

General discussion and conclusions 



\section{General discussion and conclusions}

In human epilepsy and more specifically in temporal lobe epilepsy (TLE), structural and functional microvascular abnormalities have been described in literature. The aim of this thesis was to explore various structural and functional cerebrovascular topics and their role in relation to the pathophysiology of TLE. To this end, the first topic of this thesis focused on the cerebrovascular glycocalyx that could be an important structure of the cerebral microcirculation. In chapter $\mathbf{2}$, several techniques that allow glycocalyx assessment are described. Based on this literature study, we selected sidestream dark field (SDF)-imaging to analyze the human cerebrovascular glycocalyx during resective brain surgery. Next, we designed a study to utilize this technique during surgical treatment of patients suffering from drug-resistant TLE, and of control patients without epilepsy. The study design was presented in chapter $\mathbf{3}$ and was followed by a feasibility study described in chapter 4.

The next topic of this thesis addressed vasomotor control of cerebral arteries. Analysis of this arterial function was particularly focused on neurogenic, adrenergic and Endothelin-1 mediated vasoconstriction of cerebral arteries (chapter 5). Finally, chapter 6 was dedicated to the qualitative and quantitative analysis of autofluorescent particles that were observed within the vascular wall of pial arteries from drug-resistant epilepsy patients.

In this general discussion, the three topics of this thesis will be discussed more thoroughly. This discussion follows the order of hypotheses and research questions delineated in the general introduction of this thesis.

\section{Hypothesis I}

\section{The cerebrovascular glycocalyx is disrupted in temporal lobe epilepsy patients}

The glycocalyx is a gel-like matrix lining the luminal side of endothelial cells ${ }^{1}$. Attached to the endothelium, the glycocalyx is involved in important endothelial functions ${ }^{2-4}$. First of all, the glycocalyx forms a barrier between blood cells and components and endothelial cells, which is exemplified by the prevention of albumin extravasation due to the electrically negatively charged sulfated glycosaminoglycan side chains within the glycocalyx ${ }^{5}$. The glycocalyx also regulates vascular inflammatory responses by modulating leucocyte trafficking ${ }^{6,7}$. Moreover, endothelial remodeling and activation of endothelial vasoactive substances in response to shear stress are the result of mechanotransduction by the glycocalyx ${ }^{4,8}$. Hence, a disturbed glycocalyx could contribute to and/or be the result of endothelial dysfunction. In the cerebral capillary bed, endothelial cells are considered key players in determining permeability of the socalled blood-brain barrier (BBB) in order to maintain cerebral homeostasis ${ }^{9}$. 
In epilepsy, structural endothelial abnormalities within the cerebral microcirculation have been described such as a reduced number of tight junctions and a decreased endothelial - basal lamina connectivity ${ }^{10-12}$. Functionally, increased BBB permeability has been demonstrated in TLE-patients repetitively ${ }^{10,11,13}$. It has been found that this increased permeability results in albumin leakage, that in turn orchestrates astrocyte transformation by activating transforming growth factor $\beta$ signalling ${ }^{11,14}$. This transformation is characterized by downregulation of inward rectifier potassium channels and astrocytic glutamate transporters, resulting in enhanced neuronal excitability, upregulation of proinflammatory cytokines, altered neurovascular coupling, and impaired brain homeostasis ${ }^{14,15}$. Upregulation of proinflammatory cytokines as a response to trafficking of leucocytes further contributes to increased neuronal excitability $^{15,16}$

In summary, endothelial structural and functional alterations may contribute to the pathophysiology of epilepsy. Whether a disrupted glycocalyx can be a cause or a consequence of this pathophysiological mechanism is currently unknown. A healthy glycocalyx is known to prevent extravasation of large proteins like albumin and inhibit leukocyte trafficking in capillary beds throughout the body ${ }^{17,18}$. Nevertheless, the existence and properties of the cerebrovascular glycocalyx have only sparsely been reported on ${ }^{19,20}$. Not surprisingly, current structural and functional concepts of cerebral capillaries rarely include the endothelial glycocalyx.

A recent preclinical study has demonstrated that degradation of the cerebrovascular glycocalyx increased BBB permeability and subsequently induced cerebral edema, local neuroinflammation and neuronal cell loss ${ }^{21}$. Noteworthy, this study reported that all rats in whom the glycocalyx was disrupted suffered from status epilepticus. These findings suggest that the glycocalyx is a significant structural and functional component of the cerebral capillary endothelium and that disruption of the glycocalyx might induce seizures. This is in line with our hypothesis that a disrupted glycocalyx may contribute to the pathophysiology of temporal lobe epilepsy.

To address this hypothesis, we first aimed to analyze the currently available state-ofthe-art techniques that are used to assess the cerebrovascular glycocalyx. This brings us to our first research question:

\section{What are the available techniques to assess the cerebrovascular glycocalyx?}

In chapter 2, a literature study is presented evaluating seven techniques that have been used to assess the cerebrovascular glycocalyx. It was found that study conditions, like in vitro versus in vivo or preclinical versus clinical, determine which technique is best applied. In this thesis, we aimed to assess the cerebrovascular glycocalyx in TLEpatients during resective surgery, i.e. in vivo. Based on the literature study, we concluded that side-stream dark field (SDF) imaging is the most suitable technique to assess the human cerebrovascular glycocalyx in vivo. This technique allows in vivo visualization of the microcirculation. By adding specific software, SDF-imaging enables real-time, non-invasive, quantitative evaluation of the microvascular glycocalyx without 
the use of fluorescent dyes. However, this technique does not allow qualitative evaluation of the glycocalyx, i.e. analysis of its components, and is prone to motion and pressure artefacts.

SDF-imaging evaluates glycocalyx health based on the perfused boundary region (PBR). The PBR represents the more dynamic and permeable part of the glycocalyx located most luminally. An increased PBR suggests a more permeable and unstable glycocalyx indicating a potentially reduced glycocalyx integrity, whereas a low PBR indicates a well-preserved glycocalyx. The PBR is calculated based on variations in the width of the red blood cell column within cerebral vessels with a diameter of 5-20 $\mu \mathrm{m}$. SDF-imaging technique is based on the illumination of (de-)oxyhemoglobin within red blood cells, thereby enabling the evaluation of the red blood cell column width variation and hence the calculation of the PBR. The second research question of this thesis aimed to apply our theoretical finding that SDF-imaging enables intraoperative analysis of the cerebrovascular glycocalyx:

\section{Can the cerebrovascular glycocalyx be assessed using side-stream dark field imaging during resective brain surgery?}

In the light of hypothesis I, an explorative clinical case-control study was designed to assess the cerebral microcirculation and glycocalyx in TLE-patients and control patients without epilepsy using SDF-imaging (chapter 3). Next, it was attempted to visualize the cerebral microcirculation and additionally assess its glycocalyx intraoperatively. The results of the first measurements are described in a technical feasibility study of this technique (chapter 4). This study confirmed that SDF-imaging is a suitable technique for in vivo assessment of the human cerebrovascular glycocalyx. To the best of our knowledge, this is the first report on the in vivo assessment of the human cerebrovascular glycocalyx.

In chapter 4, the preliminary results of the measurements performed in TLE-patients are presented. A comparison of the preliminary results of TLE-patients and controls was not substantiated statistically and was not within the scope of this chapter: to show technical feasibility.

These preliminary results showed that the PBR of cortical microvessels was substantially larger (i.e. indicating a less stable and more permeable glycocalyx) than that of sublingual and hippocampal microvessels. This observation from cortical measurements may support the suggestion that the microcirculation of TLE patients is characterized by a disrupted glycocalyx. However, the glycocalyx of the hippocampal microcirculation of these patients was less permeable than that of the cortical microcirculation. This is remarkable as many reports on microvascular dysfunction in TLE-patients included hippocampal tissue, and hippocampal sclerosis is a common histopathological finding in these patients ${ }^{11,12,22}$. Hence, results on the cortical and hippocampal microvascular PBR will be correlated to histopathological findings of the temporal neocortex and hippocampus upon completion of this study. These preliminary 
data reflect measurements from only five TLE-patients and controls were not included. This warrants a cautious interpretation of these results.

\section{Microvascular density}

When performing SDF-imaging on the cerebral microcirculation, other microvascular properties such as vessel density, red blood cell velocity, and blood flow are also assessed. To date, assessment of the cerebral microcirculation in epilepsy patients has only been performed ex vivo by histologic evaluation ${ }^{12,23-25}$. This assessment does however not allow a functional hemodynamic analysis of the microcirculation. In vivo assessment of the cerebral microcirculation is no novelty. There are a few studies that evaluated the cerebral microcirculation using SDF-imaging or similar techniques ${ }^{26-29}$. However, none of these studies performed this assessment in relation to neurological disorders, like epilepsy. Chapter $\mathbf{4}$ describes microvascular density as a proof-ofprinciple that other microvascular properties could be assessed using this experimental setup. None of the delineated research questions was aimed at microvascular density in epilepsy. Nevertheless, the results described in chapter 4 will be elaborated on below as they are of interest when regarding microvascular abnormalities in epilepsy patients, which is the underlying topic of this thesis.

In epilepsy patients, increased and decreased microvascular densities have previously been described ${ }^{12,30-32}$. One could hypothesize that rapid and extreme increases in metabolic demands during seizures may result in an increased vessel density ${ }^{33}$. This is the result of metabolic stress during these seizures. The longer this metabolic stress lasts, the more deleterious its effects will be on cerebral tissue ${ }^{34}$. Consequently, cerebral blood flow will be adjusted to meet metabolic demands by neurovascular coupling ${ }^{35}$. However, an increased cerebral blood flow might not be sufficient as this will only increase red blood velocity, has no effect on the total vessel surface available for delivery and exchange of vital nutrients and does not alter the distance of a tissue cell to capillaries. An additional increase in vessel density, i.e. recruitment of capillaries, would therefore be desirable. This would increase total vessel surface available for delivery and exchange of vital nutrients across the BBB. At the same time, the distance of neuronal cells to nearby capillaries will be decreased, reducing the time required for nutrients to reach these cells. This could limit hypoxia and cell death caused by the metabolic challenges during the repetitive seizures in drug-resistant epilepsy patients ${ }^{36}$. Indeed, angiogenesis and increased microvascular density in the hippocampus of TLEpatients have been described ${ }^{12,24,25}$. All in all, this compensatory response would increase vessel density and local blood flow to meet extreme metabolic demands during seizures.

On the contrary, vascular dysfunction as described above might cause an insufficient compensatory capillary recruitment leading to a failure of the local microcirculation to meet seizure-related extreme metabolic demands. This would result in hypoxia 
accompanied by edema and inflammation inducing neuronal cell death ${ }^{37}$. Consequently, downstream cerebral tissue would be characterized by a decreased vessel density, gliosis, and neuronal cell loss. A common histopathological finding in the hippocampus of TLE-patients is hippocampal sclerosis which is characterized by neuronal cell loss and gliosis ${ }^{22}$. It is tempting to suggest that failure of capillary recruitment in response to metabolic alterations results in neuronal cell loss and gliosis. Hence, one would expect a decrease in vessel density in hippocampal sclerosis which has also been proposed by Alonso-Nanclares et al. ${ }^{32}$. Future results of our case-control study will include the correlation of microvascular properties to hippocampal and cortical histopathological findings to assess whether histopathological hallmarks of epilepsy are related to increased or decreased microvascular density.

In chapter 4, we reported that vessel density of the neocortical microcirculation appears lower than sublingual vessel density. The cerebral measurements were performed with a sterile slipcover, perhaps influencing vessel recognition. Nevertheless, the temporal neocortical vessel density was also reduced when comparing it to the hippocampal vessel density which was also performed using this slipcover. These findings might represent a physiological difference between temporal neocortex and hippocampus. Alternatively, they reflect failure of the neocortical microcirculation to adjust to increased metabolic demands or signify microvascular recruitment in the hippocampal. Future results upon completion of the study will allow more firm conclusions on the observed differences in microvascular properties of TLE patients and controls, and between measurement sites.

\section{Hypothesis II}

Cerebral artery vasoconstriction is controlled by endothelin-1 and is altered in epilepsy patients

\section{Does endothelin-1 play a role in cerebral artery vasoconstriction?}

Endothelin-1 (ET-1) is generally considered to be one of the most potent and longlasting vasoconstrictors. It has been implicated in various disorders including hypertension and vasospasm following subarachnoid hemorrhage ${ }^{38,39}$. It is however not known if ET-1 mediated vasoconstriction is important for physiological vasoconstriction or if it should only be considered in pathophysiologic processes. Pagán et al. recently described that electrical field stimulation (EFS)-induced vasoconstriction of healthy internal mammary arteries was reduced in the presence of an ET-1 antagonist, thereby suggesting a physiological role for $\mathrm{ET}-1^{40}$.

Classically, sympathetic adrenergic perivascular innervation is held responsible for most of the vasoconstrictor tone ${ }^{40,41-43}$. In cerebral arteries, however, contradictive findings 
have been reported regarding vasoconstrictive responses upon sympathetic nerve stimulation $^{44-48}$. In chapter $\mathbf{5}$, sympathetic nerve stimulation-induced vasoconstriction of human pial arteries from drug-resistant epilepsy patients was assessed using EFS. Application of EFS only elicited vasoconstriction in the presence of exogenously applied noradrenalin (NA). Moreover, this synergistic neurogenic and adrenergic-induced vasoconstriction appeared ET-1 dependent. This observation led to the hypothesis that NA stimulates the release of ET-1 from endothelial cells and/or perivascular sensory nerves. Upon release, ET-1 binds to the $\mathrm{ET}_{\mathrm{A}}$-receptor that is expressed by vascular smooth muscle cells to induce cerebral artery vasoconstriction (see figure 5.6, chapter 5). These results are in line with the results by Pagán et al., and emphasize the important role of ET-1 in cerebral artery vasoconstriction as suggested earlier ${ }^{40,49}$.

The main function of pial arteries is autoregulation of cerebral blood flow. Autoregulation is the mechanism that regulates cerebral blood flow within a range by changing vascular tone independent of changes in arterial pressure ${ }^{50}$. During seizures, blood pressure and heart rate can quickly rise to extreme levels, thereby challenging the autoregulatory responses of cerebral arteries ${ }^{51}$. These autoregulatory responses are repetitively challenged in patients suffering from chronic drug-resistant epilepsy. In addition, the sudden and repetitive increases in tissue metabolism that accompany seizures may further challenge the regulation of downstream cerebral blood flow, i.e. in parenchymal arterioles ${ }^{33}$.

Blood flow control in pial arteries depends on autoregulatory, metabolic, neurogenic and endothelial stimuli ${ }^{52-56}$. Dysfunction of one or more of these vasomotor regulating mechanisms may ultimately disrupt blood flow control. To assess whether vasomotor function of pial artery vasoconstriction is abnormal in epilepsy patients, our results need to be compared to non-epileptic controls. For obvious reasons, freshly obtained human brain tissue from healthy controls is not available. Therefore, cerebral arteries of healthy rats were used as controls.

Compared to pial arteries from epilepsy patients, rat middle cerebral arteries were more sensitive to cumulative concentrations of ET-1 and serotonin. However, both artery types were lacking a response to cumulative concentrations of NA. In contrast, pial arteries from epilepsy patients showed a more pronounced contractile response to the simultaneous application of EFS and NA than rat middle cerebral arteries. Subsequent responses to the addition of tetrodotoxin and an $\mathrm{ET}_{\mathrm{A}}$-receptor antagonist were comparable in both types of arteries. In contrast, guanethidine partially reversed the EFS / NA induced vasoconstriction only in pial arteries from epilepsy patients.

The observed differences between rat middle cerebral arteries and pial arteries from epilepsy patients can be caused by various factors. For instance, one cannot reliably compare arteries of two different species within a disease - control model. Reported arterial vasoconstrictive and vasodilatory responses to vasoactive substances may vary between rat and human arteries ${ }^{57,58}$. Moreover, middle cerebral arteries are first order cerebral arteries, whereas pial arteries are final order arteries. Reported differences 
between these types of cerebral arteries include variation in adventitial nerve- and receptor type, density, distribution, and sensitivity ${ }^{59-61}$. In addition, the samples from the epilepsy patients were obtained during surgery. A possible effect of anesthesia should hence be taken into account, since anesthesia is known to affect vasomotor responses ${ }^{62-64}$. On the other hand, rat middle cerebral artery samples were obtained post mortem, and rats were euthanized using $\mathrm{CO} 2$, which might also affect these responses. Finally, epilepsy-related factors may have contributed to the observed differences. For example, several anti-epileptic drugs have been reported to affect vasoreactivity and vascular structure ${ }^{65-67}$.

Nevertheless, one could also consider the described differences as epilepsy-specific. As such, we observed a more pronounced vasoconstriction in response to the synergistic stimulation of EFS and NA in pial arteries from epilepsy patients. One could hypothesize that this is an excessive vasoconstrictive response. Excessive vasoconstriction of pial arteries may result in an unintended reduced cerebral blood flow in the downstream capillary bed leading to a disturbed parenchymal homeostasis in these patients. In chronic focal epilepsy, altered autoregulatory and coupling mechanisms have indeed been described. Regarding autoregulation, Zhao et al. described a pre-ictal vasoconstriction of pial arteries feeding perifocal tissue, whereas a late ictal increase of cerebral blood flow was noted within in the epileptic focus ${ }^{33}$. These pre-ictal hemodynamic alterations are most likely anticipating the ictal event. A perturbed neurovascular coupling mechanism has been reported in chronic epilepsy patients. Seizures result in an increase in local cerebral blood flow ${ }^{68}$. However, this increase evolved much slower and lasted for a longer period than metabolic stress during these seizures in these epilepsy patients. This suggests a non-synchronous coupling mechanism ${ }^{69}$. The underlying mechanisms of this non-synchronous coupling has not been studied to date. These findings therefore warrant further studies on the hypothesis that a disturbed autoregulation and neurovascular coupling mechanisms could form a primary substrate facilitating seizures.

Further experiments are required to elucidate the physiological mechanisms by which NA modulates ET-1 and to assess the role of other co-transmitters. In order to address alterations of cerebral artery vasomotor control in epilepsy, a preclinical epilepsy model may reveal differences between cerebral artery vasoconstriction under physiological and epilepsy-related conditions. 


\section{Hypothesis III}

The vascular wall of cerebral arteries of epilepsy patients shows signs of oxidative stress

\section{What is the nature of the autofluorescent particles noted in the vascular wall of epilepsy patients?}

Chapter 6 describes a series of experiments that aimed to visualize and characterize autofluorescent particles in the vascular wall of pial arteries from drug-resistant epilepsy patients who underwent resective brain surgery. Based on their (auto)fluorescent properties, these particles were characterized as lipofuscincontaining particles. The observation of these particles in the vascular wall was a finding that had not been reported on in literature to date.

Lipofuscin is formed during oxidative stress ${ }^{70,71}$. Oxidative stress applies to a condition of high metabolic activity, in which there is excessive generation of reactive oxygen species (ROS) that cannot be scavenged by the antioxidant defense system, resulting in structural cell damage ${ }^{72-74}$. Under physiological conditions, ROS are synthesized during aerobic metabolism. At high levels, ROS damage the cell by peroxidation ${ }^{74}$. Lipofuscin is a non-degradable substance that accumulates during ageing. Hence, lipofuscin may be a marker of the amount of endured oxidative stress.

In this respect, the observation of lipofuscin particles within the vascular wall of cerebral vessels may indicate oxidative stress within these vessels. This local oxidative stress marker may then be studied in relation to the pathophysiology of the underlying disease, i.e. drug-resistant epilepsy. Therefore, it was important to include cerebral vessels from non-epileptic controls. This brought us to the final research question of this thesis:

\section{Do lipofuscin particles in the vascular wall have different properties in epilepsy patients when compared to controls?}

For this study (chapter 6), cerebral arteries and brain tissue from non-epileptic patients was included as control tissue. Controls included post-mortem tissue of patients that were not suffering from a neurological disorder and tissue from a patient with a subcortical brain tumor that was obtained during surgery.

This learned that similar autofluorescent particles could be observed in cerebral vessels of these controls and that the autofluorescent spectrum of these particles was comparable. Yet, the number of lipofuscin particles was remarkably high in some epilepsy patients, suggesting an epilepsy-associated increase of lipofuscin. This increase remained when the age of the patients and controls was taken into account. This is of importance as lipofuscin accumulates with age.

An epilepsy-related increase of lipofuscin indicates high levels of oxidative stress in cerebral vascular cells of epilepsy patients. This suggestion might relate to the vascular 
abnormalities described above in combination with repetitive seizures that created extreme metabolic demands ${ }^{33}$. Indeed, the above-described microvascular alterations like increased BBB permeability, dysfunctional neurovascular coupling, and aberrant microvessels are all characterized by an increase of local and vascular oxidative stress $^{10,12,37,75-79}$. Moreover, increased oxidative stress and lipofuscin accumulation diminish the removal of newly generated cellular waste-material thereby further inducing cellular oxidative stress and contributing to cellular dysfunction ${ }^{71}$.

Our results should however be interpreted cautiously due to the low sample size, and potential confounders, such as another underlying disorder in controls. Nevertheless, one could hypothesize that reducing oxidative stress interferes the downward spiral of high oxidative stress levels, lipofuscin formation and cellular dysfunction. Since an intact glycocalyx has a high capacity to diminish activity of oxygen radicals by scavenging enzymes, like extracellular superoxide dismutase, it reduces oxidative stress and prevents endothelial dysfunction ${ }^{1,80}$. Hence, preservation of the glycocalyx could perhaps reduce vascular oxidative stress and subsequent vascular dysfunction. Conversely, a disrupted glycocalyx could increase the deleterious effects of oxidative stress and may result in lipofuscin formation in vascular cells.

\section{Conclusions and future perspectives}

This thesis explored the role of three vascular topics in relation to cerebrovascular physiology and the pathophysiology of epilepsy. The aim of the described experiments was to find novel targets for antiepileptic therapy. This general discussion therefore concludes by evaluating our results in this regard.

Firstly, the cerebrovascular glycocalyx may be an interesting novel target that can be modulated. As the most luminal part of the cerebral microvascular endothelium, it has an important barrier function, plays a role in endothelial vasomotor control, and reduces oxidative stress. In this thesis, we have shown that the cerebral microcirculation indeed includes a glycocalyx and hypothesized on its role in endothelial functioning. As the clinical case-control study is still ongoing and cerebrovascular glycocalyx properties of TLE-patients and non-epileptic controls are not compared in this thesis, no firm conclusions can be made regarding glycocalyx 'health' in TLE-patients. Upon closure of inclusion of the case - control study, the results will be evaluated in relation to epilepsy-specific characteristics, hemodynamic parameters, histopathological outcome, and the influence of possible confounders.

When future results show that the cerebral microcirculation of TLE-patients is characterized by a disrupted glycocalyx, restoration and preservation of the glycocalyx may be a potential new therapeutic target. In case of epilepsy-related glycocalyx disruption, preclinical and clinical studies should be designed to assess whether glycocalyx preservation has antiepileptogenic effects, and glycocalyx restoration has antiepileptic effects. Moreover, studies addressing the barrier function of the 
glycocalyx, as a possible player in the BBB, could be designed. By example, glycocalyx properties could be related to biomarkers of BBB leakage or radiological BBB leakage characteristics.

If sublingual and cerebral measurements correlate, sublingual assessments can be used to predict cerebrovascular glycocalyx properties. It could then be interesting to assess the sublingual glycocalyx of patients presenting after a first seizure in order to find microvascular predictors to differentiate a single epileptic seizure from new-onset epilepsy. In addition, these measurements might contribute to early recognition of drug-resistant epilepsy leading to earlier and patient-tailored interventions.

More upstream, the regulation of pial artery vasoconstriction, important to adjust local cerebral blood flow, was found to require a synergistic neurogenic and adrenergic interaction that is mediated by ET-1. We could not compare the findings in pial arteries of drug-resistant epilepsy patients due to a lack of adequate non-epileptic controls. It would nevertheless be very interesting to assess if the observed vasoconstrictive properties of pial arteries are physiological or indicate vascular dysfunction due to epilepsy-related causes. In general, additional experiments, such as the inclusion of cotransmitters and experiments in the absence of endothelium, are required to further elucidate the physiological mechanisms of cerebral artery vasoconstriction. This could reveal how NA modulates ET-1. A preclinical epilepsy model that allows comparison to a control group will reveal possible epilepsy-specific differences. Whether possible vasomotor control abnormalities constitute a novel therapeutic target for epilepsy is currently indistinct.

In similar pial arteries, we have demonstrated the abundant presence of lipofuscin in the artery wall of drug-resistant epilepsy patients. Based on these results, we elaborated that lipofuscin may be a biomarker for oxidative stress within the cells of the vascular wall. Hence, the finding of lipofuscin in the cerebral artery wall rather seems an epiphenomenon of vascular dysfunction than a pathophysiological hallmark on itself. As such, lipofuscin itself does not seem an interesting novel target for antiepileptic therapy. Nevertheless, lipofuscin as a biomarker for accumulative oxidative stress is a very interesting finding and should further be explored, especially in the light of epilepsy-related cognitive deterioration, accelerated ageing and the concept of epilepsy being a neurodegenerative disorder. Moreover, future studies on the formation of lipofuscin in epilepsy patients and healthy, variably aged, controls will contribute to a better understanding of oxidative stress and vascular dysfunction in epilepsy. 


\section{References}

1. Reitsma S, Slaaf DW, Vink H, Van Zandvoort MAMJ, Oude Egbrink MGA. The endothelial glycocalyx: Composition, functions, and visualization. Pflugers Arch Eur J Physiol. 2007;454:345-59.

2. Weinbaum S, Tarbell JM, Damiano ER. The structure and function of the endothelial glycocalyx layer. Annu Rev Biomed Eng. 2007;9:121-67.

3. Dane MJC, van den Berg BM, Lee DH, et al. A microscopic view on the renal endothelial glycocalyx. Am J Physiol Renal Physiol. 2015;ajprenal.00532.2014.

4. Ebong EE, Lopez-Quintero S V, Rizzo V, Spray DC, Tarbell JM. Shear-induced endothelial NOS activation and remodeling via heparan sulfate, glypican-1, and syndecan-1. Integr Biol. (Camb). 2014;6:338-47.

5. Pries a R, Secomb TW, Gaehtgens P. The endothelial surface layer. Pflugers Arch. 2000;440:65366.

6. Constantinescu AA, Vink H, Spaan JE. Endothelial cell glycocalyx modulates immobilization of leukocytes at the endothelial surface. Arterioscler. Thromb Vasc Biol. 2003;23:15417.

7. Rops AL, van den Hoven MJ, Baselmans MM, et al. Heparan sulfate domains on cultured activated glomerular endothelial cells mediate leukocyte trafficking. Kidney Int. 2008;73:52-62.

8. Florian JA, Kosky JR, Ainslie K, Pang Z, Dull RO, Tarbell JM. Heparan sulfate proteoglycan is a mechanosensor on endothelial cells. Circ Res. 2003;93:e136-42.

9. Abbott NJ, Patabendige AAK, Dolman DEM, Yusof SR, Begley DJ. Structure and function of the bloodbrain barrier. Neurobiol Dis.; 2010;37:13-25.

10. Heinemann U, Kaufer D, Friedman A. Blood Brain Barrier Dysfunction, TGFb signaling and AStrocyre Dysfunction in Epilepsy. Glia. 2012;60:1251-7.

11. van Vliet EA, Aronica E, Gorter JA. Role of blood-brain barrier in temporal lobe epilepsy and pharmacoresistance. Neuroscience 2014;277:455-73.

12. Rigau V, Morin M, Rousset $M-C$, et al. Angiogenesis is associated with blood-brain barrier permeability in temporal lobe epilepsy. Brain. 2007;130:1942-56.

13. Michalak Z, Lebrun A, Di Miceli M, et al. IgG leakage may contribute to neuronal dysfunction in drugrefractory epilepsies with blood-brain barrier disruption. J Neuropathol Exp Neurol. 2012;71:826-38.

14. Heinemann U, Kaufer D, Friedman A. Blood-brain barrier dysfunction, TGF $\beta$ signaling, and astrocyte dysfunction in epilepsy. Glia. 2012:1251-7.

15. Vezzani A, Friedman A, Dingledine RJ. The role of inflammation in epileptogenesis. Neuropharmacology. 2013:16-24.

16. Fabene PF, Laudanna C, Constantin G. Leukocyte trafficking mechanisms in epilepsy. Mol Immunol. 2013;55:100-4.

17. Alphonsus CS, Rodseth RN. The endothelial glycocalyx: A review of the vascular barrier. Anaesthesia. 2014;69:777-84.

18. Dane MJC, Van Den Berg BM, Avramut MC, et al. Glomerular endothelial surface layer acts as a barrier against albumin filtration. Am J Pathol. 2013;182:1532-40.

19. Neuhaus W, Germann B, Plattner VE, Gabor F, Wirth M, Noe CR. Alteration of the glycocalyx of two blood-brain barrier mimicking cell lines is inducible by glioma conditioned media. Brain Res. 2009;1279:82-9.

20. Mazzetti S, Librizzi L, Frigerio S, de Curtis M, Vitellaro-Zuccarello L. Molecular anatomy of the cerebral microvessels in the isolated guinea-pig brain. Brain Res. 2004;999:81-90.

21. Zhu J, Li X, Yin J, Hu Y, Gu Y, Pan S. Glycocalyx degradation leads to blood-brain barrier dysfunction and brain edema after asphyxia cardiac arrest in rats. J Cereb Blood Flow Metab. 2017;0271678X1772606.

22. Thom M. Review: Hippocampal sclerosis in epilepsy: A neuropathology review. Neuropathol Appl Neurobiol. 2014:520-43.

23. Pitkänen A, Lukasiuk K. Molecular and cellular basis of epileptogenesis in symptomatic epilepsy. Epilepsy Behav. 2009;14:16-25.

24. Kastanauskaite A, Alonso-Nanclares L, Blazquez-Llorca L, Pastor J, Sola RG, DeFelipe J. Alterations of the microvascular network in sclerotic hippocampi from patients with epilepsy. J Neuropathol Exp Neurol. 2009;68:939-50.

25. Morin-Brureau M, Lebrun A, Rousset $M-C$, et al. Epileptiform activity induces vascular remodeling and zonula occludens 1 downregulation in organotypic hippocampal cultures: role of VEGF signaling pathways. J Neurosci. 2011;31:10677-88. 
26. Pérez-Bárcena J, Romay E, Llompart-Pou JA, et al. Direct observation during surgery shows preservation of cerebral microcirculation in patients with traumatic brain injury. J Neurol Sci. 2015;353:38-43.

27. Pérez-Bárcena J, Goedhart P, Ibáñez J, et al. Direct observation of human microcirculation during decompressive craniectomy after stroke. Crit Care Med. 2011;39:1126-9.

28. Šitina M, Turek Z, Pařzková R, Černý V. In situ assessment of the brain microcirculation in mechanicallyventilated rabbits using sidestream dark-field (SDF) imaging. Physiol Res. 2011;60:75-81.

29. Wan Z, Ristagno G, Sun S, Li Y, Weil MH, Tang W. Preserved cerebral microcirculation during cardiogenic shock. Crit Care Med. 2009;37:2333-7.

30. Brown WR, Thore CR. Review: Cerebral microvascular pathology in ageing and neurodegeneration. Neuropathol App. Neurobiol. 2011;37:56-74.

31. Mott RT, Thore CR, Moody DM, Glazier SS, Ellis TL, Brown WR. Reduced ratio of afferent to total vascular density in mesial temporal sclerosis. J Neuropathol Exp Neurol. 2009;68:1147-54.

32. Alonso-Nanclares L, DeFelipe J. Alterations of the microvascular network in the sclerotic hippocampus of patients with temporal lobe epilepsy. Epilepsy Behav. 2014;38:48-52.

33. Zhao M, Nguyen J, Ma H, Nishimura N, Schaffer CB, Schwartz TH. Preictal and ictal neurovascular and metabolic coupling surrounding a seizure focus. J Neurosci. 2011;31:13292-300.

34. Winkler MKL, Chassidim Y, Lublinsky S, et al. Impaired neurovascular coupling to ictal epileptic activity and spreading depolarization in a patient with subarachnoid hemorrhage: Possible link to blood-brain barrier dysfunction. Epilepsia. 2012;53:22-30.

35. Muoio V, Persson PB, Sendeski MM. The neurovascular unit - concept review. Acta Physiol. 2014;210:790-8.

36. Jacob M, Chappell D, Becker BF. Regulation of blood flow and volume exchange across the microcirculation. Crit Care. 2016;1-13.

37. Parfenova H, Carratu P, Tcheranova D, Fedinec A, Pourcyrous M, Leffler CW. Epileptic seizures cause extended postictal cerebral vascular dysfunction that is prevented by HO-1 overexpression. AJP Hear Circ Physiol. 2005;288:H2843-50.

38. Lin C-L, Jeng AY, Howng S-L, Kwan A-L. Endothelin and subarachnoid hemorrhage-induced cerebral vasospasm: pathogenesis and treatment. Curr Med Chem. 2004;11:1779-91.

39. Compeer MG, Janssen GMJ, De Mey JGR. Endothelin-1 and endothelin-2 initiate and maintain contractile responses by different mechanisms in rat mesenteric and cerebral arteries. $\mathrm{Br} \mathrm{J}$ Pharmacol. 2013;170:1199-209.

40. Pagán RM, Martínez AC, Hernández M, et al. Endothelial and neural factors functionally involved in the modulation of noradrenergic vasoconstriction in healthy pig internal mammary artery. Biochem Pharmacol. 2012;83:882-92.

41. Nausch LWM, Bonev a. D, Heppner TJ, Tallini Y, Kotlikoff MI, Nelson MT. Sympathetic nerve stimulation induces local endothelial Ca2+ signals to oppose vasoconstriction of mouse mesenteric arteries. AJP Hear Circ Physiol. 2012;302:H594-602.

42. Hilgers RHP, De Mey JGR. Myoendothelial coupling in the mesenteric arterial bed; segmental differences and interplay between nitric oxide and endothelin-1. Br J Pharmacol. 2009;156:1239-47.

43. Van Riper DA, Bevan JA. Evidence that neuropeptide $Y$ and norepinephrine mediate electrical fieldstimulated vasoconstriction of rabbit middle cerebral artery. Circ Res. 1991;68:568-77.

44. Lee TJF, Chang $\mathrm{HH}$, Lee $\mathrm{HC}$, et al. Axo-axonal interaction in autonomic regulation of the cerebral circulation. Acta Physiol. 2011;203:25-35.

45. Bevan R, Dodge J, Nichols $P$, et al. Responsiveness of human infant cerebral arteries to sympathetic nerve stimulation and vasoactive agents. Pediatr Res. 1998;44:730-9.

46. Van Riper DA, Bevan JA. Selective variation of agonist and neurally mediated vasoconstriction with rabbit middle cerebral artery branch order. J Pharmacol Exp Ther. 1991;257:879-86.

47. Aldasoro M, Martínez C, Vila JM, Medina P, Lluch S. Influence of endothelial nitric oxide on adrenergic contractile responses of human cerebral arteries. J Cereb Blood Flow Metab. 1996;16:623-8.

48. Duckworth JW, Wellman GC, Walters CL, Bevan JA. Aminergic histofluorescence and contractile responses to transmural electrical field stimulation and norepinephrine of human middle cerebral arteries obtained promptly after death. Circ Res. 1989;65:316-24.

49. Thorin E, Nguyen TD, Bouthillier A. Control of vascular tone by endogenous endothelin-1 in human pial arteries. Stroke. 1998;29:175-80.

50. Iadecola C, Davisson RL. Hypertension and cerebrovascular dysfunction. Cell Metab. 2008;7(6):476-84. 
51. Devinsky O. Effects of Seizures on Autonomic and Cardiovascular Function. Epilepsy Curr. 2004;4:43-6.

52. Lok J, Gupta P, Guo S, et al. Cell-cell signaling in the neurovascular unit. Neurochem Res. 2007;32: 2032-45.

53. Longden TA, Nelson MT. Vascular Inward Rectifier K+ Channels as External K+ Sensors in the Control of Cerebral Blood Flow. Microcirculation. 2016;22:183-96.

54. Peterson EC, Wang Z, Britz G. Regulation of cerebral blood flow. Int J Vasc Med. 2011;2011.

55. Dora KA. Coordination of vasomotor responses by the endothelium. Circ. J. 2010;74:226-32.

56. Mccarron RM, Chen Y, Tomori T, et al. Endothelial-mediated regulation of cerebral microcirculation. J Physiol Pharmacol. 2006;57:133-44.

57. Grände G, Nilsson E, Edvinsson L. Comparison of responses to vasoactive drugs in human and rat cerebral arteries using myography and pressurized cerebral artery method. Cephalalgia 2013;33:152-9.

58. Bialecki RA, Fisher CS, Murdoch WW, Barthlow HG, Bertelsen DL. Functional comparison of endothelin receptors in human and rat pulmonary artery smooth muscle. Am J Physiol. 1997;272:L211-8.

59. Bevan RD, Dodge J, Nichols P, et al. Weakness of sympathetic neural control of human pial compared with superficial temporal arteries reflects low innervation density and poor sympathetic responsiveness. Stroke. 1998;29:212-21.

60. Handa Y, Caner H, Hayashi M, Tamamaki N, Nojyo Y. The distribution pattern of the sympathetic nerve fibers to the cerebral arterial system in rat as revealed by anterograde labeling with WGA-HRP. Exp Brain Res. 1990;82:493-8.

61. Cipolla MJ, Li R, Vitullo L. Perivascular innervation of penetrating brain parenchymal arterioles. J Cardiovasc Pharmacol. 2004;44:1-8.

62. Hama-Tomioka K, Kinoshita H, Azma T, et al. The role of 20-hydroxyeicosatetraenoic acid in cerebral arteriolar constriction and the inhibitory effect of propofol. Anesth Analg. 2009;109:1935-42.

63. Marin J, Recio L, Reviriego J, Sanchez-Ferrer CF, Salaices M. Effects of morphine on noradrenaline release from cerebral and peripheral vascular smooth muscle. Gen Pharmacol. 1986;17:705-8.

64. Biddle NL, Gelb AW, Hamilton JT. Propofol differentially attenuates the responses to exogenous and endogenous norepinephrine in the isolated rat femoral artery in vitro. Anesth Analg. 1995;80:793-9.

65. Seçkin $\mathrm{H}$, Yigitkanli $\mathrm{K}$, Besalti $\mathrm{O}$, et al. Lamotrigine attenuates cerebral vasospasm after experimental subarachnoid hemorrhage in rabbits. Surg Neurol. 2008;70:344-51.

66. Wang H, Gao J, Lassiter TF, et al. Levetiracetam is Neuroprotective in Murine Models of Closed Head Injury and Subarachnoid Hemorrhage. Neurocrit Care. 2006;5:85-92.

67. Chuang $\mathrm{Y}-\mathrm{C}$, Chuang $\mathrm{H}-\mathrm{Y}$, Lin T-K, et al. Effects of long-term antiepileptic drug monotherapy on vascular risk factors and atherosclerosis. Epilepsia 2012;53:120-8.

68. Ma H, Zhao M, Suh M, Schwartz TH. Hemodynamic Surrogates for Excitatory Membrane Potential Change During Interictal Epileptiform Events in Rat Neocortex. J Neurophysiol. 2008;101:2550-62.

69. Ma H, Zhao M, Schwartz TH. Dynamic neurovascular coupling and uncoupling during ictal onset, propagation, and termination revealed by simultaneous in vivo optical imaging of neural activity and local blood volume. Cereb Cortex. 2013;23(4):885-99.

70. Terman A, Brunk UT. Lipofuscin: mechanisms of formation and increase with age. APMIS. 1998;106:265-76.

71. Brunk UT, Terman A. The mitochondrial-lysosomal axis theory of aging: Accumulation of damaged mitochondria as a result of imperfect autophagocytosis. Eur J Biochem. 2002;269:1996--2002.

72. Lushchak VI. Free radicals, reactive oxygen species, oxidative stress and its classification. Chem Biol Interact. 2014;224:164-75.

73. Sies H. Oxidative stress: oxidants and antioxidants. Exp Physiol. 1997;82:291-5.

74. Czerska M, Mikołajewska K, Zieliński M, Gromadzińska J, Wąsowicz W. Today's oxidative stress markers. Med Pr. 2015;66:393-405.

75. Brown WR, Thore CR. Review: Cerebral microvascular pathology in aging and neurodegeneration. Neuropathol Appl Neurobiol. 2011;37:56-74.

76. Islam MT. Oxidative stress and mitochondrial dysfunction-linked neurodegenerative disorders. Neurol Res. 2017;39:73-82.

77. Marchi N, Lerner-Natoli M. Cerebrovascular remodeling and epilepsy. Neurosci. 2013;19:304-12.

78. van Vliet EA, Aronica E, Gorter JA. Blood-brain barrier dysfunction, seizures and epilepsy. Semin Cell Dev Biol. 2015;38:26-34. 
79. Iadecola C. Neurovascular regulation in the normal brain and in Alzheimer's disease. Nat Rev Neurosci. 2004;5:347-60.

80. Li Q, Bolli R, Qiu Y, Tang XL, Murphree SS, French B a. Gene therapy with extracellular superoxide dismutase attenuates myocardial stunning in conscious rabbits. Circulation. 1998;98:1438-48. 
Summary 



\section{Summary}

Epilepsy is one of the most common neurological disorders. The most frequent type of focal epilepsy is temporal lobe epilepsy (TLE). Despite of the availability of many antiepileptic drugs and surgical options, the socio-economic burden of drug-resistant epilepsy remains enormous. Treatment of these patients may be improved by drugs that target alternative parts of the underlying pathophysiologic mechanisms. After years of research centered around neuronal elements, the pathophysiology of epilepsy remains however not well understood. Recently, epilepsy research started focusing on the potential role of vascular dysfunction in epilepsy. An overview of this research is described in chapter 1 . This shows that vascular abnormalities, such as aberrant microvessels and blood-brain barrier (BBB) disruption, are common in TLE-patients. Inadequate neurovascular coupling and vascular oxidative stress have also been shown. This thesis describes research exploring neurovascular interactions by studying structural and functional properties of the human cerebral vasculature in relation to normal physiology of the brain and the pathophysiology of epilepsy.

\section{The cerebral microcirculation and cerebrovascular glycocalyx}

The first hypothesis explored in this thesis was that the cerebrovascular glycocalyx is disrupted in temporal lobe epilepsy patients. The glycocalyx is a gel-like layer lining the endothelium and is involved in many vascular functions including vascular permeability. As such, a disrupted glycocalyx would increase permeability of the cerebral microcirculation, as is seen in TLE. During resective epilepsy surgery performed at our department, the cerebral microcirculation is directly visible and thus available for evaluation. Hence, we aimed to develop a method that allows real-time intraoperative assessment of the cerebral microcirculation including its glycocalyx.

To this end, the first aim of this thesis was to review techniques that are currently used to assess the cerebrovascular glycocalyx (chapter 2). This literature study showed that the cerebrovascular glycocalyx has been studied sparsely and only in ex vivo conditions. To enable in vivo assessment of the glycocalyx, it was concluded that non-invasive videomicroscopy, such as sidestream darkfield (SDF)-imaging, is the most suitable technique because it does not require (toxic) fluorescent dyes. Yet, SDF-imaging does not allow qualitative evaluation of the glycocalyx and is prone to motion and pressure artefacts.

Next, the first hypothesis was further addressed by designing a case-control study. In this regard, we firstly described the protocol that was used to evaluate microvascular properties, including vessel density and glycocalyx thickness, of the sublingual, cortical, and hippocampal microcirculation in TLE-patients intraoperatively (chapter 3). The control group consisted of adult patients undergoing resective brain surgery for a tumor or a vascular abnormality, or burr hole surgery for tumor biopsy, since intracranial measurements in genuinely healthy humans were considered as unethical. 
The subsequent step addressing the first hypothesis was to assess the feasibility of applying SDF-imaging intraoperatively to evaluate the cerebral microcirculation and glycocalyx. The first findings are therefore reported in chapter 4 . This learned that SDFimaging was indeed useful to visualize the cerebral microcirculation and to assess several of its properties, such as vessel density and glycocalyx thickness, expressed as perfused boundary region (PBR), when adequate software was subjoined. Herewith, we demonstrated technical feasibility of the developed method. Interestingly, the vessel density in the cortex appeared lower than in sublingual and hippocampal tissue. Furthermore, the PBR in the cortical microcirculation was substantially higher than in the sublingual and hippocampal microcirculation, suggesting a more unstable, i.e. disrupted, glycocalyx. Both findings may reflect physiological variations between the microvasculature of the temporal neocortex and that of the hippocampus. Alternatively, they reflect a failure of the cortical microcirculation to compensate for epilepsy-induced metabolic changes, or vice versa reflect a compensating recruitment of the hippocampal microcirculation due to local pathophysiologic metabolic alterations in epilepsy. The data presented here should be interpreted cautiously due to the low number of patients that were included, the lack of controls, and the influence of other potential confounders.

Our case-control study was designed to address the first hypothesis. Since patient inclusion and measurements are still ongoing, this thesis cannot describe outcome of the completed study.

\section{Vasomotor control of cerebral arteries}

During seizures, the cerebrovascular bed is exposed to major hemodynamic alterations while local brain metabolic demands are highly variable. Adequate adaptation of local blood flow is crucial to limit subsequent neuronal injury. The processes preserving adequate cerebral blood flow within acceptable hemodynamic ranges are orchestrated by vasomotor control of cerebral arteries and arterioles. Vasomotor control is determined by several signalling mechanisms including neurogenic, endothelial, endocrine, and metabolic. The precise interactions between these mechanisms are however unknown. Recent literature suggests that some of these mechanisms are dysfunctional in epilepsy.

Classically, arterial vasoconstriction is described as the result of sympathetic nerve release of noradrenaline (NA). Recent literature also suggests a role for endothelin-1 (ET-1) in arterial vasoconstriction. Regarding vasoconstriction in cerebral arteries, the influence of sympathetic nerves and NA is unclear, whereas the role of ET-1 has only been studied sparsely. Hence, the second hypothesis of this thesis was that cerebral artery vasoconstriction is controlled by endothelin 1. To this end, we assessed vasoconstrictive responses of cerebral arteries to neurogenic sympathetic stimulation in the presence of adrenergic and ET-1 modulating substances (chapter 5). Cerebral 
arteries were derived from epilepsy patients and compared to middle cerebral arteries from naive rats.

We found that cerebral artery vasoconstriction required a synergistic neurogenic and adrenergic activation and that this is ET-1 dependent. Based on these results, we hypothesized that NA modulates the release of ET-1 from endothelial cells and/or perivascular sensory nerves. Upon release, $\mathrm{ET}-1$ binds to the $\mathrm{ET}_{\mathrm{A}}$-receptor on smooth muscle cells thereby inducing vasoconstriction. This emphasizes the importance of interactions of different vasomotor control mechanisms.

We also observed differences in the vasoconstrictive responses of cerebral arteries from epilepsy patients and the controls. Because these two groups differ in many ways, these differences are difficult to interpret. Additional experiments to assess whether (a part of) these differences are related to the underlying disorder, i.e. epilepsy, may be addressed by a preclinical, better controlled study.

\section{Vascular oxidative stress}

During two-photon laser scanning microscopy evaluation of epilepsy patients' cerebral arteries, we serendipitously observed autofluorescent particles in the vascular wall (chapter 6). Because of the abundance of these particles, they were presumed to signify epilepsy-associated vascular pathology. Subsequent experiments aimed at characterizing the nature of these particles by evaluating their fluorescent properties, revealed that these particles indicate lipofuscin. Lipofuscin is a non-degradable substance that is formed during oxidative stress. This lead us to suggest that lipofuscin may be a marker of lifetime exposure to oxidative stress. Hence, cerebral arteries from epilepsy patients are exposed to high levels of oxidative stress. The structural and functional microvascular abnormalities that are described in epilepsy are indeed all characterized by an increase of vascular oxidative stress. Future studies on lipofuscin in epilepsy patients and healthy controls would contribute to a better understanding of the relation between oxidative stress and vascular dysfunction.

\section{Conclusion}

The aim of this thesis was to explore the potential role of structural and functional properties of the cerebral microvasculature in the pathophysiology of TLE. We demonstrated that SDF-imaging is a suitable technique to assess various properties, such as the glycocalyx thickness, of the cerebral microcirculation in vivo. Moreover, we found that the regulation of vasoconstriction in cerebral arteries requires synergistic neurogenic and adrenergic crosstalk mediated by ET-1. We also demonstrated the presence of lipofuscin in cerebral arteries of epilepsy patients suggesting vascular oxidative stress. Further research is required to reveal the precise role of these vascular structures and functions in relation to the pathophysiology of epilepsy. 

Samenvatting 



\section{Samenvatting}

Epilepsie is een van de meeste voorkomende neurologische aandoeningen. Temporaalkwab epilepsie (TLE) is de meest frequent voorkomende vorm van focale epilepsie. Ondanks dat er verschillende medicamenteuze en chirurgische therapeutische opties beschikbaar zijn, blijkt epilepsie in veel gevallen therapieresistent met enorme socio-economische en maatschappelijke gevolgen. Het is daarom van belang om nieuwe behandelingsmogelijkheden te ontwikkelen die aangrijpen op andere delen van de onderliggende pathofysiologie. De pathofysiologie van epilepsie is echter nog niet volledig ontrafeld ondanks vele jaren onderzoek dat zich met name heeft gericht op het functioneren van neuronen.

Epilepsieonderzoek richt zich sinds kort meer op vasculaire afwijkingen in epilepsie. In de algemene introductie (hoofdstuk 1 ) is een overzicht van deze onderzoeken gegeven. Hieruit blijkt dat TLE-patiënten vaak structurele vasculaire afwijkingen hebben zoals abnormale microvaten en disfunctioneren van de bloed-hersen barrière. Daarnaast vertonen deze vaten afwijkingen in de neurovasculaire koppelingsmechanismen en worden ze gekenmerkt door verhoogde oxidatieve stress. In dit proefschrift wordt exploratief onderzoek beschreven dat gericht is op neurovasculaire interacties door het bestuderen van structurele en functionele eigenschappen van de humane cerebrale vascularisatie in relatie tot zowel normale fysiologie als de pathofysiologie van epilepsie.

\section{De cerebrale microcirculatie en de cerebrovasculaire glycocalyx}

De eerste hypothese van dit proefschrift is dat de cerebrovasculaire glycocalyx verstoord is in patiënten met temporaalkwab epilepsie. De glycocalyx is een gelachtige bekleding van de binnenwand van het endotheel en is betrokken bij verschillende vasculaire functies zoals vaatwandpermeabiliteit. Als zodanig zou een verstoorde glycocalyx resulteren in een verhoogde permeabiliteit van de cerebrale microcirculatie. Een verhoogde permeabiliteit is beschreven in TLE. Op onze afdeling neurochirurgie voeren wij resectieve epilepsiechirurgie uit bij TLE-patiënten. Tijdens dergelijke operaties is de cerebrale microcirculatie direct zichtbaar en dus beschikbaar om in vivo te beoordelen. Om die reden hebben wij gepoogd om een methode te ontwikkelen waarmee we de cerebrale microcirculatie intra-operatief kunnen visualiseren en ook de glycocalyx kunnen beoordelen.

Het eerste doel van dit proefschrift was dan ook om alle momenteel beschikbare technieken om de cerebrovasculaire glycocalyx te beoordelen te evalueren (hoofdstuk 2). Deze literatuurstudie maakte duidelijk dat deze glycocalyx zelden is onderzocht en alleen in ex vivo omstandigheden. Om de cerebrovasculaire glycocalyx in vivo te beoordelen, werd videomicroscopie, zoals de sidestream darkfield (SDF) visualisatietechniek, als de meest geschikte techniek beschouwd omdat deze techniek niet-invasief is en geen (toxische) fluorescentie kleuroplossingen vereist. Een 
kwalitatieve analyse van de glycocalyx is echter niet mogelijk met deze techniek, die bovendien gevoelig is voor bewegings- en drukartefacten.

Daarna werd de eerste hypothese verder onderzocht door een case-control studie te ontwikkelen. Hoofdstuk $\mathbf{3}$ beschrijft het protocol van de studie waarin we beogen de intra-operatieve beoordeling van microvasculaire eigenschappen, zoals vaatdichtheid en glycocalyxdikte, van de sublinguale, corticale en hippocampale microcirculatie in TLE-patiënt mogelijk te maken. De controlegroep bestaat uit volwassen patiënten die resectieve hersenchirurgie ondergaan vanwege oncologische of vasculaire indicaties, of een boorgat operatie ten behoeve van tumorbioptie. Het is ethisch onaanvaardbaar invasieve intracraniële metingen te verrichten bij gezonde controles.

De volgende stap om de eerste hypothese te onderzoeken is het beoordelen van de technische uitvoerbaarheid om met SDF-techniek intraoperatieve metingen aan de cerebrale microcirculatie en glycocalyx te verrichten. De eerste bevindingen van onze metingen zijn daarom beschreven in hoofdstuk 4. Hieruit blijkt dat de SDF-techniek inderdaad geschikt is om de cerebrale microcirculatie te visualiseren. Met behulp van aanvullende software kunnen ook eigenschappen als vaatdichtheid en de glycocalyx, uitgedrukt als perfused boundary region (PBR), worden beoordeeld. Daarmee werd de technische uitvoerbaarheid aangetoond. Opvallend was dat de vaatdichtheid in de cortex lager was dan in sublinguaal en hippocampaal weefsel. Bovendien was de PBR van de corticale microcirculatie evident hoger dan in de sublinguale en hippocampale microcirculatie, hetgeen een instabielere, verstoorde, glycocalyx suggereert. Beide bevindingen kunnen berusten op fysiologische variatie van de verschillende lokale microcirculaties. Het kan ook een indicatie zijn dat de corticale microcirculatie onvoldoende kan aanpassen aan de lokale epilepsie-geinduceerde metabole vraag of juist dat de hippocampale microcirculatie sterk heeft gecompenseerd vanwege pathofysiologische metabole veranderingen gerelateerd aan epilepsie. Deze preliminaire resultaten moeten voorzichtig worden geïnterpreteerd gezien het lage aantal geïncludeerde patiënten, het ontbreken van controlepatiënten, en de invloed van verschillende confounders.

Het doel van de case-control studie is om de eerste hypothese te beantwoorden. Omdat de patiënten inclusie van de studie nog in volle gang is, beschrijft dit proefschrift niet de uitkomsten van de afgeronde studie.

\section{Vasomotore controle van cerebrale arteriën}

Tijdens epileptische insulten wordt de cerebrale vasculatuur blootgesteld aan grote hemodynamische veranderingen waarbij gelijktijdig de metabole vraag in verschillende hersengebieden sterk varieert. Het nauwkeurig aanpassen van de lokale bloedstroom is van groot belang om weefselschade te voorkomen. De processen om een voldoende cerebrale bloedstroom binnen acceptabele hemodynamische waarden te bewerkstelligen worden afgestemd door middel van vasomotore controle. Vasomotore controle wordt bepaald op basis van door neurogene, endotheliale, endocriene en 
metabole signalen. De exacte interacties tussen deze mechanismes is echter nog niet volledig bekend. Recente literatuur suggereert dat sommige van deze mechanismen verstoord zijn in epilepsie.

Klassiek gezien vindt arteriële vasoconstrictie plaats als het gevolg van release van noradrenaline (NA) uit sympathische zenuwen. Daarbij geeft recente literatuur aan dat endotheline-1 (ET-1) een belangrijke speelt bij deze vasoconstrictie. In cerebrale arteriën is de literatuur over het vasoconsrictieve effect van sympathetische zenuwen en NA niet eenduidig en is de rol van ET-1 hierin bovendien amper onderzocht. De tweede hypothese van dit proefschrift is dan ook dat vasoconstrictie van cerebrale arteriën wordt gereguleerd door endotheline-1. Daarom hebben we de vasconstrictieve respons van cerebrale arteriën op neurogene sympathische stimulatie beoordeeld in aan- en afwezigheid van verschillende adrenerge en ET-1 modulerende stoffen (hoofdstuk 5). Cerebrale arteriën waren afkomstig van epilepsiepatiënten en werden vergeleken met die van controle ratten.

Uit onze resultaten bleek dat vasoconstrictie van cerebrale arteriën plaatsvindt door een synergistisch effect van zowel neurogene en adrenerge activatie die ET-1 afhankelijk is. Op basis hiervan vormden wij de hypothese dat NA de afgifte van ET-1 moduleert, waarbij ET-1 wordt afgegeven door endotheelcellen en/of perivasculaire neuronen. Het vrijgekomen ET-1 bindt vervolgens aan de $\mathrm{ET}_{\mathrm{A}^{-}}$-receptor op vasculaire gladde spiercellen wat tot arteriële vasoconstrictie leidt. Onze resultaten benadrukken het belang van synergisme en interacties van de verschillende vasomotor controlemechanismes.

Verder laten onze vasoconstrictieve respons resultaten enkele verschillen zien tussen cerebrale arteriën van epilepsiepatiënten en gezonde ratten. Omdat er veel verschillen zijn tussen deze twee groepen is vergelijken lastig. Aanvullende experimenten om te beoordelen of (een deel) van de gevonden verschillen het gevolg zijn van de onderliggende epilepsie, kan in een preklinische studie met vergelijkbare controles worden onderzocht.

\section{Vasculaire oxidatieve stress}

Tijdens onderzoek met twee-fotonen laserscanning microscopie vielen autofluorescente partikels in de vaatwand van cerebrale arteriën van epilepsiepatiënten op (chapter 6). Door de enorme hoeveelheid van deze partikels, ontstond het vermoeden dat ze gerelateerd kunnen zijn aan epilepsie-geassocieerde vasculaire afwijkingen. Aanvullende experimenten om de partikels te karakteriseren op basis van hun fluorescentie eigenschappen toonden aan dat het om lipofuscine partikels ging. Lipofuscine is een niet-afbreekbaar eindproduct van oxidatieve stress. Lipofuscine zou dan ook een potentiële marker kunnen zijn voor de in een leven doorgemaakte oxidatieve stress. Dat suggereert dan ook dat cerebrale arteriën van epilepsie veel oxidatieve stress hebben doorgemaakt. Alle in epilepsie beschreven structurele en functionele microvasculaire afwijkingen hebben inderdaad een toename 
van oxidatieve stress tot gevolg. Toekomstige studies naar (vasculair) lipofuscine in epilepsie- en ook controlepatiënten kunnen bijdragen aan een beter begrip van de relaties tussen oxidatieve stress en vasculair disfunctioneren zoals zich voordoet in de pathofysiologie van epilepsie.

\section{Conclusie}

Het doel van dit proefschrift was om de rol van verschillende structurele en functionele eigenschappen van de cerebrale microcirculatie te verkennen in relatie tot de pathofysiologie van TLE. We hebben in dit proefschrift aangetoond dat videomicroscopie met SDF-techniek een geschikte techniek is om verschillende eigenschappen van de cerebrale microcirculatie, zoals glycocalyxdikte, te beoordelen. Ook hebben we aangetoond dat de regulatie van vasoconstrictie van cerebrale arteriën afhankelijk is van een synergistisch neurogeen en adrenerg effect dat resulteert in ET-1 gemedieerde contractie van gladde spiercellen. Bovendien hebben in dezelfde cerebrale arteriën van epilepsiepatiënten de aanwezigheid van lipofuscine aangetoond, hetgeen vasculaire oxidatieve stress suggereert. Toekomstig onderzoek is nodig om de exacte rol van deze vasculaire structuren en functies in relatie tot de pathofysiologie van epilepsie bloot te leggen. 
Valorisation 



\section{Valorisation}

\section{Relevance}

Epilepsy is one of the most common neurological disorders. The incidence and prevalence of epilepsy in developed countries, like the Netherlands, has recently been reported to be 25-50 per 100,000 people and 4-10 per 1,000 people, respectively ${ }^{1,2}$. Based on these numbers, it is estimated that there are around 100,000 patients with epilepsy in the Netherlands. Of these patients, $20-40 \%$ suffer from drug-resistant epilepsy ${ }^{2,3}$. Healthcare, societal and individual costs and burden related to epilepsy are relatively high due to the many diverse consequences of epilepsy. Chronic drugresistant epilepsy encompasses around $80 \%$ of the total epilepsy-related costs ${ }^{1,4}$.

Healthcare costs includes direct costs like regular follow-up at a neurologist and the use of anti-epileptic drugs. In addition, the costs related to seizures like injuries following a seizure and emergency care visits due to a status epilepticus should not be underestimated. Moreover, epilepsy patients frequently undergo an electroencephalogram and costly brain imaging to gain more information about the source of their seizures. Epilepsy surgery can be an alternative treatment for patients whom are selected by an extensive diagnostic workup.

Societal costs and burden related to epilepsy are remarkably high too. This is related to epilepsy-associated alterations in physical, psychological and social functioning. Prevalence of psychologic morbidity, such as cognitive decline, depression and anxiety is high in epilepsy patients ${ }^{5}$. Societal participation of adult epilepsy patients is reduced due to low employment rates and, when employed, high rates of sick leave (6). The addition of driver's license restrictions further complicates participation in our society for epilepsy patients. Not surprisingly, self-reported quality of life is low in these patients ${ }^{5}$.

Despite of many years of basic and clinical research, the pathophysiology of epilepsy has not been fully elucidated to date. For drug-resistant epilepsy patients, currently available treatments do not offer a cure for their disorder or cause too severe adverse effects. These patients are awaiting new treatment options that may be aimed at yet unraveled mechanisms. The recent paradigm shift in epilepsy research towards the interactions between neuronal and vascular cells may generate novel targets for antiepileptic therapies. Since this thesis aimed to explore structures and functions of the neurovascular interface, the additional value of this thesis lies in the potential of finding novel targets for antiepileptic therapies.

\section{Potential targets described in this thesis}

This thesis aimed to increase our understanding of the interactions taking place at the human neurovascular interface in physiological conditions and in epilepsy. The scientific interest in neurovascular interactions in epilepsy research is the result of recently described neurovascular dysfunction in other neurological disorders, like stroke, 
Alzheimer's disease, cerebral small vessel disease and traumatic brain injury. Hence, the results of the experiments described in this thesis may also contribute to a better understanding of these neurological disorders.

The first potential target explored in this thesis was the cerebrovascular glycocalyx. We started off by hypothesizing on the role of this glycocalyx as part of the cerebral microvascular endothelium. Considering this role as such, a disrupted glycocalyx may result in increased vascular permeability, dysregulated vasomotor control and/or vascular oxidative stress. Epilepsy and the other above-mentioned neurological disorders are characterized by one or more of these vascular abnormalities, making the cerebrovascular glycocalyx a scientifically, and perhaps therapeutically, interesting part of the cerebral microcirculation.

The regulation of cerebral blood flow by means of vasomotor control has also been explored in this thesis. Chapter 5 focused on neurogenic and adrenergic crosstalk in pial artery vasoconstriction in epilepsy patients. It was found that endothelin-1 plays an eminent role in this crosstalk. Due to the lack of adequate non-epileptic controls, we could not distinguish whether differences in vasoconstrictive responses noted in cerebral arteries of epilepsy patients are attributable to epilepsy or to the many confounding variables. Hence, it remains indistinct whether possible vasomotor control abnormalities constitute a novel therapeutic target for epilepsy.

In similar pial arteries, abundant numbers of lipofuscin particles were demonstrated in cerebral arteries from drug-resistant epilepsy patients. Based on literature, lipofuscin was suggested as a biomarker for oxidative stress. Consequently, it was suggested that vascular cells of cerebral arteries in epilepsy patients are exposed to multiple periods of oxidative stress. This could perhaps be the results of repetitive seizures in these drugresistant epilepsy patients. Lipofuscin in the cerebral artery wall seems an epiphenomenon of vascular dysfunction rather than a pathophysiological hallmark on itself. Hence, lipofuscin itself does not seem an interesting novel target for antiepileptic therapy. Nevertheless, vascular oxidative stress might well contribute to or be the consequence of vascular dysfunction. As such, vascular oxidative stress as a therapeutic target in cerebral arteries of epilepsy patients remains to be further explored.

\section{Future perspectives}

The first future perspective lies in completing our case-control study in order to demonstrate possible alterations in the cerebral microcirculation, like glycocalyx properties, of epilepsy patients. Future results may show that the cerebral microcirculation of these patients is characterized by a disrupted glycocalyx, as hypothesized in this thesis. In that case, glycocalyx preservation may have antiepileptogenic effects, and restoration of the glycocalyx might have antiepileptic effects. Studies addressing the barrier function of the glycocalyx, as a possible player in the blood-brain barrier (BBB), could also be considered. For example, glycocalyx properties could be related to biomarkers of BBB leakage, or may on itself function as a 
biomarker for BBB leakage. Glycocalyx properties could also be related to the radiological demonstration of $B B B$ leakage.

Moreover, when sublingual measurements performed in epilepsy patients predict cerebral microvascular abnormalities, it is of great interest to assess the sublingual microcirculation in patients after their first seizure. Sublingual microvascular properties may then be used to differentiate a single epileptic seizure from new-onset epilepsy and may lead to early recognition of drug-resistant epilepsy resulting in patient-tailored therapy.

In this thesis, we describe preliminary results of in vivo measurements of the glycocalyx of cerebral microvessels of epilepsy patients. We were also able to analyze other microvascular properties such as vessel density. The technique described in this thesis may therefore significantly contribute to research on the role of microvascular pathophysiology in other neurological disorders.

Future studies should also focus on the question whether vasomotor control is abnormal in epilepsy and other neurological disorders. In this regard, a preclinical casecontrol studies could be designed. Restoration of adequate vasomotor control might subsequently constitute a novel therapeutic target for epilepsy.

Finally, lipofuscin as a biomarker for accumulative oxidative stress is an interesting future study topic. These studies can focus on the formation of lipofuscin in epilepsy patients and healthy, variably aged, controls in order to contribute to a better understanding of oxidative stress and vascular dysfunction in epilepsy. Such studies could subsequently result in novel targets for antiepileptic therapies.

As mentioned, the results of this thesis hopefully also fuel new research on other neurological disorders that are characterized by neurovascular dysfunction like stroke, Alzheimer's disease, cerebral small vessel disease and traumatic brain injury. 


\section{References}

1. Engel J, McDermott MP, Wiebe S, Langfitt JT, Stern JM, Dewar S, et al. Early surgical therapy for drugresistant temporal lobe epilepsy: a randomized trial. JAMA. 2012;307(9):922-930.

2. Téllez-Zenteno JF, Hernández-Ronquillo L. A review of the epidemiology of temporal lobe epilepsy. Epilepsy Res Treat. 2012;2012:630853.

3. Kwan P, Arzimanoglou A, Berg AT, Brodie MJ, Allen Hauser W, Mathern G, et al. Definition of drug resistant epilepsy: consensus proposal by the ad hoc Task Force of the ILAE Commission on Therapeutic Strategies. Epilepsia 2010;51(6):1069-1077.

4. Pierzchala K. Pharmacoresistant epilepsy - epidemiology and current studies. Neurol Neurochir Pol. 2010;44(3):285-290.

5. Taft C, Sager Magnusson E, Ekstedt G, Malmgren K. Health-related quality of life, mood, and patient satisfaction after epilepsy surgery in Sweden--a prospective controlled observational study. Epilepsia 2014;55(6):878-885.

6. Allers K, Essue BM, Hackett ML, Muhunthan J, Anderson CS, Pickles K, et al. The economic impact of epilepsy: a systematic review. BMC Neurol. 2015;15:245. 
Dankwoord 



\section{Dankwoord}

Een proefschrift schrijven doe je niet alleen. Zonder de steun, tijd en vele uren werk van anderen zou dit proefschrift niet tot stand zijn gekomen. Ik realiseer mij hoe bijzonder dit is en ik ben daar erg dankbaar voor. Een aantal personen wil ik in het bijzonder bedanken.

Prof. dr. Y. Temel, beste Yasin, graag wil ik jou bedanken voor het vertrouwen dat je mij en de co-promotores in de eindfase van dit proefschrift hebt gegeven. In onze dagelijkse samenwerking in de kliniek, op OK maar ook bij onze sectie-werkzaamheden, ervaar ik hetzelfde vertrouwen. Jouw visie en oneindige motivatie om ons vakgebied vooruit te helpen, zijn een enorme inspiratie voor mij. Bedankt dat je mijn promotor wilt zijn.

Prof. dr. J.J. van Overbeeke, beste Koo, bedankt dat jij zowel mijn opleiding als promotieonderzoek mogelijk hebt gemaakt. Dit proefschrift was er niet geweest zonder jouw steun. Helaas is de laatste fase anders gelopen dan we hadden gedacht.

Dr. K. Rijkers, beste Kim, jij was degene die mij op sleeptouw nam toen ik voor de eerste keer bij de neurochirurgie in Maastricht kwam meekijken. Al snel was er een klik. Een klik die we nog steeds hebben. Je hebt mij zowel klinisch als wetenschappelijk enorm geholpen binnen de neurochirurgie. Dit proefschrift is voor een groot deel te danken aan jouw creativiteit. Op de belangrijkste momenten stond jij op en kwam jij met een plan. Bedankt voor al je hulp, oprechtheid, enthousiasme, tijd, en slimheid! Je bent op alle fronten een voorbeeld voor mij!

Dr. G. Hoogland, beste Govert, jij hebt mij wegwijs gemaakt in het lab en mij de kneepjes van basaal onderzoek geleerd. Toen we uiteindelijk een klinisch pad insloegen, waren jouw kritische blik en kennis eens te meer van grote waarde. Jouw praktische insteek (denken in grafieken en figuren) gaven mij steeds weer een duidelijk kader. Ik bewonder je rust, geduld en bescheidenheid. Bedankt voor al je hulp en tijd!

Prof. dr. H. Vink, beste Hans, direct na onze eerste ontmoeting heb ik Kim en Govert gemaild dat we iets met 'jouw' glycocalyx moesten doen. Jouw overtuigingskracht en enthousiasme bewonder ik enorm. Zonder jouw introductie in de wereld van de microcirculatie en glycocalyx was de glycolepsie-studie er nooit geweest. Bedankt voor je vertrouwen, de wijze lessen en het geduld dat je hebt gehad met mij en de vele studenten.

Prof. dr. M.A.M.J. van Zandvoort, beste Marc, jouw aanstekelijke enthousiasme, je toegankelijkheid, en vrolijkheid maken samenwerken met jou tot een feest. Bedankt voor je vertrouwen in mij, en ook in de studenten die ik steeds bij jou mocht afleveren. 
Je hebt mij veel geleerd over microscopie maar ook over de benadering van wetenschappelijke vraagstukken. Onze voorliefde voor dezelfde koffietent maakte de samenwerking compleet.

Prof. dr. J. de Mey, beste Jo, jij hebt mij op het pad van Endotheline-1 gezet. Een pad dat ik lange tijd als zeer complex heb beschouwd, en stiekem nog steeds beschouw. Dankzij jouw geduldige uitleg (altijd met een tekening) werd mij langzaam duidelijk wat we wel en vooral niet weten over elektrische veldstimulatie, vasoconstrictie en de diverse farmaca. Bedankt voor je pragmatische en realistische aanpak.

Dr. O.E.M.G. Schijns, dr. J. Dings, beste Olaf, en beste Jim, als epilepsie-neurochirurgen zijn jullie vanaf dag één bij dit onderzoek betrokken geweest. Bedankt voor de geboden mogelijkheden tijdens de operaties en jullie kritische blik gedurende het onderzoek. Hiervoor ben ik jullie erg dankbaar en ik hoop nog veel met jullie te mogen opereren.

Dr. P. Schiffers, beste Paul, je hebt ontzettend veel werk verzet om de farmacologische experimenten te laten slagen. Werk waarvan ik pas later echt begreep wat het inhield. Bedankt voor al je werk en het vele geduld dat je hebt gehad.

Dr. J. Staals, beste Julie, bedankt voor jouw neurovasculaire en kritische wetenschappelijke bijdrage aan de glycolepsie-studie.

Dr. S.M.J. van Kuijk, beste Sander, bedankt voor jouw belangrijke en pragmatische statistische bijdragen aan dit proefschrift.

De beoordelingscommissie bestaande uit prof. dr. R. van Oostenbrugge, prof. dr. W. Backes, prof. dr. P. Fabene, dr. S. Reitsma en dr. A. Colon wil ik bedanken voor de beoordeling van dit proefschrift. Daarnaast wil ik prof. dr. M. Majoie en dr. L. Ackermans bedanken voor het plaatsnemen in de corona.

Bedankt neurochirurgen van de afdelingen neurochirurgie Maastricht en Heerlen voor de neurochirurgische opleiding en jullie belangstelling in mijn onderzoek. Ik ben jullie ook dankbaar dat jullie het doen van onderzoek naast het werken op OK en in de kliniek mogelijk maken. Ik geniet iedere dag van mijn opleiding en ben trots dit bijzondere vak van jullie te leren!

Graag bedank ik ook mijn collega-assistenten door de jaren heen: Vincent, Toon, Youssef, Felix, Anouk, Tim, Lars, Suzanne, Remco, Jeroen, Karlijn, en Rick voor de ruimte die jullie mij hebben gegeven om dit proefschrift te realiseren. In een klein team moeten we vaak hard werken om de kliniek overeind te houden. Het is mede dankzij jullie flexibiliteit dat ik dit proefschrift heb kunnen afronden naast de opleiding. Daarnaast haal ik veel plezier uit ons intensieve contact, zowel tijdens het werk als daarbuiten. Laten we dit vooral zo voortzetten! 
Ook alle collega's van het secretariaat, verpleegkundigen en operatieassistenten van de afdelingen neurochirurgie in zowel Maastricht als Heerlen: bedankt! Een speciaal woord van dank voor Trudy Stefess, Nicole Palmen, Miranda Spauwen-Liebens en Marina Vandeberg-Loyens voor jullie administratieve en praktische hulp bij dit proefschrift.

Mijn grote dank gaat ook uit naar de vele studenten die mij hebben geholpen met verschillende onderdelen van dit proefschrift. In het bijzonder wil ik Steffi van de Ven, Karlijn Hakvoort, Sieuwke Hartmans, Louise Otto en Rick van Lanen bedanken voor hun werk en bijdrage. Zonder jullie inzet was dit proefschrift nog lang niet klaar geweest! Jullie enthousiasme en kritische vragen hebben bovendien een extra dimensie gegeven aan het onderzoek. Daarnaast maakte het mij altijd erg trots om jullie in je onderzoeksstage te zien uitgroeien tot ware onderzoekers.

Beste Tom, oftewel Henk Plots, ofwel zeergeleerde dr. Stolp. Wat ben ik blij dat jij de afgelopen jaren in Maastricht bent blijven wonen. Onze festivalbezoekjes, de filosofieklasjes (en de vele biertjes na afloop) en natuurlijk onze wandelingen, zijn slechts een greep uit de gave avonturen die ik met jou heb beleefd. Jouw levensvisie, je oprechte mening en je creatieve ingevingen zijn enorm motiverend en inspirerend. Het is een understatement als ik zeg dat jij me door dit onderzoek hebt gesleept door me ook een andere kant van het leven te laten zien. Bedankt dat je mijn paranimf wilt zijn.

Beste Felix, oftewel Starsky, mijn neurochirurgische wederhelft. Vanaf de dag dat we écht samen over de afdelingen in Maastricht vlogen, kwamen we erachter hoeveel we gemeen hebben. Vanaf die dag zijn we niet langer collega's, maar vrienden. In ons dagelijkse werk maken we veel momenten mee die niet zijn uit te leggen aan de buitenwereld. Dat zijn de momenten die ik altijd graag met jou deel. Jouw rust bewonder ik en jouw vragen moet ik niet altijd willen beantwoorden. Ik hoop dat er nog vele congressen, etentjes en wijntjes mogen volgen. Bedankt dat jij mijn paranimf wilt zijn.

Bedankt Festglede-baasjes! Onze avonturen tijdens en na de studententijd zijn hoogtepunten uit mijn leven. De vriendschappen met jullie hebben mij gevormd en maken het leven mooi. FESTGLEDE HOOG!!

Felix Sion, wat begon als tennismaatjes groeide uit tot een vriendschap die nog steeds erg belangrijk voor me is. Samen hebben we de meest donkere en kleurrijke dagen beleefd. Bedankt dat we nog steeds vrienden zijn en dat dit zo vanzelfsprekend is.

Lieve Peter-Paul, Mieke, Jorren en Laurien. Al ruim 13 jaar zijn jullie mijn 'schoonfamilie'. We hebben het altijd erg goed kunnen vinden en vakanties met jullie 
zijn steeds genieten. Ik beschouw jullie allang niet meer als de familie van Annemijn maar als mijn eigen familie. Bedankt voor jullie interesse en openheid.

Lieve pap en mam, Leo en Mechtild. Door jullie open, eerlijke, rechtvaardige, en sociale opvoeding, kan ik mijn werk goed relativeren en in een maatschappelijke context plaatsen. Jullie vormen voor mij de warme basis, en hebben mij altijd onvoorwaardelijk gesteund. Door jullie hulp, toewijding en vooral liefde heb ik bereikt wat ik zo graag wilde bereiken. Mijn dankbaarheid voor alles wat jullie voor mij gedaan hebben, is niet in woorden uit te drukken.

Lieve Geert en Marieke, mijn broer en zusje. We lijken in veel opzichten niet op elkaar, maar stiekem toch meer dan we denken. Door de jaren is er een mooie band tussen ons ontstaan en weet ik dat ik altijd op jullie kan terugvallen. Ik ben trots op wie jullie zijn, met wie (Maartje en Remo!) jullie het leven delen en waar jullie voor staan.

Lieve Annemijn, hoe kan ik jou in 1 alinea bedanken. Jij die mij altijd op alle fronten helpt en steunt. We zijn alweer 13 jaar samen. In die 13 jaar was ik altijd druk, steeds weer met een ander project. En altijd steunde jij mij daarin, sterker nog, je moedigde mij aan. Je kent mij beter dan ik mezelf ken. Doordat jij rust creëert op alle andere vlakken, en door jouw evenwichtigheid, kon ik dit proefschrift afronden. Ik hoop dat ik de rust kan vinden om na mijn verdediging toch echt meer tijd voor jou te maken, dat heb je verdiend. Bedankt dat je me laat zijn en doen. Bedankt dat je bestaat en bij mij wilt zijn. Ik hoop dat we nog vele jaren mogen samenzijn. 
Curriculum vitae 



\section{Curriculum vitae}

Roel Haeren werd op 14 december 1986 geboren in Heerlen. In 2005 slaagde hij cum laude voor het Gymnasium aan het Bernardinuscollege te Heerlen. Daarnaast werd hij in de tussenliggende jaren als hardloper op de middellange afstanden meermaals Limburgs en Nederlands kampioen.

Vervolgens is hij geneeskunde gaan studeren aan de Universiteit Maastricht. Deze studie heeft hij een jaar onderbroken om als bestuurslid extern en vice-voorzitter van studentenvereniging KoKo werkzaam te zijn. Na het behalen van de artsenbul in 2012 begon hij te werken als arts-assistent op de afdeling Neurochirurgie van Maastricht Universitair Medisch Centrum. Aldaar is hij vanaf 2013 in opleiding tot neurochirurg met als opleiders prof. dr. J.J. van Overbeeke, dr. H. van Santbrink en dr. M. ter Laak. Tijdens zijn opleiding ontving hij de prijs 'clinicus van het jaar 2015: arts-assistent neurowetenschappen' voor zijn betrokkenheid bij het coassistenten onderwijs. Hij is tevens secretaris van de sectie 'functionele neurochirurgie' van de Nederlandse Vereniging voor Neurochirurgie.

Naast zijn klinische werkzaamheden werkte hij van 2012 tot 2017 aan het onderzoek dat geleid heeft tot dit proefschrift aan de School for Mental Health and Neuroscience van het Maastricht Universitair Medisch Centrum onder supervisie van prof. dr. Y. Temel, prof. dr. J.J. van Overbeeke, dr. K. Rijkers en dr. G. Hoogland.

Daarnaast is hij beleidsmatig geïnteresseerd, in het bijzonder in doelmatigheid van zorg. In 2015 won hij de 'doelmatigheidsprijs'. Nadien werd hij landelijk ambassadeur van doelmatigheid van zorg en participeerde hij in het landelijke 'Bewustzijnsproject'. In deze rol was hij betrokken bij de ontwikkeling van een tweetal online courses en een eTool over kosten van zorg en projectmatig werken. Daarnaast rondde hij in 2016 succesvol een minor 'Economics in Healthcare' aan de faculteit gezondheidswetenschappen van de Universiteit Maastricht af.

Roel woont samen met Annemijn de Leeuw in Maastricht. In 2019 verwacht hij zijn opleiding tot neurochirurg af te ronden. 

List of publications 



\section{List of publications}

M. Fens, C. van Heugten, G. Beusmans, M. Limburg, R. Haeren, A. Kaemingk, J. Metsemakers, Not as transient: patients with transient ischaemic attack or minor stroke experience cognitive and communication problems; an exploratory study, European Journal of General Practice, 2012.

R. Haeren, J. Dings, M. Hoeberigs, R. Riedl, K. Rijkers, Posttraumatic skull hemangioma: Case report, Journal of Neurosurgery, 2012.

R. Haeren, M. Lammens, L. Jacobi, H. van Santbrink, O. Schijns. Growing lumbar juxtafacet cyst causing conus medullaris syndrome, Acta Neurochirurgica, 2013.

M. Broen, R. Haeren, Y. Temel, E. Hoff, O. Schijns, Hemiballism-hemidystonia after parietal lobe tumour resection, Journal of Clinical Neurology and Neurosurgery, 2014.

R. Haeren, M. Hendriks, M. Vlooswijk, L. Verdyck, V. van Kranen, L. Wagner, S. van Benthum, O. Schijns, Deterioration of dyslexia after non-dominant temporal lobectomy for drug-resistant epilepsy. A unique case and literature review, Epileptic Disorders, 2016.

R. Haeren, S. van de Ven, M. van Zandvoort, H. Vink, J. van Overbeeke, G. Hoogland, K. Rijkers, Assessment and imaging of the cerebrovascular glycocalyx, Current Neurovascular Research, 2016.

R. Haeren, H. Vink, G. Hoogland, O. Schijns, J. Dings, K. Rijkers, Bloed-hersenbarrière onderzoek en epilepsie: drie nieuwe thema's, Epilepsie, periodiek voor professionals, 2016.

R. Haeren, M. Hendriks, M. Vlooswijk, L. Verdyck, V. van Kranen, L. Wagner, S. van Benthum, O. Schijns, Verslechtering van pre-existente dyslexie na temporaalkwabresectie van de niet-dominante hemisfeer, Epilepsie, periodiek voor professionals, 2016.

R. Haeren, H. Vink, J. Staals, M. van Zandvoort, J. Dings, J. van Overbeeke, G. Hoogland, K. Rijkers, O. Schijns, Visualization and evaluation of the cerebrovascular glycocalyx in temporal lobe epilepsy: protocol of an observational study, BMJ Open, 2017.

R. Haeren, K. Rijkers, J. Staals, M. van Zandvoort, J. Dings, O. Schijns, J. van Overbeeke, G. Hoogland, H. Vink, Intraoperative assessment of the human cerebrovascular glycocalyx and microcirculation, Journal of Cerebrovascular Diseases, 2017. 
K. Hakvoort, R. Haeren, A. Smeets, H. van Santbrink, Extracraniële uitbreiding van een glioblastoom door een boorgat: presentatie als wondprobleem, Tijdschrift voor neurologie en neurochirurgie, 2017.

R. Haeren, S. Hartmans, G. Hoogland, J. Dings, O. Schijns, J. van Overbeeke, J. de Mey, S. van Kuijk, K. Rijkers, P. Schiffers, Cerebral artery vasoconstriction requires neurogenic and adrenergic interaction and is Endothelin-1 dependent, Current Neurovascular Research, 2017.

J. Habets, R. Haeren, S. Lie, N. Bauer, J. Dings, Acute monocular blindness due to orbital compartment syndrome following a pterional craniotomy: a case report', World Neurosurgery, 2018.

R. Haeren, K. Rijkers, G. Hoogland, O. Schijns, J. Dings, J. Staals, M. van Zandvoort, J. van Overbeeke, H. Vink, In vivo assessment of the human cerebral microcirculation and its glycocalyx: a technical report, Journal of Neuroscience Methods, 2018.

R. van Lanen, C. Hoeberigs, N. Bauer, R. Haeren, G. Hoogland, A. Colon, C. Piersma, J. Dings, O. Schijns, Quantification and analysis of visual field deficits following temporal lobectomy, Acta Neurochirurgica, 2018.

K. Hakvoort, L. Otto, R. Haeren, K. Rijkers, G. Hoogland, O. Schijns, J. Dings, J. van Overbeeke, M. van Zandvoort, Lipofuscin in cerebral arteries and tissue of epilepsy patients, submitted. 
